\title{
Mathematical Modelling of Acute Rheumatic Fever
}

Melissa Welsh

VICTORIA UNIVERSITY OF WELLINGTON

Te Whare Wananga o te Upoko o te Ika a Maui

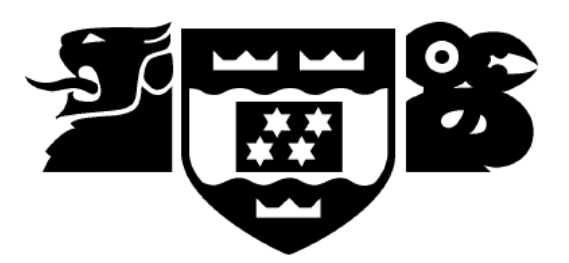

School of Mathematics, Statistics and Operations Research

Te Kura Mātai Tatauranga, Rangahau Pūnaha

\begin{abstract}
A thesis
submitted to the Victoria University of Wellington in fulfilment of the requirements for the degree of Master of Science in Mathematics.
\end{abstract}

Victoria University of Wellington

2012 


\begin{abstract}
Acute rheumatic fever is a major cause of heart disease in many parts of the world. Though it is generally considered rare in developed countries, is remains a large issue in New Zealand. Of particular concern is the prevalence of acute rheumatic fever among Maori and Pacific Island peoples. In this thesis we develop a model to simulate acute rheumatic fever in a population. We discuss the use of both deterministic methods and stochastic processes. Demographics and statistics specific to New Zealand are then used to develop the model in a way that fits specifically to the situation in New Zealand. We also consider the introduction of treatment strategies for acute rheumatic fever and discuss how risk factors can be used to focus such strategies.
\end{abstract}




\section{Acknowledgements}

I would like to thank Mark McGuinness and Nokuthaba Sibanda for their guidance, supervision and encouragement throughout the development of this thesis.

I would also like to thank Michael Welsh for his support and his help with using IATEX.

Thank you to my parents; Robert and May Dabb, for their support and encouragement and to Bruce Welsh, Esther Welsh and Matthew Bayliss for help with proof reading. 


\section{Contents}

$\begin{array}{llr}1 & \text { Introduction } & 1\end{array}$

2 Infectious Disease Modelling $\quad 3$

2.1 Compartmental Models . . . . . . . . . . . . . . . . . . 4

2.2 The basic reproduction number $\mathcal{R}_{0} \ldots \ldots \ldots \ldots$

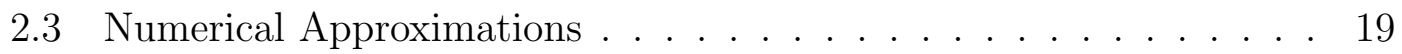

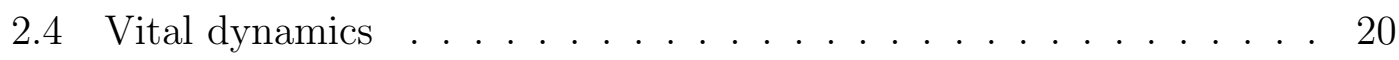

2.5 Stochastic Epidemic Models . . . . . . . . . . . . . . . . 24

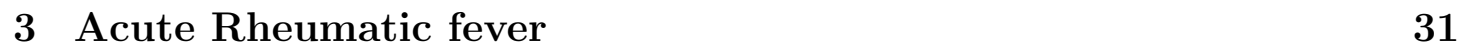

3.1 Symptoms and Diagnosis . . . . . . . . . . . . . . . . . . . 31

3.2 Susceptibility and Prevention $\ldots \ldots \ldots$. . . . . . . . . . . . 32

3.3 Treatment and Recovery . . . . . . . . . . . . . . . . . . . . . 34

3.4 Group A Streptococcus . . . . . . . . . . . . . . . . . . . . 34

3.5 New Zealand Infection Rates . . . . . . . . . . . . . . . . . . . . . . 37

4 A Basic Model for Rheumatic Fever 41

$4.1 \quad$ Building the Basic Model . . . . . . . . . . . . . . . . . . . . . 41

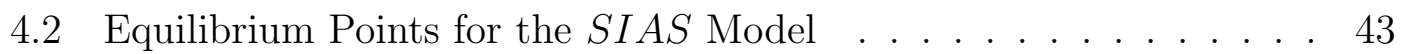

4.3 Triangle of Validity . . . . . . . . . . . . . . . . . . . . . . . . . . 47 
4.4 A Basic Stochastic Model . . . . . . . . . . . . . . . . . . . . . . . . 49

5 Developing the Model Further $\quad 54$

5.1 Vital Dynamics . . . . . . . . . . . . . . . . . . 54

5.2 Stochastic Model with Vital Dynamics . . . . . . . . . . . . . . . . 57

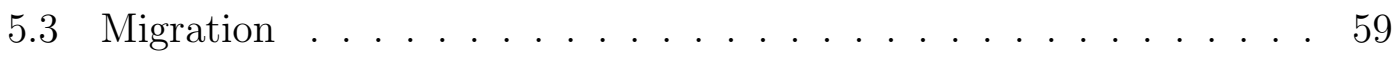

5.4 Exposed Periods and Treatment Models . . . . . . . . . . . . . . . 63

5.5 Acute Rheumatic Fever History . . . . . . . . . . . . . . . . . . . . 68

$\begin{array}{lll}6 & \text { A New Zealand Specific Model } & 75\end{array}$

6.1 Public Health records and treatment . . . . . . . . . . . . . . 76

6.2 Including Demographics $\ldots \ldots \ldots$. . . . . . . . . . . 86

6.3 Using a recommended diagnosis and treatment algorithm to define risk groups . . . . . . . . . . . . . . . . . . . . . . 93

\begin{tabular}{lll}
\hline 7 & Introducing Real Data and Conclusions & 103
\end{tabular}

7.1 Ethnic Proportions . . . . . . . . . . . . . . . . . . . . 104

7.2 Rates of Rheumatic Fever Development . . . . . . . . . . . . . . . . 104

7.3 Rates of Treatment and Recovery . . . . . . . . . . . . . . . . 105

7.4 Rates of Infection . . . . . . . . . . . . . . . . . . 106

7.5 Matching Acute Rheumatic Fever Data . . . . . . . . . . . . . . . . 109

7.6 Reducing Rheumatic Fever with Treatment of Strep Throat . . . . 113

7.7 Conclusions . . . . . . . . . . . . . . . . . . . . . . . . . . . 114

\begin{tabular}{ll}
\hline Appendix A Matlab Code & 118
\end{tabular}

A.1 SIR and SIS Stochastic Plots . . . . . . . . . . . . . . . . . . . . 118

A.2 SIAS Plots . . . . . . . . . . . . . . . . . . . . . . . . 122

A.3 New Zealand Specific Model . . . . . . . . . . . . . . . . . . . . . . 133 
\begin{tabular}{lr}
\hline Bibliography & 137
\end{tabular} 


\section{List of Figures}

$2.1 \quad$ Flow chart for the SIR epidemic model given in equations $|2.1|$ and

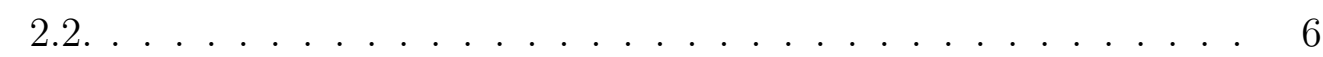

$2.2 \quad$ Flow chart for the SIS epidemic model given in 2.12 . . . . . . . 10

\begin{tabular}{|ll|l|l|l|}
\hline 2.3 & Curves in the $(S, I)$ plane produced by equation & 2.16 & $S_{0}=N$ was \\
\hline
\end{tabular} used to find $c$, with $N=200, \beta=0.01$ and $\gamma=0.5,0.8,1,1.5$. The

\begin{tabular}{|c|}
\hline dashed line shows the maximum possible value of $I$ which occurs as \\
\hline$\frac{\gamma}{\beta} \longrightarrow 0 . \ldots \ldots \ldots \ldots \ldots \ldots \ldots \ldots \ldots \ldots \ldots \ldots \ldots \ldots \ldots$ \\
\hline
\end{tabular}

$2.4 \quad$ Flow chart for the SIR epidemic model with vital dynamics and a constant population size, given by equations $\mid 2.34$ and $\mid 2.35$ [ . . . . . 21

2.5 Three sample paths of the DTMC SIS model in $|2.41|$ The dashed line shows the deterministic model. The different colours show the different sample paths. For all three sample paths the time step $\Delta t=0.01, N=100, \beta=1, \mu=0.1, \gamma=0.25 . \ldots \ldots \ldots 26$

2.6 Three sample paths of the DTMC SIR model in $\mid 2.42$. The dashed line shows the deterministic model. The different colours show the different sample paths. For all three sample paths the time step $\Delta t=0.01, N=100, \beta=1, \mu=0, \gamma=0.25, . \ldots \ldots \ldots .28$

2.7 Three sample paths of the CTMC SIS model in 2.44 . The dashed line shows the deterministic model. The different colours show the different sample paths. For all three sample paths the time step $N=100, \beta=1, \mu=0.1, \gamma=0.25, \ldots \ldots \ldots \ldots \ldots . \ldots . . .30$ 
3.1 Rheumatic fever rates for 2010 by age group and ethnicity. Rates

\begin{tabular}{|l|}
\hline are per 100000 population. This was sourced from the New Zealand \\
\hline public health observatory website, Notifiable disease page. The \\
\hline numbers of acute rheumatic fever amoung the other ethnicities do \\
\hline not show up here because they are too small, they represent only 6 \\
\hline cases in total or 0.000076 per 100000 individuals. . . . . . . . . . . . 38
\end{tabular}

3.2 Rheumatic fever cases by month since 1997. This was sourced from the New Zealand public health observatory website, Notifiable dis-

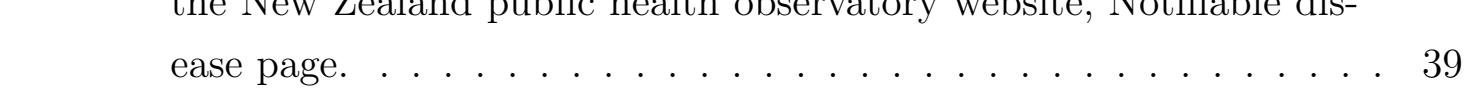

3.3 Rheumatic fever cases by month since 1997 summed together. Data \begin{tabular}{|c|}
\hline were sourced from the New Zealand public health observatory web- \\
\hline
\end{tabular}

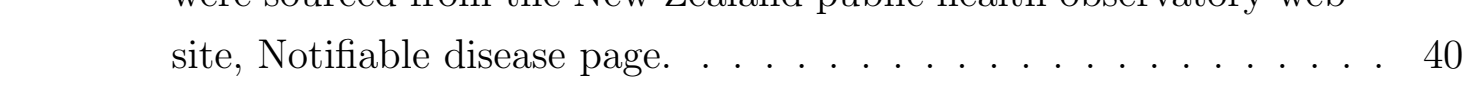

4.1 Flow chart for the SIAS epidemic model, given by 4.1 , showing \begin{tabular}{l}
\hline the rate of movement between compartments as individuals move \\
\hline from Susceptible, though Infectious to developing Acute rheumatic \\
\hline fever, and the different paths they can take back to the Susceptible \\
\hline compartment. . . . . . . . . . . . . . . . . . . . . . . . . . . . . . . 42
\end{tabular}

\begin{tabular}{|lll}
4.2 & A plot of a numerical solution to the SIAS model & 4.1 . with \\
\hline
\end{tabular} \begin{tabular}{|c|}
\hline$N=100, \beta=0.01, \gamma=0.4, \omega=0.2, \kappa=0.8$ and 2 initial in- \\
\hline
\end{tabular} fectious individuals. Under these conditions the numbers of Group

\begin{tabular}{|c|}
\hline A streptococcus (Strep $=I)$ and acute rheumatic fever $(\mathrm{ARF}=A)$ \\
\hline head
\end{tabular}

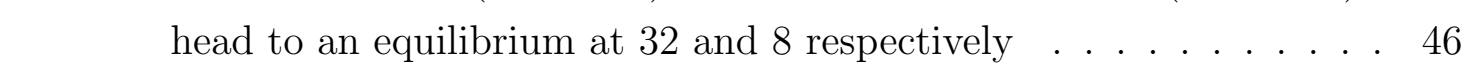

$4.3 \quad$ A plot of $I$ against $S$ for SIAS model. $N=100, \beta=0.1, \gamma=$ \begin{tabular}{|c|c|}
\hline $0.4, \omega=0.2, \kappa=0.8$ with 2 initial infectious individuals. The \\
\hline
\end{tabular} line curves down before we reach $S=0$ as this can only happen if

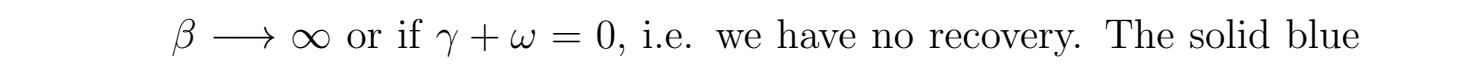
\begin{tabular}{|c|c|c|}
\hline line shows the direct line between $(S, I)=(0, N)$ and $(S, I)=(N, 0)$ & 48
\end{tabular}

4.4 Flow chart for the SIAS DTMC model showing the transition probabilities for moving between each compartment. . . . . . . . . . . 50 
4.5 Two sample paths of the DTMC SIAS model given in 4.9 , The solid lines are the DTMC sample paths. The dashed lines show

\begin{tabular}{|l|}
\hline the deterministic model. The lower set of lines is the number of \\
\hline individuals with acute rheumatic fever $(A)$ and the higher are the \\
\hline number with Group A streptococcus $(I) . N=100, \beta=0.002, \gamma=$ \\
\hline $0.5 . \omega=0.2, \kappa=0.8 \ldots \ldots \ldots \ldots \ldots \ldots \ldots$ \\
\hline
\end{tabular}

\begin{tabular}{|lll}
\hline 4.6 & Two sample paths of the CTMC SIAS model seen in & 4.11 . The \\
\hline
\end{tabular} solid lines are the CTMC sample paths. The dashed lines show

\begin{tabular}{|l|}
\hline the deterministic model. The lower set of lines is the number of \\
\hline individuals with acute rheumatic fever $(A)$ and the higher are the \\
\hline number with Group A streptococcus $(I) . N=100, \beta=0.02, \gamma=$ \\
\hline $0.5 . \omega=0.2, \kappa=0.8 \ldots \ldots \ldots \ldots \ldots$ \\
\hline
\end{tabular}

5.1 Flow chart for the $S I A S$ epidemic model with vital dynamics given by

5.2 Two sample paths of the CTMC SIAS model given by the tran\begin{tabular}{l}
\hline sition probabilities in 5.5 . The solid lines are the CTMC sample \\
\hline paths. The dashed lines show the deterministic model. The lower \\
\hline set of lines is the number of individuals with acute rheumatic fever \\
\hline (A) and the higher are the number with Group A streptococcus (I). \\
\hline$N=100, \beta=0.02, \mu=0.1, \gamma=0.5 . \omega=0.2, \kappa=0.8 \ldots \ldots$ \\
\hline
\end{tabular}

5.3 Flow chart for the $S I A S$ epidemic model with migration and vital dynamics, given in 5.7 . . . . . . . . . . . . . . . . 59

5.4 Flow chart for the SIAS epidemic model with immigration proportional to total population size as given in 5.12 . . . . . . . . . 63

5.5 A flow chart for the $S I R$ model with vaccination. $\kappa$ represents the reduced susceptibility of vaccinated individuals. $\varepsilon$ is the factor by which their infectiousness is reduced. $\lambda$ is the rate of vaccination. . 66 
5.6 A flow chart for the $S I R$ model with treatment as seen in 5.18 .

\begin{tabular}{|c|}
\hline$\delta$ is the factor by which infectiousness is reduced for individuals \\
\hline in treatment. $\alpha$ is the rate of treatment. $\gamma$ and $\eta$ represent the \\
\hline
\end{tabular}

$5.7 \quad$ Flow chart for an acute rheumatic fever model with compartments for individuals with a history of Rheumatic fever. . . . . . . . . . . 69

5.8 Flow chart for an acute rheumatic fever model with compartments

for individuals with a history of acute rheumatic fever and vital

5.9 A plot of numerical solutions for acute rheumatic fever history model given in 5.21 . The dashed, green line represents total Group

\begin{tabular}{|l|}
\hline A streptococcus numbers, both compartment $I$ and $I_{A}$ added to- \\
\hline gether. The solid line plots the acute rheumatic fever $(A)$ numbers. \\
\hline$N=100, \beta=0.02, \mu=0.01, \gamma=0.5 . \omega=0.02, \kappa=0.8, \chi=\ldots \ldots \ldots \ldots$ \\
\hline $0.4, \sigma=0.5 . \ldots \ldots \ldots \ldots$ \\
\hline
\end{tabular}

6.1 Flow of individuals in an acute rheumatic fever treatment model with compartments for individuals with a history of acute rheumatic fever, as modelled by the equations in 6.1$] \ldots$. . . . . . . . . 77

6.2 Flow chart for an acute rheumatic fever treatment model with vital dynamics and compartments for individuals with a history of acute rheumatic fever. . . . . . . . . . . . . . . . 79

6.3 Numerical solution for the model given in 6.2 , Group A streptococcus $\left(I+I_{A}\right)$ is represented by the solid line and acute rheumatic fever $(A)$ numbers are given by the dashed line. $\mathcal{R}_{0}<1$. The initial number of infections was 2. $\mathrm{N}=400, \beta=0.00055, \mu=0.01, \phi=$ $0.1, \omega=0.03, \gamma=0.1, \sigma=0.8 \ldots \ldots \ldots \ldots 1$

6.4 Plot of the reproduction number with $\mathcal{R}_{0}<1$, using numerical solutions to the model given in 6.2 . The same parameters as used in Figure 6.3 apply. . . . . . . . . . . . . . . . . . . . . . . . . . . . . 82 
6.5 Group A streptococcus and acute rheumatic fever numbers, given by numerical solutions to the model seen in $6.2, \quad \mathcal{R}_{0}>1$. The dashed line represents rheumatic fever and the solid line gives Group A streptococcus numbers. The initial number of infectious was 2. $\mathrm{N}=400, \beta=0.0009, \mu=0.01, \phi=0.1, \omega=0.03, \gamma=0.1, \sigma=0.8 \quad 83$

6.6 Plot of the running reproduction number for $\mathcal{R}_{0}>1$, using numerical soulutions to the model given in 6.2 . The same parameters as used in Figure 6.5 apply. . . . . . . . . . . . . . . . . . . . . . . . . 83

6.7 $\quad$ CTMC plot using the transition probabilities given in table 6.1 . The parameter values are the same as those used to produce the plot in figure 6.5. The dashed lines show the deterministic model. ' $I$ ' is the number of individuals infected with Group A streptococcus, ' $A$ ' is the number of indiviuals suffering from acute rheumatic fever. . . 85

6.8 Flow chart for an acute rheumatic fever model including ethnicity, given by the equations in 6.6 . . . . . . . . . . . . . . 87

6.9 Flow chart for an acute rheumatic fever model including ethnicity and rheumatic fever history. . . . . . . . . . . . . . . . . . . . . . . 89

6.10 Plot of a numerical solution to the model seen in 6.9 , Group A streptococcus numbers are represented by the solid line, acute rheumatic fever numbers are given by the dashed line. $\beta=0.00035, \gamma=$ $0.1, \omega=0.006, \lambda=0.3, \mu=0.01, \phi=0.1, \rho=0.1, \kappa=$ $0.025, \eta=0.02, \alpha=0.003, \theta=0.001, N=4000 . \ldots \ldots . . . .92$

6.11 The algorithim from the New Zealand Guideline for Sore Throat Management. . . . . . . . . . . . . . . . . . . . . . 94

6.12 Flow chart for an acute rheumatic fever model including ethnicity and rheumatic fever history. The arrows relating to vital dynamics and ageing have been left out for simplicity in the diagram . . . . . 99 
7.1 Plot of a numerical solution for the model described in $|6.10|$ and

\begin{tabular}{|c|c|}
\hline & $\begin{array}{l}\text { Figure }|6.12| \\
\text { Group A streptococcus numbers are represented by }\end{array}$ \\
\hline & the dashed line and acute rheumatic fever numbers by the solid \\
\hline & line. The parameter values are defined in the above sections. \\
\hline 7.2 & $\begin{array}{l}\text { Numerical solutions for the model in }|6.10| \text { Group A streptococcus } \\
\end{array}$ \\
\hline & numbers a represented by the dashed line and acute rheumatic fever \\
\hline & numbers by the solid line. The parameter values are those defined \\
\hline & in the above sections. $\beta=7 \times 10^{-8}, \kappa=2 \times 10^{-8}$ a \\
\hline
\end{tabular}

7.3 The effective reproduction number, given by the numerical solution to the model in $|6.10|$ using the same parameters as the plot in figure

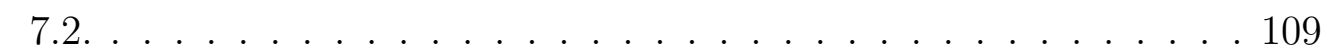

7.4 The effective reproduction number, given by the numerical solution to the model in 6.10 and using $\beta=1.123 \times 10^{-7}, \kappa=2.135 \times$ $10^{-8}$ and $\gamma=1.185 \times 10^{-8}$, with the other parameter values as defined above. . . . . . . . . . . . . . . . . . . . . 110

7.5 Acute rheumatic fever numbers given by the numerical solution to $6.10 \mid$ with $\gamma=1.4 \times 10^{-8} . \ldots \ldots \ldots \ldots 110$

7.6 The solid line shows rheumatic fever numbers, given by the numerical solution to the model in $\mid 6.10$, with $\gamma=1.345 \times 10^{-8}$. The dashed line shows the number of individuals infected with Group A streptococcus. . . . . . . . . . . . . . . . . . . 111

\begin{tabular}{|ll|l|l|}
\hline 7.7 & Rheumatic fever numbers, given by the numerical solution to $6.10 \mid$ \\
\hline
\end{tabular} with $\gamma=1.345 \times 10^{-8}, \omega=0.021, \alpha=0.001$ and $\varphi=0.0001$. . . 112

7.8 Acute rheumatic fever numbers, given by a numerical solution to the model in $\mid 6.10$, with $\omega=0.000022, \alpha=0.000004$ and $\varphi=0.0000004$. 112

7.9 Rheumatic fever numbers given by the numerical solution to 6.10 , with treatment rates $r=0.2$ and $\theta=q=0$. Infection rates and other parameters are the same as in figure 7.8 [ . . . . . . . . . 114 
7.10 Acute rheumatic fever numbers, given by the numerical solution to 6.10 , with treatment rates $r=0.1, \theta=0.6$ and $q=0$. All other parameters are the same as in figure 7.8 . . . . . . . . . . . . . . 115

7.11 The reproduction number given by the numerical solution to $6.10 \mid$ with treatment rates $r=0.1, \theta=0.6$ and $q=0$. All other parameters are the same as in figure 77.8 . . . . . . . . . . . . . . . . . . . 115 


\section{Chapter 1}

\section{Introduction}

Acute rheumatic fever is an autoimmune response to an infection by Group A Streptococcus. This means the immune system creates antibodies in response to the infection, but as well as attacking the infecting bacteria, certain body tissues are also damaged. Tissue such as cardiac tissue, joints, skin, and the central nervous system are affected in particular [11]. Repeat attacks of acute rheumatic fever can lead to chronic rheumatic heart disease and permanent damage to the heart valves [18].

Internationally acute rheumatic fever is considered rare in developed countries [31], but is still acknowledged as highly prevalent in developing countries [11]. Up until the late 1980's its eradication in western developed countries was considered a very real possibility. The decline of acute rheumatic fever, however was halted by the reappearance of isolated out-breaks in the United States [1].

Acute rheumatic fever is a significant problem in New Zealand, particularly among Maori and Pacifica peoples between the ages of 5 and 45. New Zealand maintains some of the highest rates of acute rheumatic fever in a developed country. The respective rates for Maori and Pacific Islanders equate to those of many developing countries.[18].

We can use mathematical models as a way of estimating how different rates of infection, individual interaction and treatment programs might affect the spread of a disease. In using mathematical models we can save time, as we can plot estimates 
for different scenarios much more quickly than the the time disease might actually take to run its course in the population. They also allow us to overcome some of the obvious ethical issues surrounding experimenting with diseases.

In this thesis I provide a background in mathematical modelling of infectious diseases and how these models might apply to acute rheumatic fever. I also develop a model in stages, that can follow the behaviour of acute rheumatic fever in New Zealand and account for the different factors that are specific to this population. I then discuss how this kind of model could be useful in developing realistic treatment programs and reducing the incidence of acute rheumatic fever in New Zealand. 


\section{Chapter 2}

\section{Infectious Disease Modelling}

Mathematical modelling in epidemiology provides an understanding of the underlying mechanisms involved in the spread of a disease that may often be missed in an analysis of experimental data. Models can also help us suggest appropriate control strategies. For endemic diseases the possibility of controlling or even eradicating a disease that has been persistent in the population is worthy of study. [7].

Mathematical modelling of biological systems, including epidemics, is often successfully attacked in steps, starting simply and adding complexity as needed to better model real data [7]. In an epidemiological model we aim to answer several questions about the possible severity of the epidemic. We want to know the total number of individuals who would be affected and how many could need care at any point in time. Other details such as how long the epidemic might last and how much good quarantine would do are also of interest.

There is always a tradeoff between simplicity and detail. Simple models are of value as they are often the building blocks of more complicated models. They can also be useful in highlighting general qualitative behaviour. More detailed models are often designed for specific situations. They are generally difficult to solve analytically however, which limits how useful they can be. Focusing on the asymptotic behaviour of the model, as $t \longrightarrow \infty$, as opposed to finding explicit solutions, can be a good option here too [7]. Though the asymptotic values may 
not always be realistically reached.

A lot of mathematical modelling work has been done in relation to measles epidemics. Because measles has easily recognisable symptoms, and most cases in developed countries are seen by doctors, the data available are often very good [7. Data present a bit more of a problem for modelling acute rheumatic fever. While acute rheumatic fever is a notifiable disease in New Zealand, which provides us with a good record, the infection data available for Group A streptococcus infections are much more limited. This makes it more challenging to validate a model.

\subsection{Compartmental Models}

In most epidemic models, the population is divided into compartments. Individuals are sorted according to their 'state' in relation to the disease [7]. There are three main types of infectious diseases, those caused by viruses, those caused by bacteria and those caused by parasites [13].

Viral infections, such as influenza, measles and chicken pox, usually confer some sort of immunity to reinfection once an individual recovers [7]. These types of infections are best modelled by $S I R$ type models. $S$ stands the number of individuals who are susceptible to infection and could become infected if exposed to the disease. $I$ stands for the number of individuals who are currently infected and may infect susceptible individuals given contact. $R$ stands for Removed; this compartment contains all the individuals who are immune to the disease or otherwise isolated from possible infection. Removed individuals no longer affect the transmission of the disease.

Some diseases, including Group A streptococcus, do not confer any immunity against reinfection [7]. These types of diseases are best modelled with SIS type models. Upon recovery, individuals move back into the Susceptible compartment as they are once again susceptible to infection. 


\subsubsection{Biological Accuracy}

When developing a model, we need to be careful and pay attention to what is going on biologically. Control strategies based on false models can sometimes do more harm than good [7]. One of the most famous epidemics of all time is the Great Plague of London, 1665 - 1666. The simple SIR model can fairly accurately match the exponential rise, turnover and fall of the number of deaths due to the plague per week [7, 16]. There are, however, some features of the data such as jagged oscillations, that remain unexplained by this basic model [7]. If the equations of the $S I R$ model are replaced with stochastic processes, these can be accounted for in the model. Even with the stochastic reformulation however, the longer term re-emergences and extinctions of the plague cannot be explained by the simple $S I R$ model[7]. Biology shows, that the disease primarily infects rodents and is spread by fleas. It is not until we take into account rodents, and the fact that they may carry the disease when it is absent among the human population then cause a re-emergence, that the overall pattern of the plague begins to make sense [20]. This means that placing an infected community under quarantine will not prevent the spread of disease to other communities. To build an accurate model of plague transmission, the populations of fleas and rats, as well as movement in space, needs to be taken into account [7]. Biological accuracy is very important when developing a model for an infectious disease.

\subsubsection{The SIR Model}

In the $S I R$ model the total population size is $N=S+I+R$. Although the numbers of individuals in each compartment must be integers, with sufficiently large population sizes, we can treat $S, I$ and $R$ as continuous variables [7]. This allows us to use differential equations where the rates of transfer between compartments are derivatives of the compartment size, with respect to time [7]. The system of differential equations for the basic SIR model is as follows

$$
\frac{d S}{d t}=-\beta S I
$$




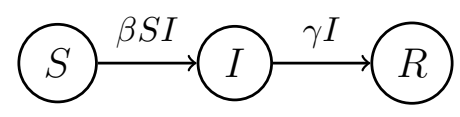

Figure 2.1: Flow chart for the $S I R$ epidemic model given in equations 2.1 and 2.2 .

$$
\frac{d I}{d t}=\beta S I-\gamma I
$$

Where $\gamma$ is the recovery rate. $1 / \gamma$ is the time it takes for each individual to be removed, i.e. it is the average length of time an individual is infectious for. $\beta S I$ describes the rate at which individuals are being infected. It makes sense that the number of individuals being infected per unit of is proportional to both the number of susceptible individuals $(S)$ available to be infected and the number of infectious individuals $(I)$ that are around to infect them. $\beta$ is the proportionality constant, it represents the transmission rate per capita. $\beta$ is dependent on the infectivity of the disease in question as well as characteristics of the population we are dealing with [7]. The fact that $R$ does not appear in either of the equations above reflects the fact that removed individuals do not affect disease transmission [7]. Removed individuals are those who have either recovered from the disease with full immunity, been isolated from the rest of the population somehow, been immunised against infection, or have died as a result of the disease. For the simple $S I R$ model removal is generally seen as recovery from the disease, though isolation due to infection could also be included as part of this [7]. So the equation for $R$ is simply the rate of removal of infectives.

$$
\frac{d R}{d t}=\gamma I
$$

The flow chart in figure 2.1 illustrates how individuals move between the compartments.

The system of equations 2.1, 2.2 and 2.3 forms the Kermack-McKendrick ODE model. This is a special case of the Kermack-McKendrick model, which is formulated in terms of integral equations [13] and used to model the incidence of infection [28]. The Kermack-McKendric model is given in equations 2.4 and 2.5 
below

$$
\begin{aligned}
i(t) & =\alpha(t)+S(t) \int_{0}^{\infty} p(\tau) C(\tau) i(t-\tau) d \tau \\
S(t) & =S(0)-\int_{0}^{t} i(\tau) d \tau
\end{aligned}
$$

$t$ is the amount of time since the outbreak began, ie. $t=0$ coincides with the first case of the disease in question in the population.

$i(t)$ is incidence of infection, or the number of new infections per unit of time, at time t.

$\alpha(t)$ represents the incidence rate of the index case. This is the number of cases being imported into the population at time $t[28$. The index case is the first case of the infection in a particular epidemic. We can have multiple index cases however if those cases come from outside of the population in which the epidemic is taking place. If $\alpha(t) \neq 0$, the population is not closed. The incidence of the index case is 0 in a closed population.

$S(t)$ is the number of susceptible people in the population at time $t$.

$\tau$ is the time since exposure to the infection. So $\int_{0}^{t} i(\tau) d \tau$ models the total number of cases, that have occurred, since the beginning of the outbreak. $i(t-\tau)$ gives the number of new cases, per unit of time, that were occurring $\tau$ units of time ago.

$p(\tau)$ is the probability that an individual infected $\tau$ units of time ago, will infect another individual, given contact [28]. This is also known as the infectivity of the disease [13].

$C(\tau)$ is the contact rate per host [28].

$S(0)$ is the number of susceptible individuals in the population before the outbreak began. So essentially, $S(0) \equiv N$, the population size.

We can represent the total number of infected individuals at time $t$ by

$$
I(t)=\int_{0}^{\infty} g(\tau) i(t-\tau) d \tau
$$

Where $g(\tau)$ represents the probability an individual is still infectious $\tau$ units of 
time after being infected. The total number of removed individuals at time $\mathrm{t}$ is

$$
R(t)=\int_{0}^{\infty}(1-g(\tau)) i(t-\tau) d \tau
$$

It is dependent on individuals no longer being infectious after $\tau$ units of time.

By assuming a constant contact rate between individuals and a constant 'removal' rate for infectious individuals, we can use 2.4 to derive the Kermack-McKenderick ODE model from the three equations 2.5, 2.6 and 2.7. We are also assuming a closed population with no new individuals entering, and no one leaving the population. This means $\alpha(t)=0$, which is acceptable as long the timescale of the epidemic is significantly shorter than the timescale for births, deaths and migration. Though we still require a source of infection at $t=0$.

$$
\begin{aligned}
\frac{d S}{d t} & =(S(0))^{\prime}-i(t) \\
& =0-\alpha(t)-S(t) \int_{0}^{\infty} p(\tau) C(\tau) i(t-\tau) d \tau
\end{aligned}
$$

$p(\tau) C(\tau)$ represents the rate at which contacts resulting in infection are made by an individual who was infected $\tau$ units of time ago. $\beta$ represents the constant rate a which contacts that would result in infection occur per individual. So $\beta g(\tau)$ represents the rate at which an individual, who was infected $\tau$ units of time ago, makes contacts that result in infection. hence if we assume a constant rate of contact in the population we can use $p(\tau) C(\tau)=\beta g(\tau)$.

$$
\begin{aligned}
\Longrightarrow \frac{d S}{d t} & =-S(t) \int_{0}^{\infty} \beta g(\tau) i(t-\tau) d \tau \\
& =-\beta S(t) I(t) \\
\Longrightarrow i(t) & =\beta S(t) I(t)
\end{aligned}
$$

The results in 2.8 is the same as equation 2.1 28].

If we let $r(\tau)$ be the number of infected individuals who are still infectious $\tau$ units 
of time after infection, assuming a constant rate of removal $\gamma$

$$
\begin{aligned}
\frac{d r}{d \tau} & =-\gamma r \\
\frac{d r}{r} & =-\gamma d \tau \\
\ln (r) & =c-\gamma \tau \\
r(\tau) & =r(0) e^{-\gamma \tau}
\end{aligned}
$$

So if we define the rate of removal to be $\gamma$, we see that the length of the infectious period is exponentially distributed with a mean length of $1 / \gamma$ [7]. So we can substitute $g(\tau)=e^{-\gamma \tau}$ for the probability that an individual infected $\tau$ units of time ago is still infectious, so that

$$
\begin{aligned}
I(t) & =\int_{-\infty}^{t} e^{-\gamma(t-\tau)} i(\tau) d \tau \\
& =e^{-\gamma t} \int_{-\infty}^{t} e^{\gamma \tau} i(\tau) d \tau \\
\Rightarrow \frac{d I}{d t} & =-\gamma e^{-\gamma t} \int_{-\infty}^{t} e^{\gamma \tau} i(\tau) d \tau+e^{-\gamma t} e^{\gamma t} i(t) \\
& =-\gamma I(t)+i(t) \\
& =-\gamma I(t)+\beta S(I)
\end{aligned}
$$

Which is the same as equation 2.2 [28].

This model only makes sense if both $S(t)$ and $I(t)$ are always non-negative. If either of them reaches zero the system, and therefore the epidemic, is considered terminated. We can clearly see from the flow chart in figure 2.1 that the population will continue to flow from $S$, through $I$, to $R$ until either the disease dies out, at $I=0$, or everyone is immune, $R=N$. S is always decreasing and $I$ increases to a maximum before decreasing. When this maximum happens and how big it is are of interest[7].

In using this model we assume that each individual makes contact, capable of resulting in infection, with $\beta N$ other individuals per unit time and that infective individuals are removed at a rate $\gamma I$ per unit of time. While the assumption of a 


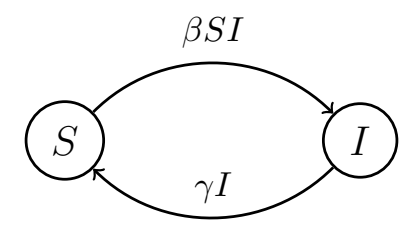

Figure 2.2: Flow chart for the SIS epidemic model given in 2.12.

contact rate proportional to population size is unrealistic, except near the start of an epidemic, many more realistic models will exhibit similar qualitative behavior [7].

\subsubsection{The SIS Model}

Some diseases, particularly those caused by bacterial infections, confer no immunity on individuals who have recovered [7]. Upon recovery individuals move from being infected back to being susceptible. Group A streptococcus is one such infection. To account for this in a model, we can modify the SIR model by removing the equation for $R$ and adding the term describing rate of recovery to the equation for $S$. This gives us the SIS model below

$$
\begin{aligned}
& \frac{d S}{d t}=-\beta S I+\gamma I \\
& \frac{d I}{d t}=\beta S I-\gamma I
\end{aligned}
$$

Figure 2.2 shows the flow of infectious individuals between the Susceptible and infectious compartments [7]. In this type of model we have $N=S+I$.

\subsection{The basic reproduction number $\mathcal{R}_{0}$}

$\mathcal{R}_{0}$ is the basic rate of disease spread at time zero with no intervention. It is defined as the number of individuals who would be directly infected by an infectious individual released into a fully susceptible population [13]. The value of $\mathcal{R}_{0}$ 
is closely correlated with several important characteristics of an epidemic. The size of peaks in the number of cases and when these peaks occur are two such characteristics.

$\mathcal{R}_{0}$ exhibits a threshold behavior in relation to how likely it is an epidemic will occur. If $\mathcal{R}_{0}>1$ a major epidemic is likely. If $\mathcal{R}_{0}<1$ the disease will likely die out quickly and only a minor epidemic will occur [7].

In estimating $\mathcal{R}_{0}$ we are concerned only with the rate at which individuals enter and exit the infectious compartment. Where they have come from and where they recover to do not affect the reproduction number.

In the $S I S$ and $S I R$ models, for an infectious individual introduced into a totally susceptible population $S(0) \simeq N$. In this case we would expect the initial rate of infection per infected individual, at time $t=0$, to be $\beta N$. That is the infectious individual makes $\beta N$ contacts, per unit of time, where the infection is passed on. The reproduction number of a disease at any point in time is the rate at which an infectious person is infecting others multiplied by how long they are infectious for [7]. So if the mean infectious period is $1 / \gamma$, we would expect

$$
\mathcal{R}_{0}=\frac{\beta N}{\gamma}
$$

$\mathcal{R}_{0}$ can also be found as the largest eigenvalue of the next generation matrix [14].

\subsubsection{The Effective Reproduction Number $\mathcal{R}^{*}(t)$}

We can now also define $\mathcal{R}^{*}$, the time-dependent running reproduction number. It is the effective rate of disease reproduction at time $t$, taking in to account the effects of any intervention as well. $\mathcal{R}^{*}$ represents the number of secondary infections caused by an individual in the population who becomes infectious at time $t$. An infectious individual makes $C$ contacts per unit time, where the infection could be passed on if that contact is made with a susceptible individual. $S / N$ represents the proportion of those contacts that are with susceptible individuals. Using this 
information along with equation 2.13 , for the $S I R$ and $S I S$ models we get

$$
\begin{aligned}
\mathcal{R}^{*}(t) & =\frac{C}{\gamma} \times \frac{S}{N} \\
& =\frac{\beta N}{\gamma} \times \frac{S}{N} \\
& =\frac{\beta S}{\gamma}
\end{aligned}
$$

If $\mathcal{R}^{*}<1$ for all large $t$, the epidemic will die out [7]. If $\mathcal{R}^{*}>1$ then the number of infectious individuals is increasing. Because infectious individuals come from susceptible individuals being infected, and there is no source of new susceptible individuals in this model, an increase in $I$ implies a decrease in $S$. $\beta$ and $\gamma$ are both non-negative constants, so $\mathcal{R}^{*}$ decreases as $S$ decreases. $S$ will continue to decrease as long as individuals continue to become infected. This means that $\mathcal{R}^{*}$ will continue to decrease until the disease dies out. So a disease modelled by the SIR model will always die out [7].

For the SIS model however, individuals recover into the susceptible compartment. In this case, when $\mathcal{R}^{*}>1$ the system will tend towards an equilibrium where the number of susceptible individuals being infected is equal to the number of infectious individuals recovering. This is called the endemic equilibrium, as the infection becomes endemic in the population. For the basic SIS model this equilibrium occurs at $\beta S=\gamma$. So in an $S I S$ system for, $\mathcal{R}^{*}>1, \mathcal{R}^{*}$ decreases as $\mathcal{R}^{*}$ approaches 1 as $t \longrightarrow \infty$.

\subsubsection{Final size}

The final size of an epidemic is the total number of infections caused by a disease before it dies out. This is another characteristic of epidemics that is predictable given $\mathcal{R}_{0}$, or conversely can be used to estimate $\mathcal{R}_{0}$ in retrospect. Though it is only applicable to $S I R$ type models

Even with the basic $S I R$ model, it is not possible to obtain an exact solution for $I(t)$. We can however, solve for $I$ as a function of $S$ by taking the ratio of equations 


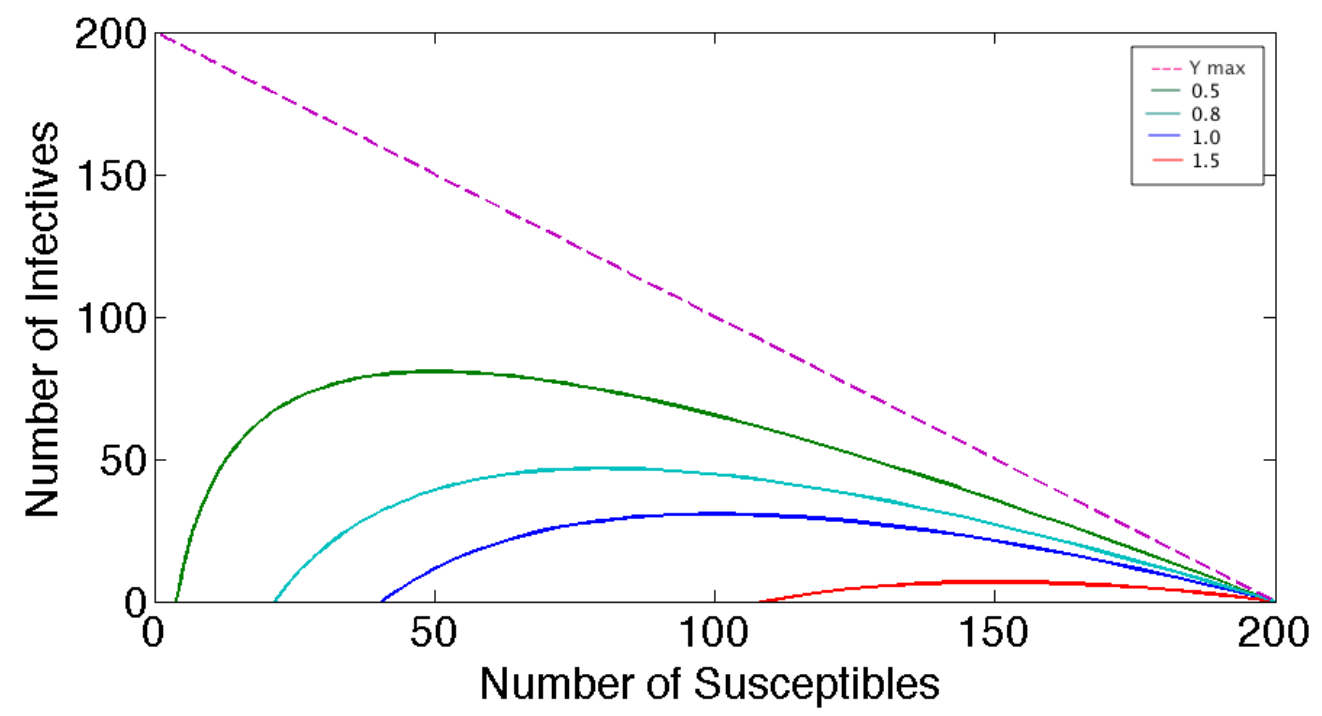

Figure 2.3: Curves in the $(S, I)$ plane produced by equation 2.16. $S_{0}=N$ was used to find $c$, with $N=200, \beta=0.01$ and $\gamma=0.5,0.8,1,1.5$. The dashed line shows the maximum possible value of $I$ which occurs as $\frac{\gamma}{\beta} \longrightarrow 0$.

2.1 and 2.2 [7]

$$
\begin{aligned}
\frac{d I}{d S} & =\frac{\beta S I-\gamma I}{-\beta S I} \\
& =-1+\frac{\gamma}{\beta S}
\end{aligned}
$$

The maximum number of individuals infectious at the same time occurs when the derivative of $I$ is zero, which is when $\beta S=\gamma$ [7].

We can then integrate 2.15 to find curves in the $(S, I)$ plane.

$$
I=-S+\frac{\gamma}{\beta} \ln (S)+c
$$

Where $c$, the arbitrary constant of integration is determined by the initial values $S(0)$ and $I(0)$. Figure 2.3 shows some of these curves with varying values of $\gamma$.

We define the function

$$
V(S, I)=S+I-\frac{\gamma}{\beta} \ln (S),
$$


so that solutions are given by $V(S, I)=c$ for all $t$ [7].

At the start of an epidemic we have a population of size $N$ and the number of susceptible individuals $S_{0} \approx N$. If a small number of infectious individuals are introduced into a large population, we have large $N$, so $I_{0} \approx 0$. By using these two approximations and that $\lim _{t \rightarrow \infty} I(t)=0$, for the $S I R$ model, we can use $V\left(S_{0}, I_{0}\right)=V\left(S_{\infty}, 0\right)=c$ where $S_{\infty}=\lim _{t \longrightarrow \infty} S(t)$ to get

$$
N-\frac{\gamma}{\beta} \ln \left(S_{0}\right)=S_{\infty}-\frac{\gamma}{\beta} \ln \left(S_{\infty}\right)
$$

The contact rate $\beta$ can be difficult to estimate as it depends on the particular disease in question and also social and behavioural factors. We can rewrite 2.17 in terms of $\mathcal{R}_{0}$, using 2.13 , to get

$$
\begin{aligned}
\frac{\gamma}{\beta}\left(\ln S_{0}-\ln S_{\infty}\right) & =N-S_{\infty}, \\
\Rightarrow \quad \ln S_{0}-\ln S_{\infty} & =\frac{\beta N-\beta S_{\infty}}{\gamma} \\
& =\mathcal{R}_{0}-\frac{\beta S_{\infty}}{\gamma}, \\
\Rightarrow \quad \ln S_{0}-\ln S_{\infty} & =\mathcal{R}_{0}\left[1-\frac{S_{\infty}}{N}\right] .
\end{aligned}
$$

We may be able to estimate $S_{0}$ and $S_{\infty}$ using blood samples, of a random sample of the population taken before and after an epidemic. Using equation 2.18, we can estimate what $\mathcal{R}_{0}$ was, for a particular epidemic [7]. If we know the value of $\mathcal{R}_{0}$ and $S_{0}$ we can estimate what the final size of the epidemic might be.

This final size calculation is not practical for SIS type models however. If we take the ratio of the equations in 2.12 we get

$$
\frac{d I}{d S}=\frac{-\beta S I+\gamma I}{\beta S I-\gamma I}=-1
$$

This just implies that $I$ increases at the same rate $S$ decreases and vice versa. 


\subsubsection{Equilibrium Points}

Once a disease dies out, at $I=0$, no new infectious individuals can develop for that particular outbreak. There are no infectious individuals left to infect anyone who is susceptible. This is known as the disease free equilibrium [7].

An endemic equilibrium is when the number of infectious individuals in the population reaches a certain value and remains there. This usually occurs when the rate of infection equals the rate of recovery.

We can find the equilibrium points for a particular system of equations by setting each differential equation in the system equal to zero and solving to find the number of individuals in each compartment[7].

\subsubsection{SIS Equilibrium Points}

For the $S I S$ epidemic model, because $N=S+I$ we can rewrite the system of equations in 2.12 as

$$
\begin{aligned}
\frac{d I}{d t} & =-\beta(N-I) I+\gamma I=0 \\
& \Rightarrow I=0 \text { or } \beta(N-I)=\gamma
\end{aligned}
$$

This gives us the two equilibrium points $(S, I)=(N, 0)$ and $\left(\frac{\gamma}{\beta}, N-\frac{\gamma}{\beta}\right)$.

The stability of an equilibrium point tells us what happens to points close to it. If an equilibrium point is unstable, all nearby points move away from it over time. All points within a certain distance of a stable equilibrium point will move towards it and eventually end up there. In the context of a disease model, the stability of the equilibrium points tells what will happen if introduce the disease to the population. If the disease free equilibrium is stable, the introduced disease will die out, returning the model to the disease free state. If the disease free is unstable however, as soon as we introduce the disease in to the population, we begin to move away from the disease free state and, if it is stable, towards the endemic equilibrium.

Either of these equilibrium points will be stable if the value of $\frac{d^{2} I}{d t^{2}}$ is negative at 
that point.

$$
\frac{d^{2} I}{d t^{2}}=\beta N-2 \beta I-\gamma
$$

The first point, $(S, I)=(N, 0)$ is the disease free equilibrium. For the $S I S$ model there can only be one disease free equilibrium, because once the disease dies out everyone recovers to the susceptible compartment. At this equilibrium point

$$
\begin{aligned}
\frac{d^{2} I}{d t^{2}} & =\beta N-2 \beta(0)-\gamma \\
& =\beta N-\gamma
\end{aligned}
$$

This means the disease free equilibrium is stable as long as $\beta N<\gamma$. When $\beta N<\gamma, \mathcal{R}_{0}=\beta N / \gamma<1$. So when the disease free equilibrium is stable, the value of $\mathcal{R}_{0}$ is also telling us that an epidemic is unlikely and the disease will die out.

The point $(S, I)=\left(\frac{\gamma}{\beta}, N-\frac{\gamma}{\beta}\right)$ is the endemic equilibrium. Because it is at the point where $\beta S=\gamma$ and $S \leq N$, for this equilibrium point to exist and be valid we require $\beta N \geq \gamma$. This means the endemic equilibrium is only valid when the disease free equilibrium is unstable. At the endemic equilibrium we get;

$$
\begin{aligned}
\frac{d^{2} I}{d t^{2}} & =\beta N-2 \beta\left(N-\frac{\gamma}{\beta}\right)-\gamma \\
& =\beta N-2 \beta N+2 \gamma-\gamma \\
& =\gamma-\beta N
\end{aligned}
$$

So the endemic equilibrium is stable as long as $\beta N>\gamma$, i.e when it is valid, $\mathcal{R}_{0}>1$ and the disease free equilibrium becomes unstable. This swapping of stability through the point $\beta N=\gamma$ is known as transcritical bifurcation [5].

\subsubsection{2 $S I R$ Equilibrium Points}

For the $S I R$ model we can use equations 2.1 and 2.2 from section 2.1 .2 to find the equilibrium points. We do not need to use the equation for $R(2.3)$, as $R$ does not appear in any of the equations and $N=S+I+R$ is constant. This means if $I$ 
and $S$ are not changing, $R$ cannot be changing either. Given $N, S$ and $I, R$ is easily determined [7].

$$
\begin{aligned}
& \frac{d I}{d t}=\beta S I-\gamma I=0 \\
& \quad \Rightarrow I=0 \text { or } \beta S=\gamma
\end{aligned}
$$

Setting $I=0$ results in all three variables being stationary, $\frac{d S}{d t}=\frac{d I}{d t}=\frac{d R}{d t}=0$, thus satisfying the conditions for equilibrium. Setting $\beta S=\gamma$ results in $\frac{d S}{d t}=$ $-\gamma I=-\frac{d R}{d t}$, which requires $I=0$ for the system to be in equilibrium. $I=0$ is the disease free equilibrium, regardless of the value of $S$. There are no other equilibrium points in this system.

\subsubsection{The Jacobian Matrix and Stability}

Given a system of equations $f\left(x_{1}, x_{2} \ldots x_{n}\right)$ the Jacobian matrix is the $n \times n$ matrix of partial derivatives [26].

$$
J\left(x_{1}, x_{2} \ldots x_{n}\right)=\left[\frac{\partial f_{i}}{\partial x_{j}}\right]
$$

The Jacobian matrix for this system in 2.23 is given by

$$
J(S, I)=\left[\begin{array}{ll}
\frac{\partial\left(\frac{d S}{d t}\right)}{\partial S} & \frac{\partial\left(\frac{d S}{d t}\right)}{\partial I} \\
\frac{\partial\left(\frac{d I}{d t}\right)}{\partial S} & \frac{\partial\left(\frac{d I}{d t}\right)}{\partial I}
\end{array}\right]=\left[\begin{array}{cc}
-\beta I & -\beta S \\
\beta I & \beta S-\gamma
\end{array}\right]
$$

If all the eigenvalues of the Jacobian matrix at the equilibrium point $\overline{\mathbf{x}}$ have negative real parts, then the equilibrium point $\overline{\mathbf{x}}$ is asymptotically stable [17].

The disease free equilibrium point, $(S, I)=(S, 0)$, represents the situation where the disease has died out after infecting a number of people who have now recovered and moved into compartment $R$. $S$ is the number of Susceptible individuals left 
who were never infected. The Jacobian matrix at this point becomes

$$
\left[\begin{array}{cc}
0 & -\beta S \\
0 & \beta S-\gamma
\end{array}\right]
$$

The eigenvalues of this matrix are 0 and $\beta S-\gamma$. This means the disease free equilibrium is stable when $\beta S<\gamma$, which implies the rate of infection is less than the rate of recovery. Because $S$ is reduced in size as individuals become infected, in the situation a disease is introduced where $\beta S \geq \gamma$, the disease free equilibrium is initially unstable and we move away from it, until $S$ is reduced to the point where $\beta S<\gamma$. At this point the disease free equilibrium becomes stable and the disease starts to die out, returning the system to the disease free state.

This makes sense as $\beta S>\gamma$ implies $\frac{\beta S}{\gamma}>1$, or at time $t=0, \mathcal{R}_{0}>1$, which would suggest an epidemic will occur.

\subsubsection{Herd Immunity}

Herd immunity occurs when some fraction of the population being immune protects the whole population against an outbreak becoming endemic. If a fraction of the population, $p$, is successfully immunised, we essentially replace $N$ with $N(1-p)$ and therefore $\mathcal{R}_{0}$ with $\mathcal{R}_{0}(1-p)$. Then for a disease to die out we then require $\mathcal{R}_{0}(1-p)<1$, or

$$
\begin{aligned}
1-p & <\frac{1}{\mathcal{R}_{0}} \\
p & >1-\frac{1}{\mathcal{R}_{0}}
\end{aligned}
$$

Smallpox is the only disease for which herd immunity has been achieved worldwide. $\mathcal{R}_{0}$ for smallpox is approximately 5 so an $80 \%$ minimum rate of immunisation was required to achieve this [7]. 


\subsection{Numerical Approximations}

Initially the number of infective individuals grows exponentially and with a large enough population size, we can approximate the equation for $I$, in either the $S I S$ or $S I R$ model, by

$$
\begin{aligned}
\frac{d I}{d t} & =\beta N I-\gamma I \\
& =(\beta N-\gamma) I
\end{aligned}
$$

So the initial rate of growth $(g)$, of infected individuals is

$$
\begin{aligned}
g & \equiv \frac{1}{I} \frac{d I}{d t} \\
g & =\beta N-\gamma \\
& =\frac{\gamma(\beta N-\gamma)}{\gamma} \\
g & =\gamma\left(\mathcal{R}_{0}-1\right)
\end{aligned}
$$

This growth rate $g$ can be estimated experimentally near the beginning of an epidemic, based on sampling of initial cases seen by health professionals. We could then calculate $\beta$ as

$$
\beta=\frac{g+\gamma}{N}
$$

This estimate should be used cautiously however. It is likely to be very inaccurate, due to incomplete data, underreporting and misdiagnosis of cases, especially when dealing with the outbreak of a new disease [6].

Even with the simple system of equations in the basic SIR model, we cannot solve exactly for $I(t)$ as an expression of $t$. We can however find a numerical approximation by using small time intervals of $\Delta t$ and a computer with Euler's Method [15]. Though it is not the only way, Euler's method is a simple way to solve for the number of susceptible individuals $\Delta t$ time units into the future [7]. Using $\Delta S=S(t+\Delta t)-S(t)$ and the approximation $d S / d t \simeq \Delta S / \Delta t$

$$
\frac{\Delta S(t)}{\Delta t} \simeq-\beta S(t) I(t)
$$




$$
\begin{aligned}
\frac{S(t+\Delta t)-S(t)}{\Delta t} & =-\beta S(t) I(t) \\
S(t+\Delta t)-S(t) & =-\beta S(t) I(t) \Delta t \\
S(t+\Delta t) & =S(t)-\beta S(t) I(t) \Delta t
\end{aligned}
$$

In the same way the number of infectives at time $t+\Delta t$ is

$$
I(t+\Delta t)=I(t)+\beta S(t) I(t) \Delta t-\gamma I(t) \Delta t
$$

Initial conditions for $I$ and $S$ are important and can make a huge difference to the resulting model. For a disease that is persistent in the population we cannot assume $S(0)=N$ and $I(0)=1$ as we have no idea when or even if this was ever the case. We must instead use data available to us to choose a suitable starting point and use those values for initial conditions [7]. Another method, the Runge-Kutta, method us used in solving many of the later models in this thesis.

\section{$2.4 \quad$ Vital dynamics}

One of the problems with the previous SIR model 2.1 and 2.2 in section 2.1.2, is it does not allow for repeat epidemics. The disease starts to die out after we reach the point $\beta S=\gamma$, as the number of susceptible individuals becomes to small for the the disease to spread as easily. It will cease to exist in the population once all the infected persons recover.

In the case of an put break where demographic and epidemic changes take place over similar timescales we need to include vital dynamics, such as births and deaths in our model.this has the side effect of introducing a source of new susceptible individuals such as newborn babies. We can include the effects of births and deaths in the model with modified version of the basic SIR model. These vital dynamics are independent of the disease; they are the rates of birth and death that would exist in the population if the disease in question were absent. Assuming that all newborns enter the population as susceptible individuals, if we let $B$ be 


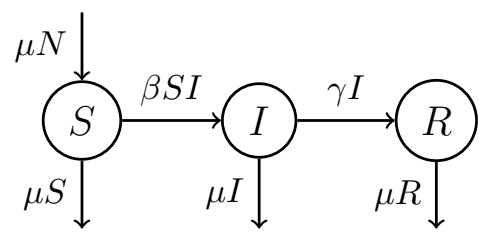

Figure 2.4: Flow chart for the SIR epidemic model with vital dynamics and a constant population size, given by equations 2.34 and 2.35 .

the birth rate and $\mu$ be the death rate we get

$$
\begin{aligned}
& \frac{d S}{d t}=B-\beta S I-\mu S \\
& \frac{d I}{d t}=\beta S I-\gamma I-\mu I
\end{aligned}
$$

We may also assume a constant population size for the duration of the epidemic, as changes in birth and death rates usually take place over a much larger time frame than an epidemic. So if we maintain a constant population size by setting births equal to deaths we have $B=\mu N$. The flow chart in figure 2.4 shows how individuals move in and out of each compartment when we include vital dynamics in the SIR model and keep the population constant.

By including these vital dynamics we can produce oscillations in our model that correspond to recurrent epidemic data seen in real populations. However, unlike the oscillations seen in the data, our oscillations that occur solely with the introduction of vital dynamics damp out over time to eventually reach an endemic equilibrium. In fact it can be proven that this equilibrium is globally asymptotically stable. So no matter what the starting conditions or the values of the other parameters are, the solutions will always converge on the equilibrium. Because of this, the basic SIR model with vital dynamics alone is not enough to explain the oscillations seen in the data for most recurring epidemics that show a oscillating pattern [7. We may need to introduce more complex elements to the model to more accurately represent the behaviour of an infection in the population. 


\subsection{1 $S I R$ Equilibrium points with vital dynamics}

To find the equilibrium points for the $S I R$ model with vital dynamics, we set equations 2.34 and 2.35 equal to zero. Using $B=\mu N$ for a constant population size we solve for $S$ and $I$.

$$
\begin{aligned}
& \frac{d I}{d t}=\beta S I-\gamma I-\mu I=0 \\
& \quad \Rightarrow \beta S=\gamma+\mu \text { or } I=0
\end{aligned}
$$

If we substitute each of these values into equation 2.34 we get the following If $I=0$

$$
\begin{aligned}
\mu N-\mu S & =0 \\
\mu S & =\mu N \\
S & =N
\end{aligned}
$$

If $\beta S=\gamma+\mu$

$$
\begin{aligned}
\mu N-(\gamma+\mu) I-\frac{\mu(\gamma+\mu)}{\beta} & =0 \\
\mu N-\frac{\mu(\gamma+\mu)}{\beta} & =(\gamma+\mu) I \\
I & =\frac{\mu N}{\gamma+\mu}-\frac{\mu}{\beta}
\end{aligned}
$$

So the two equilibrium points are $(N, 0)$ and $\left(\frac{\gamma+\mu}{\beta}, \frac{\mu N}{\gamma+\mu}-\frac{\mu}{\beta}\right)$.

The Jacobian matrix for this system 2.34 and 2.35 is given by

$$
J(S, I)=\left[\begin{array}{ll}
\frac{\partial\left(\frac{d S}{d t}\right)}{\partial S} & \frac{\partial\left(\frac{d S}{d t}\right)}{\partial I} \\
\frac{\partial\left(\frac{d I}{d t}\right)}{\partial S} & \frac{\partial\left(\frac{d I}{d t}\right)}{\partial I}
\end{array}\right]=\left[\begin{array}{cc}
-\beta I-\mu & -\beta S \\
\beta I & \beta S-\gamma-\mu
\end{array}\right]
$$

The disease free equilibrium, at $(N, 0)$ represents a population where the disease in 
question has died out and all those who recovered from the disease have also died, at the usual death rate. As soon as the disease stops spreading we are headed for this equilibrium and the equation for Susceptibles $S$ heads towards an equilibrium at $I=0$. However the number of individuals in the Removed compartment $R$ continues to shrink at the rate $\mu R$, therefore the system does not technically reach equilibrium until $R=0$ as well. The Jacobian matrix at this equilibrium becomes

$$
\left[\begin{array}{cc}
-\mu & -\beta N \\
0 & \beta N-\gamma-\mu
\end{array}\right]
$$

This matrix has eigenvalues $-\mu$ and $\beta N-\gamma-\mu$, so the equation is stable when $\beta N<\gamma+\mu$.

Because $\beta N \geq \beta S=\gamma+\mu$ at the endemic equilibrium, $\left(\frac{\gamma+\mu}{\beta}, \frac{\mu N}{\gamma+\mu}-\frac{\mu}{\beta}\right)$, it is only valid as long as $\beta N \geq \gamma+\mu$. This also ensures that the number of individuals in the infectious compartment remains non-negative. The Jacobian matrix at this equilibrium point becomes

$$
\left[\begin{array}{cc}
-\frac{\beta \mu N}{\gamma+\mu} & -\gamma-\mu \\
\frac{\beta \mu N}{\gamma+\mu}-\mu & 0
\end{array}\right]
$$

The eigenvalues for this matrix are not very simple, however there is another way to determine the stability of an equilibrium point.

Let us define a matrix $M$

$$
M=\left[\begin{array}{ll}
A & B \\
C & D
\end{array}\right]
$$

$M$ has determinant $|M|=A D-B C$ and trace $\operatorname{tr}(M)=A+D$. To find the eigenvalues we find the roots of the characteristic equation given by $|M-\lambda I|=0$, where $I$ is the identity in this case and $\lambda$ is an eigenvalue.

$$
\begin{aligned}
|M-\lambda I| & =0 \\
\Rightarrow(A-\lambda)(D-\lambda)-B C & =0 \\
\Rightarrow \lambda^{2}-\lambda(A+D)+A D-B C & =0 \\
\Rightarrow \lambda^{2}-\operatorname{tr}(M) \lambda+|M| & =0
\end{aligned}
$$


So if $\operatorname{tr}(M)<0$ and $|M|>0$ then the roots of the characteristic equation 2.40, and hence the eigenvalues of $M$ both have negative real parts [13].

The Jacobian matrix for the endemic equilibrium in this system 2.38 has determinant $\beta \mu N-\mu(\gamma+\mu)=\mu(\beta N-\gamma-\mu)$ which is positive if $\beta N>\gamma+\mu$.

The trace is $-\frac{\beta \mu N}{\gamma+\mu}$, which is always negative as all of the parameters and compartment sizes are non-negative.

So the endemic equilibrium is stable as long as $\beta N>\gamma+\mu$. This is similar to analysis seen in Brauer [7].

\subsection{Stochastic Epidemic Models}

In the previous sections, by using differential equations we are treating $I$ and $S$ as continuous variables. As discussed previously this is quite reasonable for a large population size $N$. However this assumption still has some observable effect on the predictions of the model. By adding a small amount of noise to the model, or replacing it with a stochastic version, we can account for the fact that $I$ and $S$ are actually discrete variables, or that some things don't happen deterministically. Doing this also prevents the oscillations in the previous model 2.34 and 2.35 from damping out [7].

\subsubsection{Discrete Time Markov Chain Models}

Using Markov chains, we can derive realistic stochastic models that are based on our deterministic models. Let $p_{j i}=$ the probability of transition from state $I(t)=i$ to state $I(t+\Delta t)=j$ during the time interval $\Delta t$. This is the probability that the number of infectious individuals changes from $i$ to $j$ over the time step $\Delta t$. For a simple discrete time model we choose $\Delta t$ to be small enough that at most one transition can take place during the time step. This means $j=i-1, j=i+1$ or $j=i[7]$. 


\subsubsection{The SIS DTMC model}

For the SIS epidemic model, using a Discrete Time Markov Chain or DTMC model, the transition probabilities look like this

$$
p_{j i}(\Delta t)= \begin{cases}\beta i(N-i) \Delta t & j=i+1 \\ (\mu+\gamma) i \Delta t & j=i-1 \\ 1-[\beta i(N-i)+(\mu+\gamma) i] \Delta t & j=i \\ 0 & \text { otherwise }\end{cases}
$$

This shows the probabilities for each possible change in the number of infectious individuals over one time step $\Delta t$. The probabilities are based on the differential equations for the SIS model with vital dynamics, $\mu=$ birth-rate $=$ death-rate. The probability that the number of infectious individuals decreases by one is the sum of the probability that an infectious individual dies and the probability that an infectious individual recovers. These probabilities are determined by the deathrate $\mu$ and the recovery rate $\gamma$ in the differential equations for the SIS model with vital dynamics.

Figure 2.5 shows a plot of some sample paths produced by the SIS DTMC transition probabilities, along with the curve produced by the deterministic SIS model. The sample paths were produced in Matlab, a random number between 0 and 1 , was chosen from a uniform distribution and used with the transition probabilities in 2.41, to decide the change in the number of infectious individuals at each time step. The code for how this was done is given in Appendix A.1.

Two of the sample paths presented in Figure 2.5 follow the deterministic plot, fairly well, towards the endemic equilibrium. The green path however, shows the number of infected individuals dying out near the start of the plot, leaving us with the disease free state. This is an example of one characteristic of stochastic epidemic models, there is always the possibility that the disease will start die out, from any point in the epidemic, reaching in the disease free equilibrium regardless of the parameter values and what is predicted by the deterministic model. 


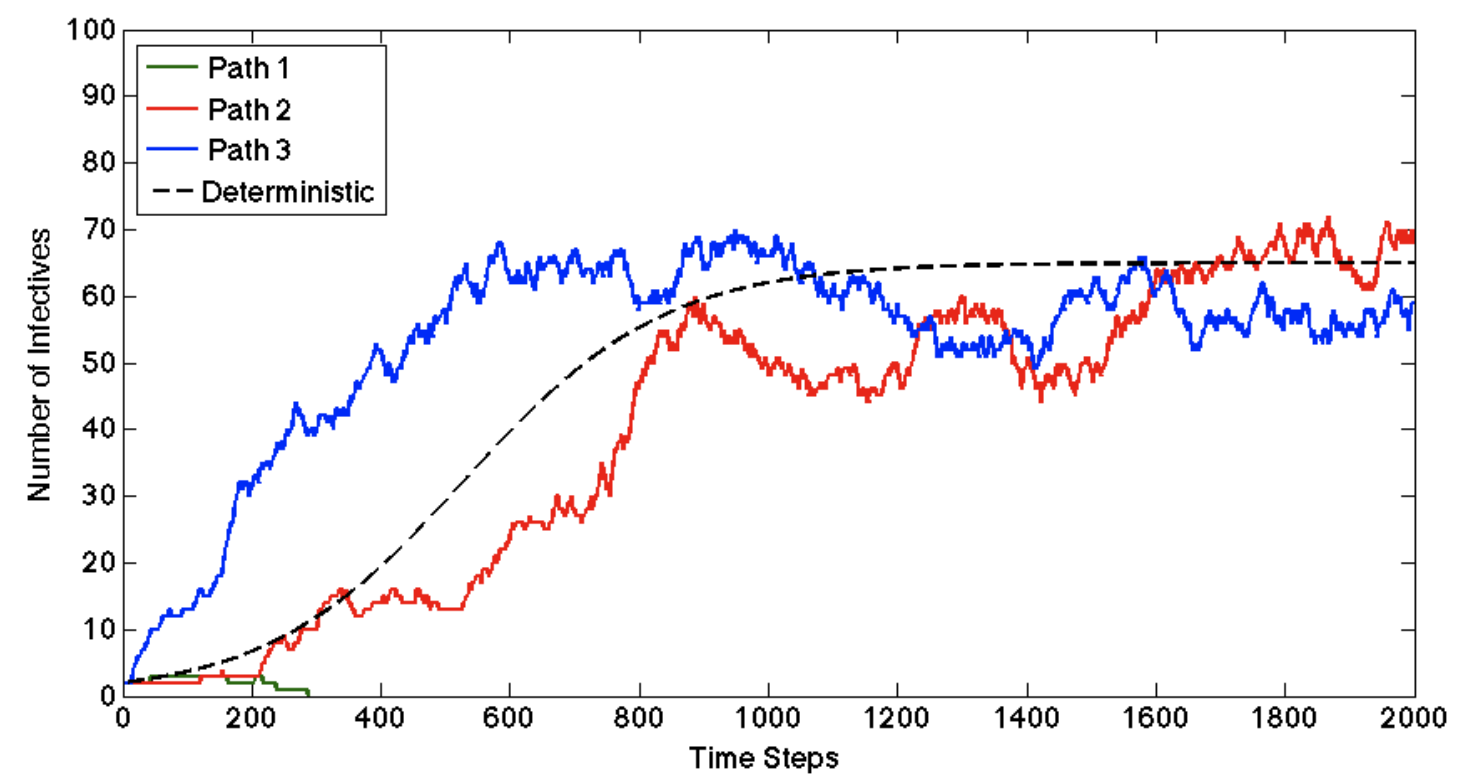

Figure 2.5: Three sample paths of the DTMC SIS model in 2.41. The dashed line shows the deterministic model. The different colours show the different sample paths. For all three sample paths the time step $\Delta t=0.01, N=100, \beta=1, \mu=$ $0.1, \gamma=0.25$.

\subsubsection{The $S I R$ DTMC model}

For the $S I R$ Epidemic Model with vital dynamics, the DTMC model is based on equations 2.34 and 2.35 from section 2.4. The transition probabilities are as follows

$$
p_{(s+k, i+j),(s, i)}(\Delta t)= \begin{cases}\frac{\beta i s}{N} \Delta t & (k, j)=(-1,1) \\ \gamma i \Delta t & (k, j)=(0,-1) \\ \mu i \Delta t & (k, j)=(1,-1) \\ \mu(N-s-i) \Delta t & (k, j)=(1,0) \\ 1-\left[\frac{\beta i s}{N} \Delta t+\gamma i+\mu(N-s)\right] \Delta t & (k, j)=(0,0) \\ 0 & \text { otherwise }\end{cases}
$$

$p_{(s+k, i+j),(s, i)}(\Delta t)$ is the probability that the number of individuals in compartment $S$ changes from $s$ to $s+k$ and the number of individuals in $I$ changes from $i$ to 
$i+j$ over the time step $\Delta t$ We have a constant population size $N$ and birth-rate $=$ death-rate $=\mu$. Because we are assuming all newborns are susceptible, a birth results in an increase in the number of susceptible individuals. Because of the constant population size, a birth also results in a death. If this is an infected individual, we have $(k, j)=(1,-1)$. This has a probability $\mu i \Delta t$ of occurring over one time step. If a birth is balanced by a death among the susceptible population, we would see no change in numbers, i.e. $(k, j)=(0,0)$. If the birth is balanced by the death of a recovered individual, we only see the increase in $S,(k, j)=(1,0)$ [7].

Combining these transition probabilities together, we can get an equation for the probability that $S=s$ and $I=i$ at time $t+\Delta t$.

$$
\begin{array}{r}
p_{(s, i)}(t+\Delta t)=p_{(s+1, i-1),(s, i)}(t) \frac{\beta}{N}(i-1)(s+1) \Delta t+p_{(s, i+1),(s, i)}(t) \gamma(i+1) \Delta t \\
+p_{(s-1, i+1),(s, i)}(t) \mu(i+1) \Delta t+p_{(s-1, i),(s, i)}(t) \mu(N-s+1-i) \Delta t \\
+p_{(s, i),(s, i)}\left(1-\left[\frac{\beta}{N} i s+\gamma i+\mu(N-s)\right] \Delta t\right)
\end{array}
$$

Figure 2.6 shows some sample paths for the SIR DTMC model, along with the curve from the deterministic model. These sample paths were produced in the same way as those for the SIS DTMC model. Because of the added compartment $R$ there are more possible transitions at each time step. In the SIS model a recovery from infection had the same effect as the death of an infected individual, In the $S I R$ stochastic model however, a recovery increases the size of the removed compartment and does not affect the size of $S$. The red sample path dies out near the beginning, resulting in a disease free state early on, this is another example of how a stochastic model may die out despite the predictions made by the deterministic model.

\subsubsection{Continuous Time Markov Chain Models}

The DTMC models described above are defined on discrete time steps, where one individual changes state at each step. The problem with this type of model is that 


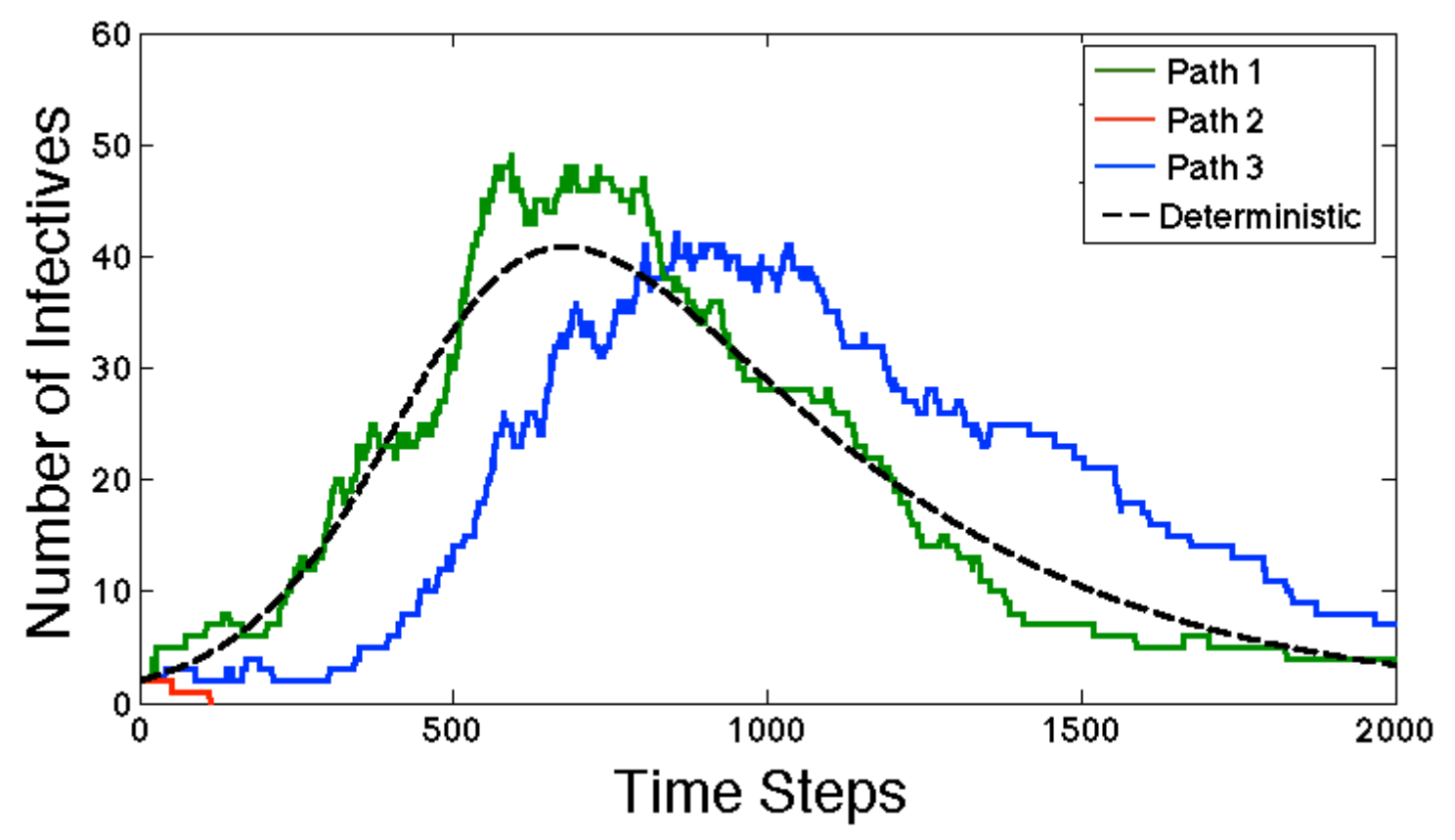

Figure 2.6: Three sample paths of the DTMC SIR model in 2.42, The dashed line shows the deterministic model. The different colours show the different sample paths. For all three sample paths the time step $\Delta t=0.01, N=100, \beta=1, \mu=$ $0, \gamma=0.25$. 
we need to be very careful choosing the size of the time step. Too small a time step and the program takes too long to run, too large a time step and we start to underestimate the change in the population.

One way of overcoming this issue is to use continuous time. A Continuous Time Markov Chain (CTMC) model looks fairly similar to the DTMC model. The transition probabilities are still defined for a small time interval $\Delta t$, but they are referred to as infinitesimal transition probabilities because they are valid for infinitesimally small $\Delta t$. By excluding terms $o(\Delta t)$ in the definitions of the infinitesimal transition probabilities, where $\lim _{\Delta t \rightarrow 0}(o(\Delta t) / \Delta t)=0$, we are able to vary the sizes of the time steps in a exponential distribution and simulate continuous time [7]. For the SIS CTMC model the infinitesimal transition probabilities are defined as follows:

$$
p_{j i}(\Delta t)= \begin{cases}\frac{\beta}{N} i(N-i) \Delta t+o(\Delta t) & j=i+1 \\ (\mu+\gamma) i \Delta t+o(\Delta t) & j=i-1 \\ 1-\left[\frac{\beta}{N} i(N-i)+(\mu+\gamma) i\right] \Delta t+o(\Delta t) & j=i \\ 0+o(\Delta t) & \text { otherwise }\end{cases}
$$

To produce a plot for this model we let $\Delta t=-\log \left(u_{1}\right) / a$, where $u_{1}$ is a uniform random number and $a$ is the probability of change in a time step, $a=1-p_{i, i}(\Delta t)$. We then use another random number $u_{2}$ to decide what type of change occurs in that time step.

Figure 2.7 shows a few of the sample paths produced using the SIS CTMC transition probabilities. Again one of the sample paths shows the disease dying out near the start instead of heading to the endemic equilibrium. While it is possible for a stochastic model to die at from any point in the epidemic, it is usually unlikely. In each model we have shown, the disappearing sample path has died out near the begging of the epidemic when numbers of infectious individuals are low. this shows the vulnerability of the disease to small changes in the initial stages of an epidemic. 


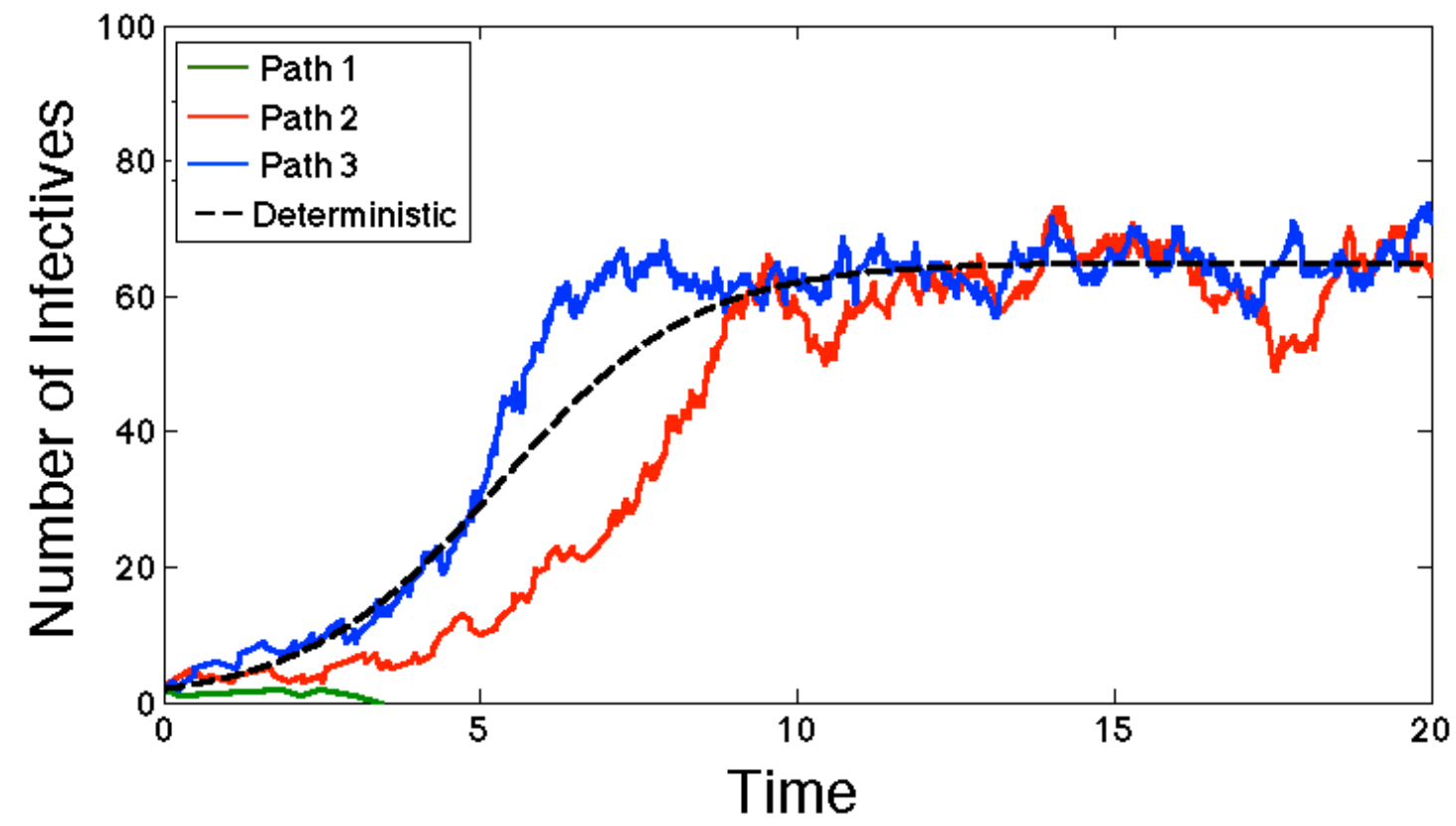

Figure 2.7: Three sample paths of the CTMC SIS model in 2.44. The dashed line shows the deterministic model. The different colours show the different sample paths. For all three sample paths the time step $N=100, \beta=1, \mu=0.1, \gamma=0.25$, 


\section{Chapter 3}

\section{Acute Rheumatic fever}

Acute rheumatic fever is an autoimmune response following an infection by Group A streptococcus bacteria [18]. Group A streptococcus is often presented as strep throat and less often as scarlet fever [1], though there is a recent hypothesis that a skin infection by Group A streptococcus may be responsible for acute rheumatic fever in some communities [11].

If a Group A streptococcus infection is not properly treated, acute rheumatic fever can develop after 2 to 3 weeks [31]. After apparent recovery, the individual experiences an inappropriate immune response. This response is thought to be due to molecular similarity between products of Group A streptococcus degradation and certain human tissues. The heart, joints and central nervous system are the three places this similarity occurs. Acute rheumatic fever is very rare among young children, under the age of 3 [24]. The highest risk coincides with the maturation of the immune system at around ten years of age [31. First time infections of acute rheumatic fever typically occur in children aged between the ages of 5 and 17 [11, 24]. $92 \%$ of cases occur in children under the age of 18 [24].

\subsection{Symptoms and Diagnosis}

Accurate diagnosis of acute rheumatic fever can be an issue and there is currently no definitive laboratory diagnostic test for it [18]. Because of this, diagnosis is 
done clinically with laboratory tests used to support the diagnosis [24].

A patient is usually asymptomatic for about three weeks following the Group A streptococcus infection [1].

Symptoms are described in terms of the Jones criteria, where a combination of major and minor symptoms are used to determine the likelihood that a patient has acute rheumatic fever.

Polyarthritis, affecting the knees elbows, wrists and ankles, accompanied by fever, is the initial warning sign. The hips and spine may also, occasionally, be affected. The arthritis is a major symptom but is only seen in $75 \%$ of cases.

Carditis, another major symptom, usually occurs within three weeks. This is revealed by cardiac sonography in $70 \%$ of cases. Carditis, together with polyarthritis and fever, strongly supports the diagnosis of acute rheumatic fever. Skin signs, such as subcutaneous nodules are rare but may also be observed [24].

Under or over diagnosis in different regions could affect data and recorded incidence in some areas [18]. In the absence of any other explanation however, a diagnosis of rheumatic fever may be given even if symptoms are incomplete. Though if there is abdominal pain initially, the diagnosis of rheumatic fever may be invalidated [24].

\subsection{Susceptibility and Prevention}

Not all individuals infected with Group A streptococcus will develop acute rheumatic fever if untreated. Only certain individuals are susceptible to the disease. $0.3 \%$ to $3 \%$ of a general population will develop acute rheumatic fever following a Group A streptococcus infection [1, 11]. The incidence is much higher among individuals who have had previous attacks of acute rheumatic fever with about $30 \%$ to $80 \%$ having a recurrence of acute rheumatic fever following a Group A streptococcus infection [1]. Interestingly, there is also a higher incidence of acute rheumatic fever among relatives of patients who have, or have had, the disease, when compared with unrelated individuals. It has been noted in past studies that individuals with a family history of acute rheumatic fever are nearly five times more likely to acquire the disease [8]. This suggests a genetic susceptibility to 
acute rheumatic fever [1, 11]. Genetic predisposition is also supported by twin studies where monozygotic twins showed greater similarity in their susceptibility when compared with dizygotic twins, though the lack of greater similarity in susceptibility suggests a complex pattern of inheritance [8]. It also suggests there is a role played by environmental factors in determining susceptibility. There is a long held belief that individuals who are susceptible to acute rheumatic fever also show a hyper reactive immune response to Group A streptococcus antigens [1].

There is usually no increased resistance or susceptibility among particular races or ethnic groups. An outbreak in America in the 1980's however, did occur among middle-class white children with no apparent risk factors aside from possible overcrowding. The degree to which host risk factors are inherited or acquired is unclear [31].

Surveillance involving the collection of epidemiological data is required, to identify 'at risk' groups and help direct control efforts [1].

Recurrence of acute rheumatic fever is relatively common, especially in the 3-5 years following a previous episode [24]. Prevention of recurrent attacks is important, as they can lead to permanent damage to the heart valves. The most cost effective way of preventing rheumatic heart disease is to prevent recurrences of acute rheumatic fever [11]. Regular injections of penicillin (usually monthly), for at least five years following an acute rheumatic fever attack, are used to prevent recurrences. The length of this secondary prophylaxis is dependent on the severity of an individual's previous acute rheumatic fever attacks [11, 31]. Susceptibility to recurrence of acute rheumatic fever is reduced with age [31].

Giving antibiotics to patients with sore throats and other symptoms suggestive of Group A streptococcus remains the only way to prevent primary attacks of acute rheumatic fever. That is besides improving access to medical care and eliminating poverty and overcrowding [11. There has never been a documented case of Group A streptococcus being resistant to penicillin anywhere in the world [4]. Acute rheumatic fever still lacks a vaccine, but if we can learn more about susceptible hosts and how to identify them, prevention of the disease could be greatly simplified [31].

With appropriate health care programs and good medical care, the burden of acute 
rheumatic fever can be reduced [11].

\subsection{Treatment and Recovery}

There is no treatment for acute rheumatic fever. The treatment received by a patient with acute rheumatic fever is purely symptomatic, aside from treatment for heart failure if necessary [19]. The main aim of treatment is to eliminate any remaining Group A streptococcus from the original infection and suppress the inflammation caused by the acute rheumatic fever [10]. Following such treatment, acute rheumatic fever attacks usually cease within 2 months of the initial Group A streptococcus infection. Any relapses after this period are likely to be due to a new infection 32].

\subsection{Group A Streptococcus}

Acute pharyngitis is one of the most common illnesses seen by paediatricians and other primary care physicians [3]. Streptococcus pyogenes strains commonly referred to as Group A Streptococci [1, are the most frequent bacterial cause [12]. It is primarily a childhood disease occurring mostly in children aged between 5 and 15 [12].

Group A Streptococcus is spread through inhalation or contact with secretions from infectious individuals [29]. In temperate climates it usually appears in winter and early spring. During this time of year up to $20 \%$ of asymptomatic children may be streptococcus carriers. These carriers, however, are unlikely to spread the organism to their close contacts and are at low risk of complications such as acute rheumatic fever.

A 'Ping-pong' effect can occur within family groups, where the infection bounces between family or household members as they infect each other and recover. Approximately $25 \%$ of individuals within the household of an index patient may also harbour Group A streptococcus in their upper respiratory tract. There is no credible evidence that family pets may contribute to familial spread. 
Most asymptomatic patients with Group A streptococcus remaining in the upper respiratory tract after a course of antibiotics are streptococcal carriers. While this may mean persistence of the infection in that individual, the circumstances that lead to such asymptomatic carriage usually mean the infection has relatively low virulence. This means asymptomatic carriage has negligible influence in infection models [12]. A small percentage of patients have recurrences of acute pharyngitis associated with Group A streptococcus, shortly after completion of a course of antibiotics [3].

\subsubsection{Symptoms and Diagnosis}

Symptoms of Group A streptococcus infection usually develop after an incubation period of 24 to 72 hours [29]. There is a large overlap between streptococcal and viral pharyngitis in terms of symptoms. Less than half of patients with acute pharyngitis are actually infected with Group A streptococcus. Symptoms include sudden onset of a sore throat, pain on swallowing and fever. Headache, nausea, vomiting and abdominal pain may also be present, especially in children. None of these symptoms however are specific to Group A streptococcus. It can also be difficult to distinguish between a Group A streptococcus carrier currently suffering a non-Streptococcal infection, from a patient with Acute Streptococcal pharyngitis, though the absence of fever or the presence of symptoms such as conjunctivitis, cough, diarrhoea, suggests viral rather than bacterial infection. Because of the overlap in symptoms with viral infections, unless Group A streptococcus can be confidently excluded, a laboratory test should be performed to determine if it is present or not [4]. The most reliable method for detecting the presence of Group A streptococcus in the throat is by culturing a throat swab [12]. Streptococcal pharyngitis is also a self-limiting disease with symptoms disappearing spontaneously within 3-4 days. Rapid identification and treatment of streptococcal pharyngitis can reduce the risk of spread as well as the acute morbidity associated with the disease [4]. 


\subsubsection{Treatment and Recovery}

Effective treatment of a Group A streptococcus infection reduces the risk of acute rheumatic fever by about $90 \%$ [24]. Penicillin, as a treatment for Group A streptococcus, is preventative against acute rheumatic fever [24]. Treatment with penicillin will also reduce the duration of symptoms by 1 or 2 days [29]. Erythromycin is a suitable substitute in the case of patients who are allergic to penicillin [4].

Antimicrobial therapy has no benefit however, in treating pharyngitis that is not caused by Group A streptococcus. Its use in such situations unnecessarily exposes patients to expense and hazards as well as possibly contributing to the emergence of antibiotic-resistant bacteria. It has been shown that therapy can be postponed for up to 9 days after symptoms first appear, and still safely prevent the onset of acute rheumatic fever [4]. An untreated Group A streptococcus infection will last for 7 to 10 days [29]. In about $10 \%$ of cases, Group A streptococcus will remain present in the throat even after adequate treatment [24]. For patients whose symptomatic episodes do not decrease in frequency over time, without alternative explanation, surgical removal of the tonsils may be considered. Throat cultures should be performed regularly for patients with a history of acute rheumatic fever [4].

Vaccines for Group A streptococcus are currently being investigated. One important issue with the vaccine is that it does not itself, induce the acute rheumatic fever it is intended to prevent [12].

\subsubsection{Rheumatogenic Strains}

Infection of the throat, by Group A streptococcus, is usually required for acute rheumatic fever to be a risk [24]. Studies have shown Group A streptococcus strains that colonise the skin are rarely associated with cases of acute rheumatic fever [31]. Not all infections due to Group A streptococcus can result in acute rheumatic fever. Those that do are considered extremely virulent and the attack rate of the resultant acute rheumatic fever is dependent on the host's immune response [31]. The onset of acute rheumatic fever in an individual may be the 
result of an increase of virulence in a specific strain of Group A streptococcus [24]. Identifying the particular strains that lead to acute rheumatic fever can be difficult however, as often patients do not remember having a sore throat. By the time acute rheumatic fever is diagnosed, the infecting strain is often gone [31]. While the separation of skin and throat strains of Group A streptococcus is fairly simple, the differences between throat strains associated with acute rheumatic fever and those that are not is not clear other than their differing levels of virulence [31].

There appears to be some seasonal variation, such that throat colonising Group A streptococcus is not often associated with acute rheumatic fever during the summer. It is during the winter that acute rheumatic fever follows Group A streptococcus [31].

Overall the development of acute rheumatic fever and its severity is thought to depend on the genetic susceptibility of an individual, the virulence of the Group A streptococcus strain, environmental factors and an abnormal immune response [8, 11].

\subsection{New Zealand Infection Rates}

In a study conducted by Jaine et.al. annual rates of first time acute rheumatic fever cases in New Zealand between 1996 and 2005 were found to be 3.4 per 100 000. This is an average of 125 first time acute rheumatic fever hospital admissions per year [18].

There also appears to be a strong ethnic association with acute rheumatic fever in New Zealand, making ethnicity an important risk factor. While New Zealand European and other ethnicities have seen a significant drop in acute rheumatic fever rates, there have been increases among Maori and Pacific peoples such that overall rates in New Zealand have not dropped since the 1980's. The highest estimated incidence of acute rheumatic fever among school children is in SubSaharan Africa, but the highest published incidence in recent times is among the indigenous populations of Australia and New Zealand [11]. Rates among Maori are approximately 22 times that of New Zealand Europeans, and Pacific peoples 


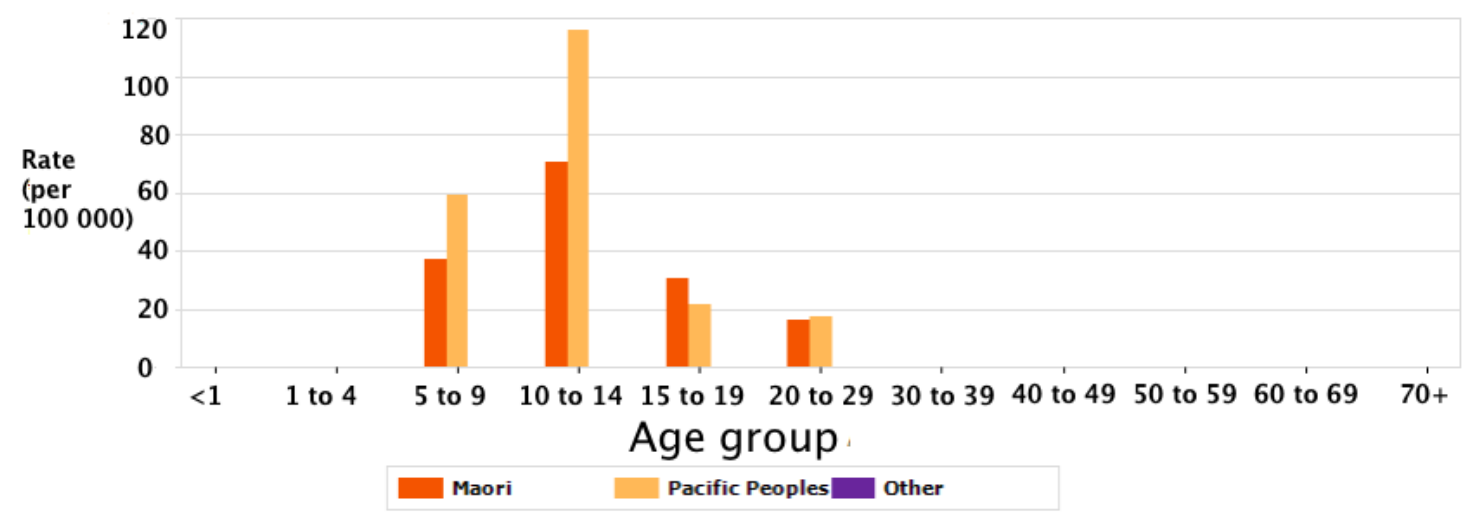

Figure 3.1: Rheumatic fever rates for 2010 by age group and ethnicity. Rates are per 100000 population. This was sourced from the New Zealand public health observatory website, Notifiable disease page. The numbers of acute rheumatic fever amoung the other ethnicities do not show up here because they are too small, they represent only 6 cases in total or 0.000076 per 100000 individuals.

have a rate 75 times greater [18]. Figure 3.1 shows the rates for different ethnicities and age groups in 2010 .

As the graph in Figure 3.1 shows, acute rheumatic fever is a huge issue among Maori and Pacific peoples, while barely affecting any other ethnicities. In 2010 there were 104 cases among Maori, 58 among Pacific peoples and 6 cases among all the other ethnicities together.

In New Zealand recurrences are again, more likely to occur among the Maori and Pacific peoples, when compared with the rest of the population, accounting for well over $90 \%$ of recurrent cases. Overall recurrence rates in New Zealand are low, with an average of only six cases per year for the years 1996 to 2005 [18].

New Zealand continues to maintain a high rate of acute rheumatic fever for a developed country. Table 3.1 gives rates of acute rheumatic fever from around the world [9]. We can easily see how much more prevalent acute rheumatic fever is among the maori, aboriginal and pacific island populations comapared with other ethnicities and regional populations.

Figure 3.2 shows the number of acute rheumatic fever cases seen by health professionals in New Zealand each month since 1997. 


\begin{tabular}{|l|c|c|}
\hline Region & Median incidence* & Annual No. cases \\
\hline Sub-Saharan Africa & 13.4 & 24000 \\
\hline South central Asia & 54.0 & 184000 \\
\hline Asia other & 21.2 & 26500 \\
\hline Latin America & 19.6 & 21000 \\
\hline Middle east and north Africa & 13.4 & 11000 \\
\hline Eastern Europe & 10.2 & 4000 \\
\hline Pacific \& indigenous Australia/NZ & 374.0 & 8000 \\
\hline Established market economies** & 10.0 & 11000 \\
\hline China & 21.2 & 47000 \\
\hline Total aged 5-14 years &.. & 336500 \\
\hline Total all ages &.. & 471000 \\
\hline
\end{tabular}

* cases per 100,000.

** Non-indigenous Australia and New Zealand, Western Europe,

Northern Europe, Southern Europe, Northern America and Japan

$\dagger$ Assumes $40 \%$ of all acute rheumatic fever cases occur over 14 years of age.

Table 3.1: Estimated annual number of acute rheumatic fever cases in children aged 5-14 years, and extrapolation to all ages [9].

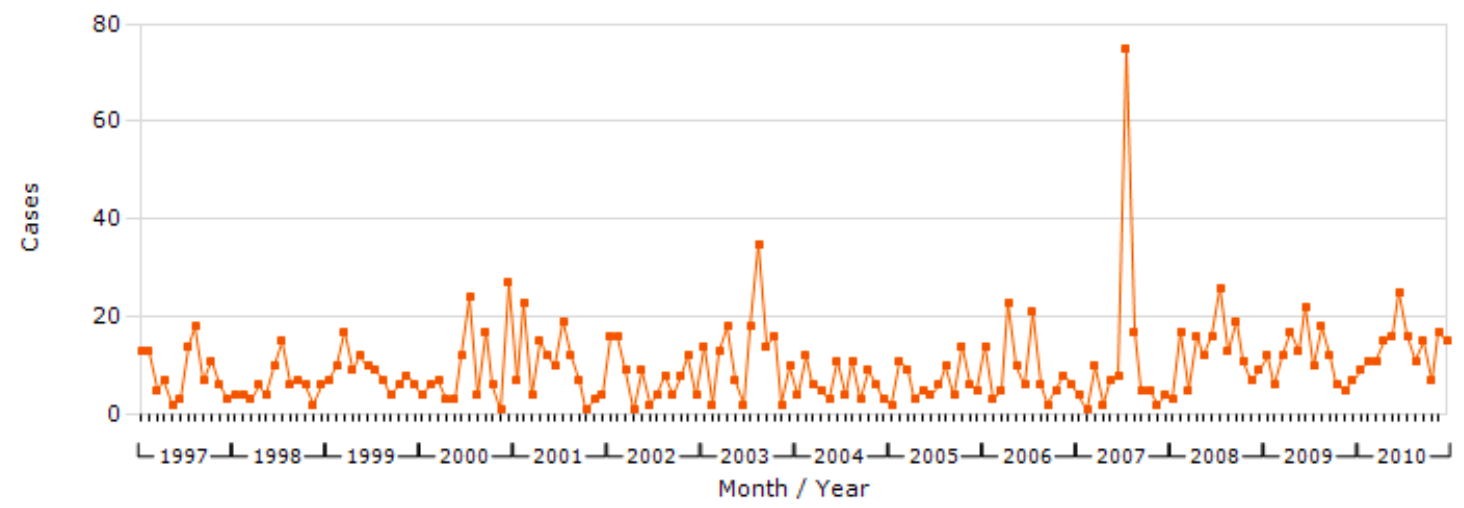

Figure 3.2: Rheumatic fever cases by month since 1997. This was sourced from the New Zealand public health observatory website, Notifiable disease page. 


\section{Total Rheumatic fever by month 1997 to 2009}

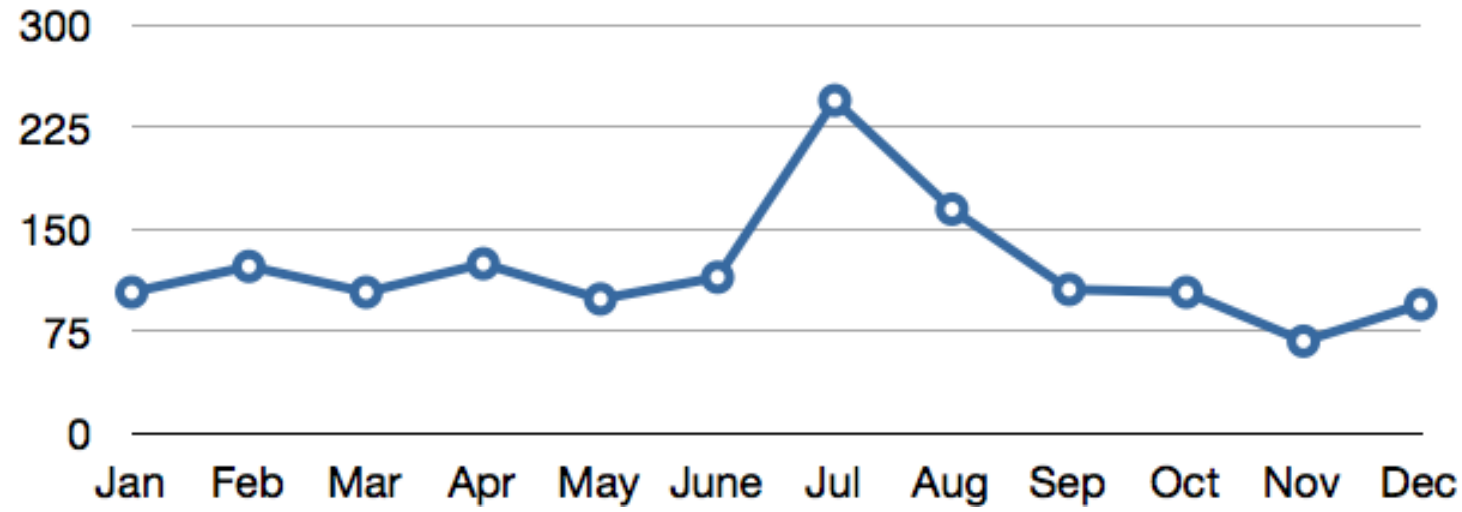

Figure 3.3: Rheumatic fever cases by month since 1997 summed together. Data were sourced from the New Zealand public health observatory website, Notifiable disease page.

Geographically incidence is highest in the upper North Island with low incidence in the South Island. This geographic variation is strongly correlated with ethnic distribution in New Zealand.

Acute rheumatic fever is a notifiable disease in New Zealand [18]. This means medical practitioners are required to notify local medical health officers of any acute rheumatic fever diagnoses they make. Failure to do so is an offence against the health act [22]. The numbers of reported cases, between 1996 and 2005, peak around early winter for the months June - August. The lowest incidence was recorded in October, November and December [18]. Figure 3.3 shows the monthly pattern a bit more clearly.

These patterns in age, ethnicity and season give us some things to focus on in developing a model for disease incidence. 


\section{Chapter 4}

\section{A Basic Model for Rheumatic Fever}

\subsection{Building the Basic Model}

As we saw in Chapter 3 , acute rheumatic fever is an autoimmune response to infection by Group A streptococcus. This means individuals are moving from being infected to developing acute rheumatic fever. If we label the infected compartment $I$ and the compartment containing those with acute rheumatic fever as $A$, individuals flow from $I$ to $A$, as shown in Figure 4.1, at the rate they develop acute rheumatic fever.

The infected individuals must come from somewhere, so we need a compartment that contains those individuals susceptible to infection. This is the Susceptible compartment which we will label $S$. As Figure 4.1 shows, susceptible individuals move from $S$ to $I$ as they become infected and from there move on to $A$.

Because Group A streptococcus is an illness from which no immunity is retained upon recovery, we need an SIS type model. All individuals move back to the susceptible compartment as they recover. Individuals may recover from either the $I$ or $A$ compartment as not everyone develops acute rheumatic fever after having a Group A streptococcus infection. Figure 4.1 shows the flow of individuals once 


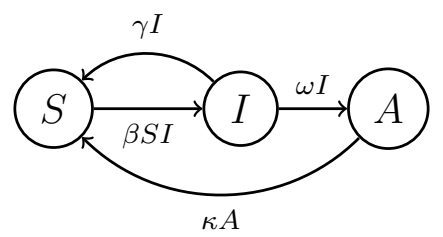

Figure 4.1: Flow chart for the SIAS epidemic model, given by 4.1, showing the rate of movement between compartments as individuals move from Susceptible, though Infectious to developing Acute rheumatic fever, and the different paths they can take back to the Susceptible compartment.

we add in recovery.

We will refer to this as the SIAS model. The differential equations for this model are shown in 4.1 below.

$$
\begin{aligned}
& \frac{d S}{d t}=-\beta S I+\gamma I+\kappa A \\
& \frac{d I}{d t}=\beta S I-\gamma I-\omega I \\
& \frac{d A}{d t}=\omega I-\kappa A
\end{aligned}
$$

The rate of new infections is proportional to both the number of susceptible individuals and the number of infectious individuals. $\beta$ is the proportionality constant and represents the rate of contact resulting in infection. $\gamma$ is the rate of recovery of infected individuals back into the susceptible population. $\omega$ is the rate at which infected individuals develop acute rheumatic fever and $\kappa$ is the rate at which they recover. Figure 4.1 shows the flow of individuals and the rates at which they move between compartments in this basic model.

\subsubsection{Assumptions}

For this model we are assuming individuals with acute rheumatic fever are no longer infectious but cannot be infected either. Though individuals with acute rheumatic fever may still retain some bacteria, this is minimal and often gone by the time they are diagnosed [31], and due do the immune response resulting in acute rheumatic fever they are unlikely to contract a new infection while in 
compartment $A$. We are also assuming we have a homogeneous population where individuals mix evenly and each individual has the same chance of being infected and developing acute rheumatic fever.

$N=S+I+A$ is the total population size. In this model we are assuming the population size is constant, therefore $\frac{d S}{d t}+\frac{d I}{d t}+\frac{d A}{d t}=0$. This means we can use $A=N-S-I$ to substitute for $A$. The acute rheumatic fever compartment works a bit like an $R$ class in an $S I R$ type model, it contains individuals who were infected and are temporarily immune to reinfection. While $A$ does have an effect on the dynamics of $S$ and $I$, we can easily find $A$ from $S, I$ and $N$ at any point. Substituting $A=N-S-I$ into 4.1 gives us the reduced system

$$
\begin{aligned}
& \frac{d S}{d t}=-\beta S I+\gamma I+\kappa(N-S-I) \\
& \frac{d I}{d t}=\beta S I-\gamma I-\omega I
\end{aligned}
$$

\subsection{Equilibrium Points for the $S I A S$ Model}

We find the equilibrium points in the same way we found them for the $S I S$ and $S I R$ models in section 2.2.3. First set the righthand side of each equation equal to zero, Then solve for $S$ and $I$. The second equation from 4.2 gives us

$$
\begin{aligned}
\beta S I & =\gamma I+\omega I \\
& \Rightarrow I=0 \text { or } \beta S=\gamma+\omega
\end{aligned}
$$

If $I=0$ the first equation of 4.2 gives us

$$
\begin{gathered}
\kappa N=\kappa S \\
N=S
\end{gathered}
$$

$\kappa$ is the constant rate of recovery from acute rheumatic fever and non-zero. So the first equilibrium point is at $(N, 0)$. This is the disease free equilibrium.

If $I \neq 0$ then we have $\beta S=\gamma+\omega$. By substituting this into the first equation of 
4.2 we get

$$
\begin{aligned}
-I(\gamma+\omega)+\gamma I+\kappa N-\frac{\kappa(\gamma+\omega)}{\beta}-\kappa I & =0 \\
-I(\omega+\kappa)+\kappa\left(N-\frac{\gamma+\omega}{\beta}\right) & =0 \\
I(\omega+\kappa) & =\kappa\left(N-\frac{\gamma+\omega}{\beta}\right) \\
I & =\frac{\kappa(\beta N-\gamma-\omega)}{\beta(\omega+\kappa)}
\end{aligned}
$$

giving us the endemic equilibrium point at

$$
(S, I)=\left(\frac{\gamma+\omega}{\beta}, \frac{\kappa(\beta N-\gamma-\omega)}{\beta(\omega+\kappa)}\right)
$$

\subsubsection{Stability of the Equilibrium Points}

To determine the stability of each equilibrium point we find the Jacobian matrix for 4.2

$$
\left[\begin{array}{cc}
-\beta I-\kappa & -\beta S+\gamma-\kappa \\
\beta I & \beta S-\gamma-\omega
\end{array}\right]
$$

At the disease free equilibrium $(N, 0)$ this becomes

$$
\left[\begin{array}{cc}
-\kappa & -\beta N+\gamma-\kappa \\
0 & \beta N-\gamma-\omega
\end{array}\right]
$$

which has two eigenvalues, $-\kappa$ and $\beta N-\gamma-\omega$. Because $-\kappa<0$ always, the disease free equilibrium is stable if $\beta N<\gamma+\omega$ and unstable if $\beta N>\gamma+\omega$. This makes sense because if the initial rate of infection, $\beta N$, is less than the rate at which individuals leave the infectious compartment, $\gamma+\omega$, then the infectious $I$ will shrink faster than it grows until the disease dies out in the population.

At the endemic equilibrium $(S, I)=\left(\frac{\gamma+\omega}{\beta}, \frac{\kappa(\beta N-\gamma-\omega)}{\beta(\omega+\kappa)}\right)$ we get the Jaco- 
bian matrix

$$
\left[\begin{array}{cc}
\frac{-\kappa(\beta N-\gamma+\kappa)}{\omega+\kappa} & -\omega-\kappa \\
\frac{\kappa(\beta N-\gamma-\omega)}{\omega+\kappa} & 0
\end{array}\right]
$$

Fixed points are stable if the determinant of the Jacobian matrix is positive and the trace is negative. The determinant of the Jacobian matrix 4.6 is

$$
(\omega+\kappa) \times \frac{\kappa(\beta N-\gamma-\omega)}{\omega+\kappa}=\kappa(\beta N-\gamma-\omega)
$$

Which is positive if $\beta N>\gamma+\omega$.

The trace of this matrix is

$$
\frac{-\kappa(\beta N-\gamma+\kappa)}{\omega+\kappa}
$$

Which is negative if $\beta N>\gamma-\kappa$. For $\kappa>0$,

$$
\gamma-\kappa<\gamma<\gamma+\omega
$$

So this equilibrium point is stable as long as $\beta N>\gamma+\omega$.

This is similar to what we saw in Section 2.2.3.1 with the endemic equilibrium for the SIS model. The condition of stability of this equilibrium point is also a condition for the valid existence of this equilibrium point. At the endemic equilibrium $\beta S=\gamma+\omega$, so $\beta N$ must be greater than $\beta S$, as $N=S+I+A$ and to be biologically realistic we cannot have a negative number of individuals in any compartment. This means $\beta N>\beta S=\gamma+\omega$ for this equilibrium point to realistically exist. That is, when the equilibrium is achievable it is also stable. The change of stability about the point $\beta N=\gamma+\omega$, is again an example of transcritical bifurcation.

To find the nature of $\mathrm{A}$ and its value at each equilibrium point we can use $N=$ $S+I+A$ and substitute the appropriate values into 4.1. Note that $A$ is also stationary at the equilibrium points since $A=N-S-I$ and $S$ and $I$ are stationary. So at $(N, 0), A=0$.

This makes sense as when $S=N$ everyone is in the susceptible compartment. There are no individuals with acute rheumatic fever or in the infected compartment 


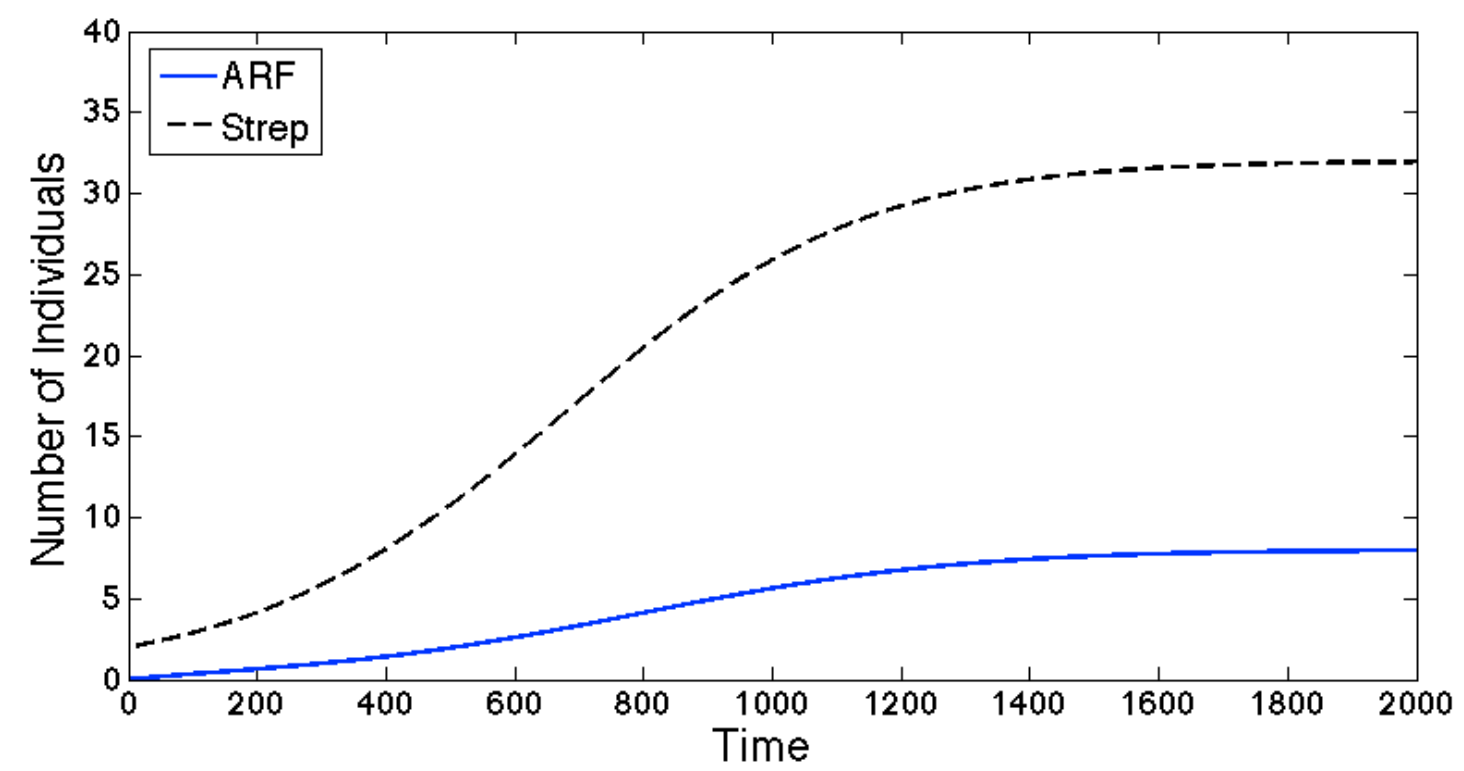

Figure 4.2: A plot of a numerical solution to the $S I A S$ model 4.1. with $N=$ $100, \beta=0.01, \gamma=0.4, \omega=0.2, \kappa=0.8$ and 2 initial infectious individuals. Under these conditions the numbers of Group A streptococcus (Strep $=I$ ) and acute rheumatic fever $(\mathrm{ARF}=A)$ head to an equilibrium at 32 and 8 respectively

to be at risk of developing acute rheumatic fever.

At the endemic equilibrium

$$
A=\frac{\omega(\beta N-\gamma-\omega)}{\beta(\omega+\kappa)}=\frac{\omega I}{\kappa} .
$$

$A$ is directly proportional to $I$. This is also clear from the third equation in 4.1 .

$$
\frac{d A}{d t}=0 \Longrightarrow \omega I=\kappa A
$$




\subsection{Triangle of Validity}

Because $N=S+I+A$ is constant we can 'decouple' $A$ from the system of equations as was done in 4.2 . We cannot drop it entirely however. Aside from $A$ being the compartment we are interested in in the first place, it also has a large influence on the values of $I$ and $S$ and their valid existence.

The endemic equilibrium point is at $\beta S=\gamma+\omega . S \leq N$ always, so for the endemic equilibrium to have valid values of $S$ and $I$, we require $\beta N>\gamma+\omega$. The disease free equilibrium is at $S=N$ and is stable as long as $\beta S=\beta N<\gamma+\omega$. This puts the endemic equilibrium outside the 'triangle of validity', or valid range of possible values for $S$ and $I$, as $\beta S=\gamma+\omega>\beta N$ is an unrealistic scenario.

If we plot $I$ against $S$ and join the points $(N-A, 0)$ and $(0, N-A)$ to represent the boundary above which points are no longer valid, we get a triangle. Figure 4.3 shows this along with a numerical solution of 4.1 with infectious individuals plotted against the number of susceptibles.

So for the endemic equilibrium to be valid the disease free equilibrium must lose its stability. It also means that when the endemic equilibrium has valid values, it has a negative trace and positive determinant. So when it is valid, the endemic equilibrium is always asymptotically stable. Under the conditions at which the endemic equilibrium is unstable it is outside of the 'triangle of validity'.

This change of stability about the point $\beta N=\gamma+\omega=\beta S$ is an example of transcritical bifurcation [33]. In this case either the disease free equilibrium is always valid, but is unstable when the endemic equilibrium exists. The endemic equilibrium only exists when the disease free equilibrium is unstable, the endemic equilibrium is always stable when it exists.

We saw this same, transcritial bifurcation, behaviour in our analysis of the SIS model in section 2.2.3.1.

Theoretically the same triangle of validity, seen in our SIAS model can apply to the $S I R$ model. The problem is that there is no feedback into $\mathrm{S}$ from either $I$ or $R$ so $R$ gets bigger and the triangle decreases in size until $I=0$. 


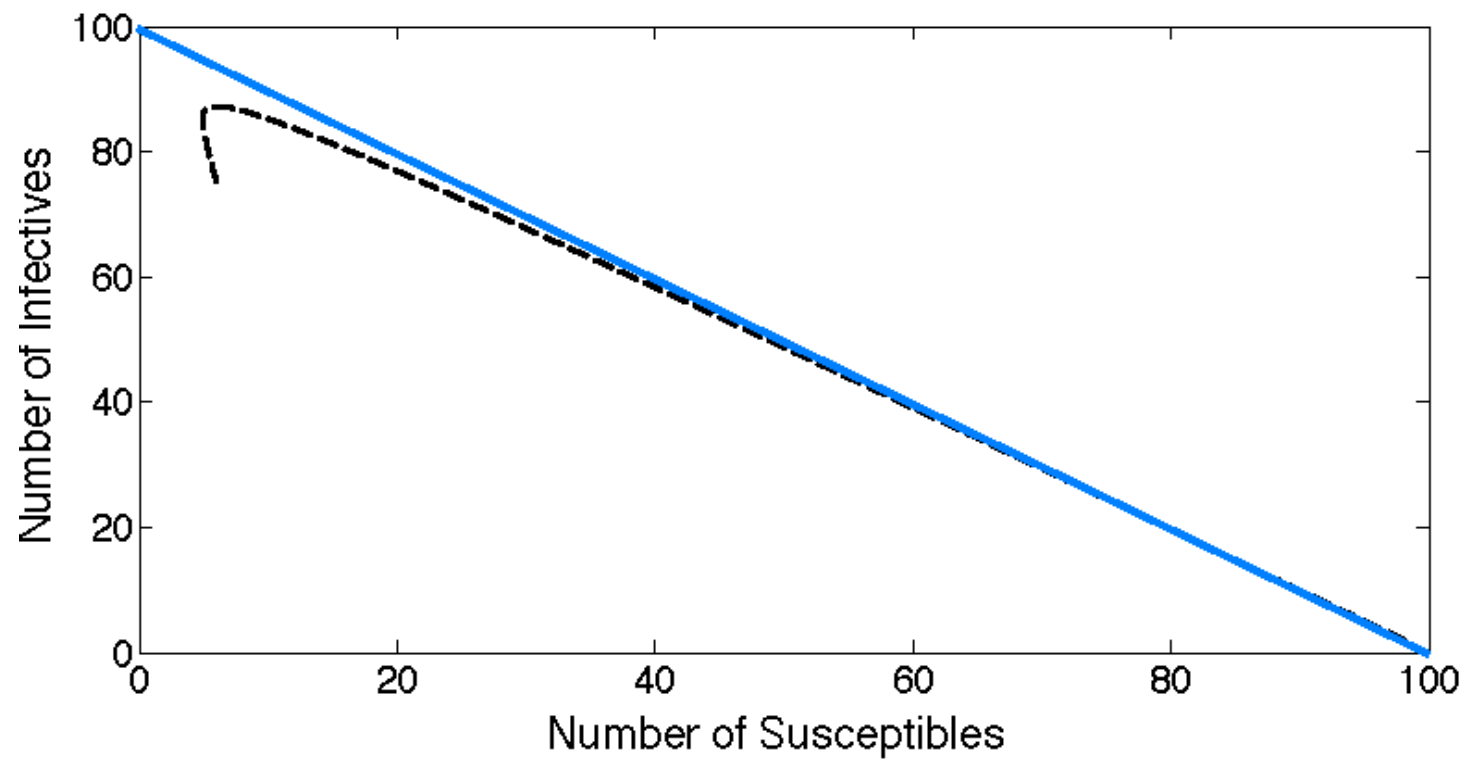

Figure 4.3: A plot of $I$ against $S$ for $S I A S$ model. $N=100, \beta=0.1, \gamma=$ $0.4, \omega=0.2, \kappa=0.8$ with 2 initial infectious individuals. The line curves down before we reach $S=0$ as this can only happen if $\beta \longrightarrow \infty$ or if $\gamma+\omega=0$, i.e. we have no recovery. The solid blue line shows the direct line between $(S, I)=(0, N)$ and $(S, I)=(N, 0)$ 


\subsection{1 $\mathcal{R}_{0}$ for the Basic $S I A S$ Model}

In section 2.2, equation 2.13 , we defined the basic reproduction number $\mathcal{R}_{0}$ for the $S I R$ model as

$$
\mathcal{R}_{0}=\frac{\beta N}{\gamma}
$$

This is the rate of infection of susceptible individuals in a fully susceptible population, (ie. when $S=N$ ) divided by the rate at which individuals leave the infectious compartment [7]. For our acute rheumatic fever model this would mean

$$
\mathcal{R}_{0}=\frac{\beta N}{\gamma+\omega}
$$

It is instructive to relate $\mathcal{R}_{0}$ to the stability of the equilibrium points. When the endemic equilibrium is valid $\beta N>\gamma+\omega$ and $\mathcal{R}_{0}>1$, meaning more than one susceptible individual is infected by each infected individuals, which would imply an epidemic is likely to occur. It makes sense that this threshold is reached simultaneously with the appearance of a stable endemic equilibrium.

\subsection{A Basic Stochastic Model}

Using our basic acute rheumatic fever model, we can derive a set of transition properties similar to what was done for the $S I R$ model in equation 2.42 and the $S I S$ model in section 2.5.2. Using $S=N-I-A$ we can show the probability of a single change of state. This is the probability that one individual changes compartment.

\subsubsection{Discrete Time Markov Chain Model}

$p_{(i+j, a+k),(i, a)}(\Delta t)$ is the probability that the number of individuals in the infectious compartment will change from $I(t)=i$ to $I(t+\Delta t)=i+j$ and the number of individuals with acute rheumatic fever will change from $A(t)=a$ to $A(t+\Delta t)=$ $a+k$ over the time step $\Delta t$. The transition probabilities for the SIAS DTMC 


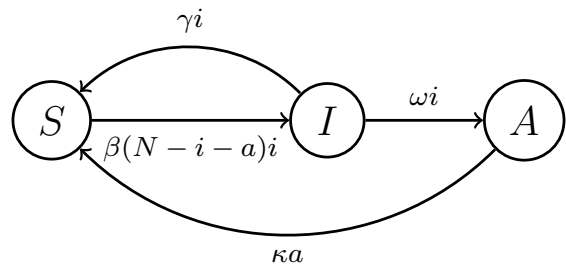

Figure 4.4: Flow chart for the SIAS DTMC model showing the transition probabilities for moving between each compartment.

model are

$$
p_{(i+j, a+k),(i, a)}(\Delta t)= \begin{cases}\beta i(N-i-a) \Delta t & (j, k)=(1,0) \\ \gamma i \Delta t & (j, k)=(-1,0) \\ \omega i \Delta t & (j, k)=(-1,1) \\ \kappa a \Delta t & (j, k)=(0,-1) \\ 1-[\beta i(N-i-a)+i(\gamma+\omega)+\kappa a] \Delta t & (j, k)=(0,0) \\ 0 & \text { otherwise }\end{cases}
$$

These transition probabilities are derived from the differential equations 4.1 seen earlier in the chapter.

The probability that $I=i$ and $A=a$ at time $t+\Delta t$ in terms of the transition probabilities is given by

$$
\begin{aligned}
p_{(i, a)}(t+\Delta t) & =p_{(i-1, a)}(t) \beta(i-1)(N-i+1-a) \Delta t \\
& +p_{(i+1, a)}(t)(\gamma)(i+t) \Delta t+p_{(i+1, a-1)}(t) \omega(i+1) \Delta t \\
& +p_{(i, a+1)}(t)(\kappa)(a+1) \Delta t \\
& +p_{(i, a)}(t)(1-[\beta i(N-i-a)+i(\gamma+\omega)+a(\kappa)] \Delta t)
\end{aligned}
$$

Figure 4.5 shows two sample paths for the transition probabilities in 4.9 . The plots for both Group A streptococcus $(I)$ and acute rheumatic fever $(A)$ are shown along with their deterministic plots. 


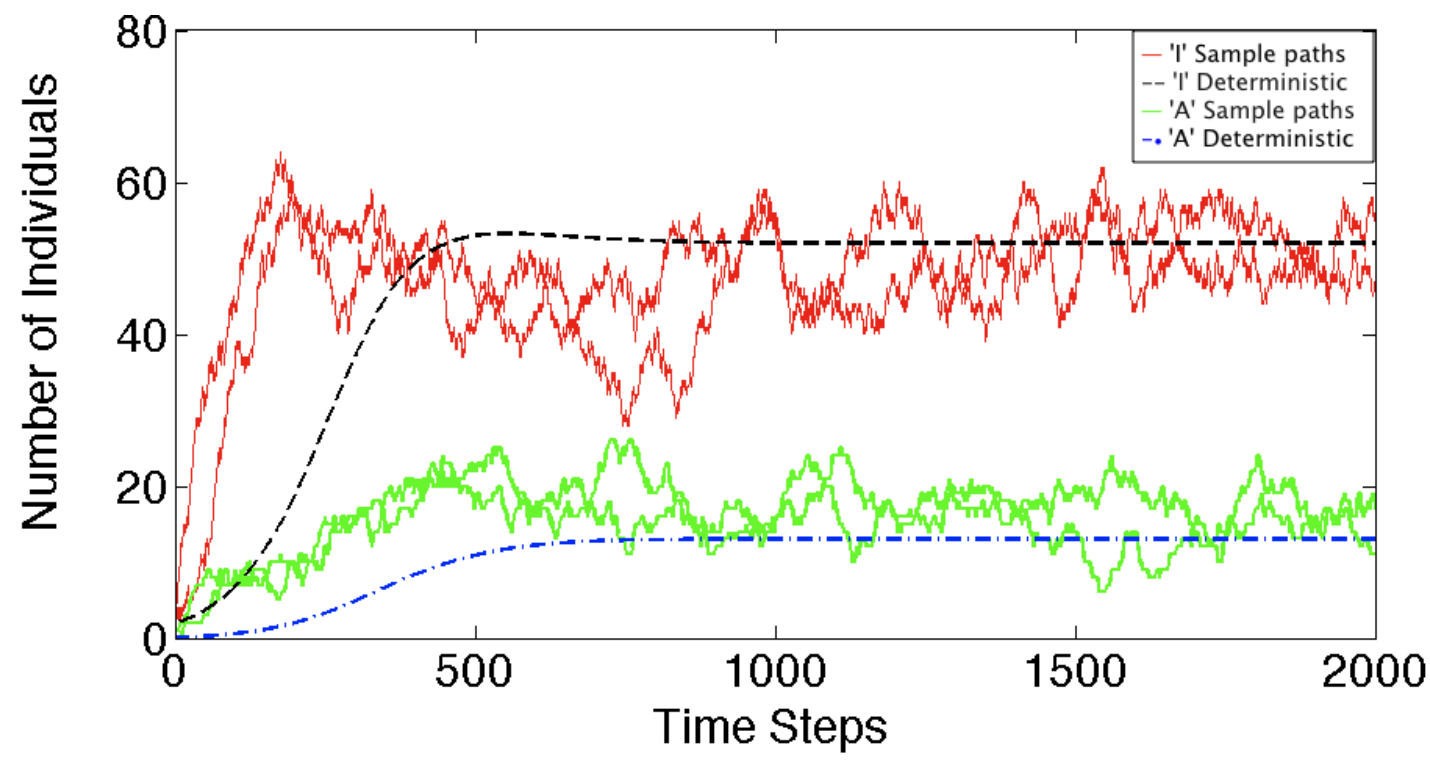

Figure 4.5: Two sample paths of the DTMC SIAS model given in 4.9. The solid lines are the DTMC sample paths. The dashed lines show the deterministic model. The lower set of lines is the number of individuals with acute rheumatic fever $(A)$ and the higher are the number with Group A streptococcus $(I) . N=100, \beta=$ $0.002, \gamma=0.5 . \omega=0.2, \kappa=0.8$ 


\subsubsection{Continuous Time Markov Chain Model}

As we said earlier in section 2.5.2, by using a Continuous Time Markov Chain (CTMC) model, we allow changes to occur over time steps of varying size. A CTMC model allows us to more accurately represent changes taking place in continuous time. Because of this increased accuracy, from now on we will only be using continuous time in our stochastic models.

For the basic $S I A S$ model we can define the CTMC infinitesimal probabilities as follows

$$
\begin{aligned}
& p_{(i+j, a+k),(i, a)}(\Delta t)= \\
& \begin{cases}\frac{\beta i(N-i-a)}{N} \Delta t+o(\Delta t) & (j, k)=(1,0) \\
\gamma i \Delta t+o(\Delta t) & (j, k)=(-1,0) \\
\omega i \Delta t+o(\Delta t) & (j, k)=(-1,1) \\
\kappa a \Delta t+o(\Delta t) & (j, k)=(0,-1) \\
1-\left[\frac{\beta i(N-i-a)}{N}+i(\gamma+\omega)+\kappa a\right] \Delta t+o(\Delta t) & (j, k)=(0,0) \\
o(\Delta t) & \text { otherwise }\end{cases}
\end{aligned}
$$

Figure 4.6 shows a plot of two sample paths for the SIAS CTMC infinitesimal transition probabilities. Comparing them with the deterministic plot from the equations in 4.1 we can see that the sample paths generally follow the deterministic curve but with some variation induced by the random choice made at each time step. 


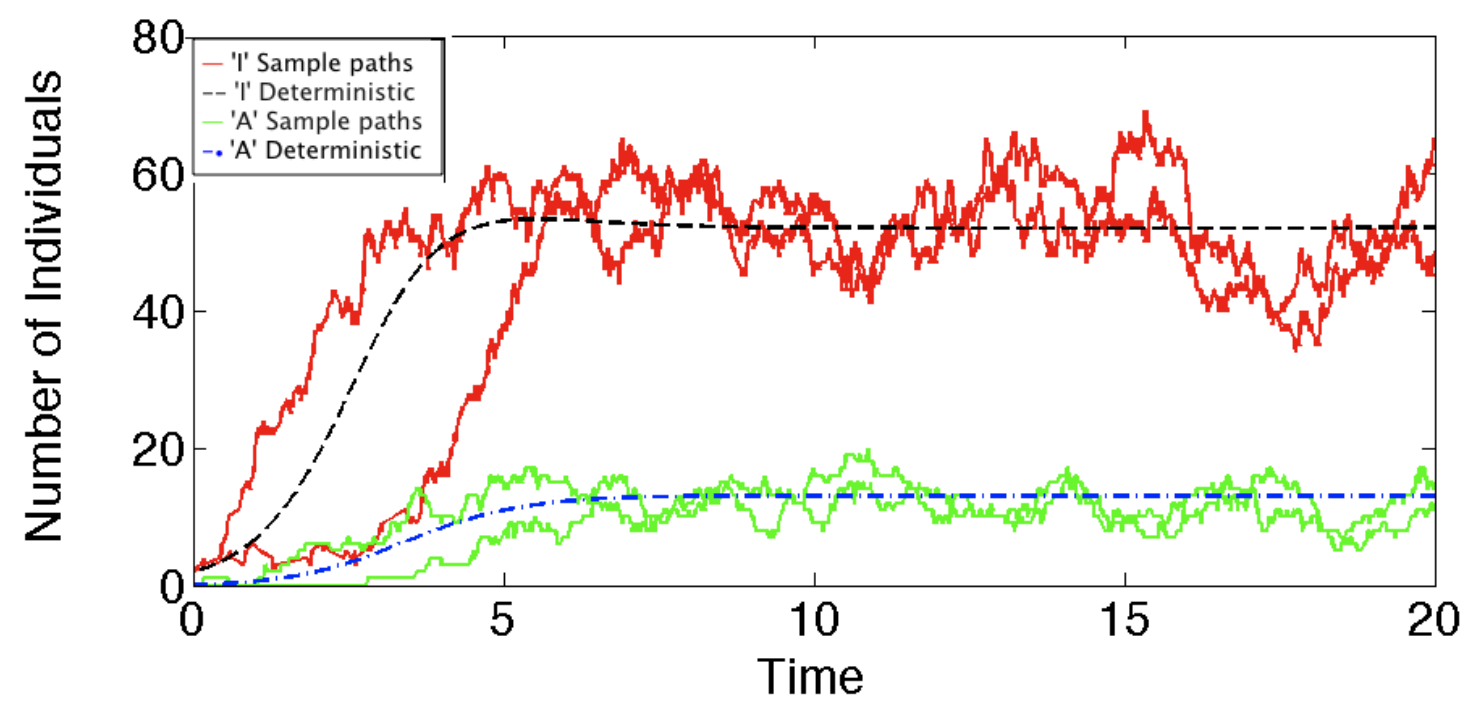

Figure 4.6: Two sample paths of the CTMC SIAS model seen in 4.11. The solid lines are the CTMC sample paths. The dashed lines show the deterministic model. The lower set of lines is the number of individuals with acute rheumatic fever $(A)$ and the higher are the number with Group A streptococcus $(I) . N=100, \beta=$ $0.02, \gamma=0.5 . \omega=0.2, \kappa=0.8$ 


\section{Chapter 5}

\section{Developing the Model Further}

\subsection{Vital Dynamics}

Because there is no immunity conferred by Group A streptococcus, if we want to include the possibility that people may die from acute rheumatic fever, we must also include natural birth and death rates. Otherwise our whole population would eventually die out from the disease.

If people die from acute rheumatic fever, there will also be a fraction who recover. Let $f$ be this fraction that recover, so $1-f$ of individuals who develop acute rheumatic fever will die. In this model the population size will not always be constant, so $N$ is also changing. Our system of equations for acute rheumatic fever with vital dynamics is given in 5.1 below:

$$
\begin{aligned}
& \frac{d S}{d t}=b N-\beta S I+\gamma I+\kappa f A-\mu S \\
& \frac{d I}{d t}=\beta S I-\gamma I-\omega I-\mu I \\
& \frac{d A}{d t}=\omega I-\kappa A-\mu A
\end{aligned}
$$

We are assuming no vertical transmission of Group A streptococcus from mother to newborn, so all newborns are born into the susceptible compartment. $b$ is the birthrate in the population. $\mu$ is the natural death rate that would happen in the 


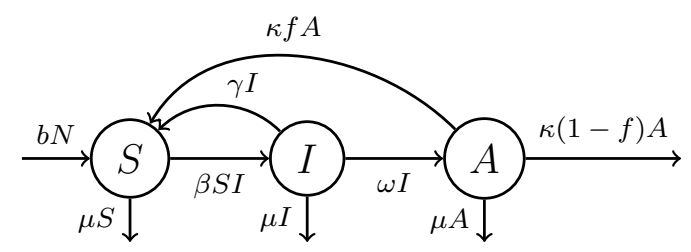

Figure 5.1: Flow chart for the SIAS epidemic model with vital dynamics given by 5.1 .

absence of disease. We should note that, since $N=S+I+A$ we can define

$$
\frac{d N}{d t}=b N-(1-f) \kappa A-\mu N
$$

Apart from the addition of a death rate the equations for $I$ and $A$ have not changed from 4.1. The equation for $S$ gains birth and death rates and $\kappa f A$ replaces $\kappa A$ as only a fraction $f$ survive having acute rheumatic fever.

\subsubsection{Equilibria}

$\frac{d N}{d t}=0 \Longrightarrow(b-\mu) N-(1-f) \kappa A=0$. This means the only way $N$ can reach an equilibrium where $\frac{d N}{d t}=0$ is if $b=\mu$ and $f=1$. There are two ways we could deal with this problem. We could replace $b N$ with $b(N)$ where $b(N)$ is logistic, so as the population size increases the birth-rate approaches the death-rate so the population size approaches an equilibrium, also known as the carrying capacity. So as $N$ approaches this carrying capacity, $b(N)$ approaches $\mu N$. While this method is useful it complicates the system a bit more.

When dealing with the smaller periods of time usually associated with epidemics we can use the fact that the population will not normally vary in size much. In this case we can say $b \approx \mu$ and $b=\mu+\varepsilon_{1}$, where $\left|\varepsilon_{1}\right| \ll 1$. Because of available medical care in NZ very few individuals actually die from rheumatic fever meaning $f$ is very close to 1 . Most of the deaths are due to Chronic Rheumatic Heart Disease, which can develop with reccuring episodes of acute rheumatic fever.

Let $(1-f) \kappa=\varepsilon_{2},\left|\varepsilon_{2}\right| \ll 1$. This means 
$\frac{d N}{d t} \approx\left(\mu+\varepsilon_{1}\right) N-(1-f) \kappa A-\mu N=\varepsilon_{1} N+\varepsilon_{2} A$.

$\varepsilon_{1} N>\varepsilon_{2} A$, So $\frac{d N}{d t}<2 \varepsilon_{1} N$.

If we let $\varepsilon_{1} t=\tau, \frac{d N}{d \tau}<2 N$.

The fact that $N$ is only noticeably changing on the timescale $\tau=\varepsilon t$, means that with respect to $t$ we can consider $N$ to be constant and treat it as such in our analysis.

Treating $N=S+I+A$ as a constant and $b \approx \mu$ we find two equilibrium points.

$$
(S, I)=(N, 0) \text { and }\left(\frac{\gamma+\omega+\mu}{\beta}, \frac{(\mu+\kappa f)(\beta N-\gamma+\mu+\omega)}{\beta(\omega+\kappa f+\mu)}\right)
$$

The Jacobian matrix for this system 5.1 is

$$
\left[\begin{array}{cc}
-\beta I-\kappa f-\mu & -\beta S+\gamma-\kappa f \\
\beta I & \beta S-\omega-\gamma-\mu
\end{array}\right]
$$

At the disease free equilibrium $(N, 0)$, this becomes

$$
\left[\begin{array}{cc}
-\kappa f-\mu & -\beta N+\gamma-\kappa f \\
0 & \beta N-\omega-\gamma-\mu
\end{array}\right]
$$

This has two eigenvalues $-\kappa f-\mu$ and $\beta N-\gamma-\omega-\mu$. This means the disease free equilibrium for this system is stable as long as $\beta N<\gamma+\omega+\mu$.

At the endemic equilibrium the Jacobian matrix becomes

$$
\left[\begin{array}{cc}
\frac{(\mu+\kappa f)(\gamma+\omega+\mu-\beta N)}{\omega+\kappa f+\mu}-\kappa f-\mu & -\omega-\mu-\kappa f \\
\frac{(\mu+\kappa f)(\beta N-\gamma-\mu-\omega)}{\omega+\kappa f+\mu} & 0
\end{array}\right]
$$

We will find the stability of this point using the trace and determinant. The trace is

$$
\frac{(\mu+\kappa f)(\gamma+\omega+\mu-\beta N)}{\omega+\kappa f+\mu}-\kappa f-\mu=\frac{(\kappa f+\mu)(\gamma-\kappa f-\beta N)}{\omega+\kappa f+\mu}
$$


This is negative as long as $\beta N>\gamma-\kappa f$.

The determinant of 5.4 is

$$
(\mu+\kappa f)(\beta N-\gamma-\mu-\omega)
$$

This is positive when $\beta N>\gamma+\mu+\omega$. So the endemic equilibrium can only be stable when the disease free equilibrium is unstable. Again we see an example of transcritical bifurcation. The endemic equilibrium is stable as long as $\beta N>$ $\gamma+\omega+\mu$.

\subsection{Stochastic Model with Vital Dynamics}

Using our rheumatic fever model with vital dynamics from 5.1, with $b=\mu N$, we can derive a set of transition probabilities for the CTMC SIAS model with vital dynamics. Using $S=N-A-I$ the probabilities of each single change of state are as follows

$$
\begin{aligned}
& p_{(i+j, a+k),(i, a)}(\Delta t)=
\end{aligned}
$$

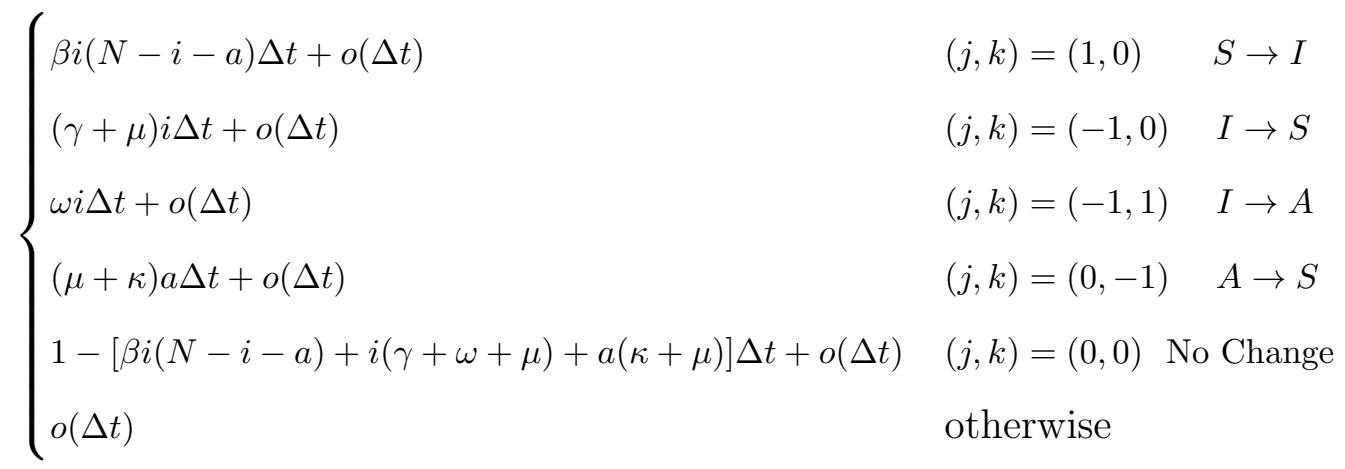

$\mu i$ and $\mu a$ represent the birth of a susceptible being balanced by the death of an infected or an individual with acute rheumatic fever, respectively.

The probability that $I=i$ and $A=a$ at time $t+\Delta t$ in terms of the transition 


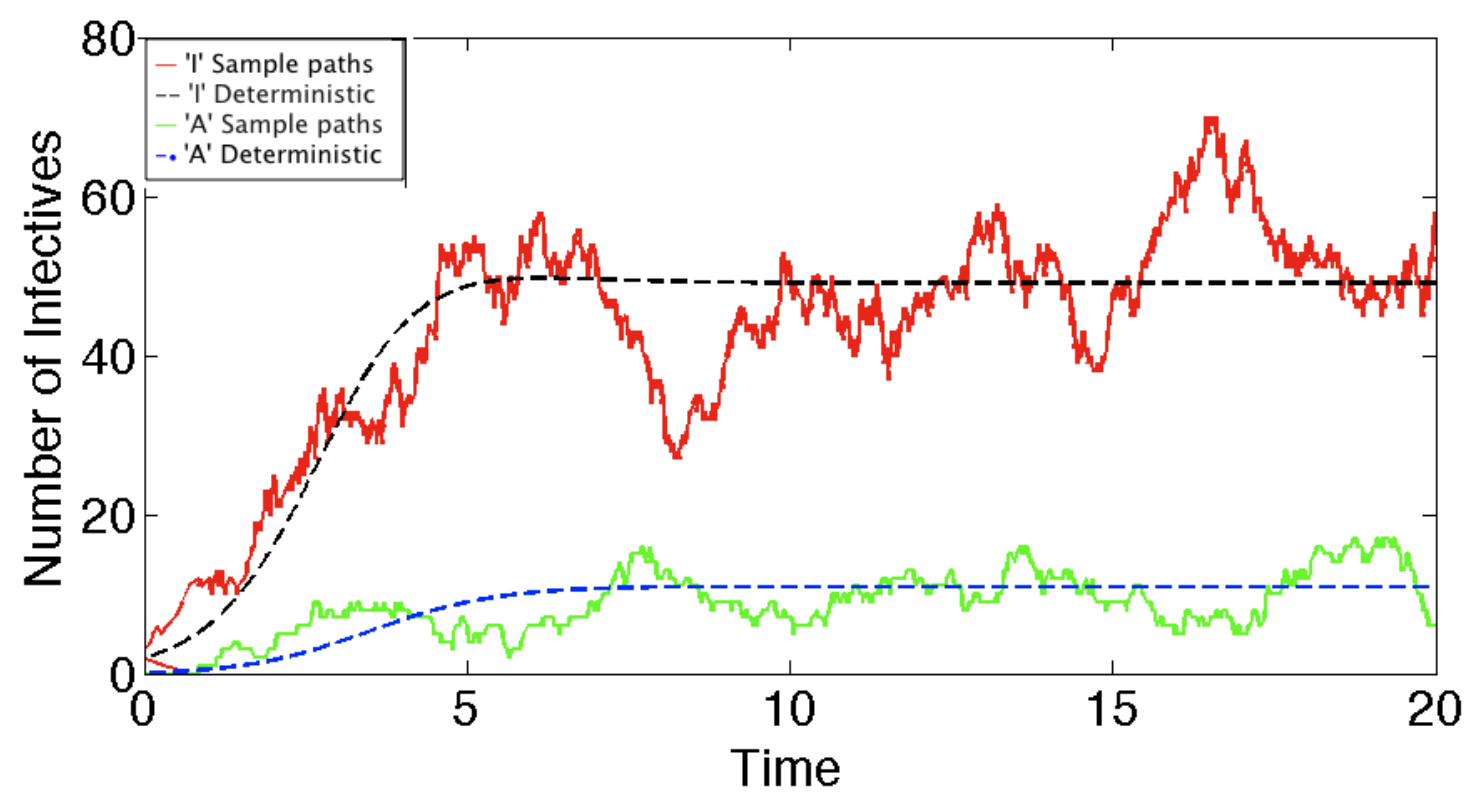

Figure 5.2: Two sample paths of the CTMC SI AS model given by the transition probabilities in 5.5. The solid lines are the CTMC sample paths. The dashed lines show the deterministic model. The lower set of lines is the number of individuals with acute rheumatic fever (A) and the higher are the number with Group A streptococcus (I). $N=100, \beta=0.02, \mu=0.1, \gamma=0.5 . \omega=0.2, \kappa=0.8$

probabilities is given by

$$
\begin{aligned}
p_{(i, a)}(t+\Delta t) & =p_{(i-1, a)}(t) \beta(i-1)(N-i+1-a) \Delta t \\
& +p_{(i+1, a)}(t)(\gamma+\mu)(i+t) \Delta t+p_{(i+1, a-1)}(t) \omega(i+1) \Delta t \\
& +p_{(i, a+1)}(t)(\kappa+\mu)(a+1) \Delta t \\
& +p_{(i, a)}(t)(1-[\beta i(N-i-a)+i(\gamma+\omega+\mu)+a(\kappa+\mu)] \Delta t)
\end{aligned}
$$

A plot of two sample paths, using these transition probabilities is shown in Figure 5.2. Matlab was used to create this plot

Basically we use a random number between 0 and 1 to pick which type of change occurs in each time step. The size of each time step is randomly chosen on an exponential distribution as was discussed in section 4.4.2. The code for this is in the appendix. 


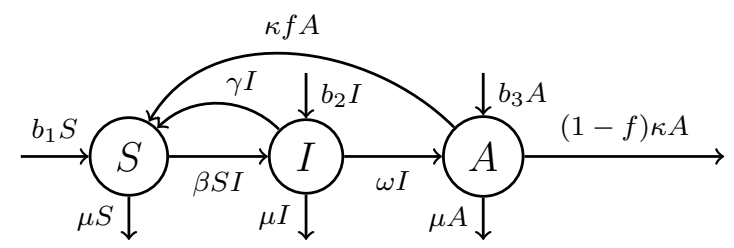

Figure 5.3: Flow chart for the SIAS epidemic model with migration and vital dynamics, given in 5.7 .

\subsection{Migration}

In New Zealand, migration can be an important factor. Immigration must be represented slightly differently from births, as individuals could possibly be infected with Group A streptococcus or have acute rheumatic fever when they migrate. This means we need three separate 'birth terms'. Let $b_{1} S+b_{2} I+b_{3} A=b N$ be the total rate of inflow into the population due to births and immigration. $b_{1}$ is a combination of susceptible immigrants and births of children, $b_{2}$ represents immigration of infected individuals and $b_{3}$ represents the immigration of individuals with acute rheumatic fever. Now $\mu$ represents deaths and emigration. This gives us a modified system of equations

$$
\begin{aligned}
& \frac{d S}{d t}=b_{1} S-\beta S I+\gamma I+\kappa f A-\mu S \\
& \frac{d I}{d t}=b_{2} I+\beta S I-\gamma I-\omega I-\mu I \\
& \frac{d A}{d t}=b_{3} A+\omega I-\kappa A-\mu A
\end{aligned}
$$

and consequentially,

$$
\frac{d N}{d t}=b N-(1-f) \kappa A-\mu N
$$

This modification to the equations alters the flow chart by having entry into each compartment via immigration. This is shown in Figure 5.3 .

Using the argument in section 5.1.1 above, we will treat the population size $N$, as a constant. This means we can again reduce our system so we are just dealing 
with $I$ and $S$.

The Jacobian matrix we then get is

$$
\left[\begin{array}{cc}
b_{1}-\beta I-\kappa f-\mu & -\beta S+\gamma-\kappa f \\
\beta I & b_{2}+\beta S-\omega-\gamma-\mu
\end{array}\right]
$$

Setting the equations in 5.7 equal to 0 we get $I=0$ or $\beta S=\omega+\gamma+\mu-b_{2}$.

$I=0$ gives us the disease free equilibrium

$$
S=\frac{\kappa f N}{\kappa f+\mu-b_{1}}
$$

Substituting this into the Jacobian matrix (5.9) we get

$$
\left[\begin{array}{cc}
b_{1}-\kappa f-\mu & -\frac{\beta \kappa f N}{\kappa f+\mu-b_{1}}+\gamma-\kappa f \\
0 & b_{2}+\frac{\beta \kappa f N}{\kappa F+\mu-b_{1}}-\omega-\gamma-\mu
\end{array}\right]
$$

which has eigenvalues $b_{1}-\kappa f-\mu$ and $b_{2}+\frac{\beta \kappa f N}{\kappa f+\mu-b_{1}}-\omega-\gamma-\mu$. These are both negative if

$$
b_{1}<\kappa f+\mu \text { and } \beta N<\frac{\left(\kappa f+\mu-b_{1}\right)\left(\omega+\gamma+\mu-b_{2}\right)}{\kappa f}
$$

$\beta N>0$ always, so for the second condition to be true, the righthand side of the inequality must also be positive. The first condition means $\kappa f+\mu-b_{1}>0$ so we need $\omega+\gamma+\mu>b_{2}$. Under these conditions the disease free equilibrium is stable.

The endemic equilibrium exists at $\beta S=\omega+\gamma+\mu-b_{2}$ and

$$
\beta I=\frac{\beta \kappa f N-\left(\kappa f+\mu-b_{1}\right)\left(\omega+\gamma+\mu-b_{2}\right)}{\omega+\mu+\kappa f-b_{2}}
$$


The Jacobian matrix at the endemic equilibrium then becomes

$$
\left[\begin{array}{cc}
\frac{(\kappa f-\gamma)\left(b_{1}-\kappa f-\mu\right)-\beta \kappa f N}{\omega+\mu+\kappa f-b_{2}} & -\omega-\mu+b_{2}-\kappa f \\
\frac{\beta \kappa f N-\left(\kappa f+\mu-b_{1}\right)\left(\omega+\gamma+\mu-b_{2}\right)}{\omega+\mu+\kappa f-b_{2}} & 0
\end{array}\right]
$$

The determinant of the Jacobian matrix 5.11 is

$$
\beta \kappa f N-\left(\kappa f+\mu-b_{1}\right)\left(\omega+\gamma+\mu-b_{2}\right)
$$

This is positive if

$$
\beta \kappa f N>\left(\kappa f+\mu-b_{1}\right)\left(\omega+\gamma+\mu-b_{2}\right)
$$

The trace of 5.11 is

$$
\frac{(\kappa f-\gamma)\left(b_{1}-\kappa f-\mu\right)-\beta \kappa f N}{\omega+\mu+\kappa f-b_{2}}
$$

which is negative if

$$
\beta \kappa f N>(\kappa f-\gamma)\left(b_{1}-\kappa f-\mu\right)
$$

Because the endemic equilibrium exists at $\beta S=\omega+\gamma+\mu-b_{2}$, we need $\beta N \geq$ $\omega+\gamma+\mu-b_{2}$ for this equilibrium point to be valid (All compartments contain non-negative numbers of individuals). So for the endemic equilibrium to be both valid and stable we need

$$
\beta N>\frac{\left(\kappa f+\mu-b_{1}\right)\left(\omega+\gamma+\mu-b_{2}\right)}{\kappa f} \geq \omega+\gamma+\mu-b_{2}
$$

Which implies

$$
\begin{aligned}
\frac{\kappa f+\mu-b_{1}}{\kappa f} & \geq 1 \\
\kappa f+\mu-b_{1} & \geq \kappa f \\
\mu-b_{1} & \geq 0 \\
\mu & \geq b_{1}
\end{aligned}
$$


This makes sense under the assumption that $N$ is approximately constant and $\mu N \approx b N=b_{1} S+b_{2} I+b_{3} A$. We are also assuming $\kappa f+\omega+2 \gamma+\mu>b_{2}$ though this seems to be a reasonable assumption given all our parameters are positive and $b_{2}$, which is the rate of infected people entering the population, is likely to be quite small.

\subsubsection{Independence of Immigration}

In the above system 5.7, rates of migration and births into and from each compartment are represented as proportional to the number of individuals currently in that compartment. This makes sense for deaths and emigration, as the number of people available to leave a compartment would be dependent on how many are in that compartment. For births and immigration however, it makes more sense for the number for people entering to be dependent on the whole population size, or even independent of any other value. Infected individuals and individuals with acute rheumatic fever will not immigrate based on how many other individuals are infected or have acute rheumatic fever, it is much more likely to depend on the total population size. Although this still may not be true in New Zealand where immigration is regulated, it may also be more dependent on the size of the global population. Taking this into account we can modify the equations to get this system.

$$
\begin{aligned}
& \frac{d S}{d t}=b_{1} N-\beta S I+\gamma I+\kappa f A-\mu S \\
& \frac{d I}{d t}=b_{2} N+\beta S I-\gamma I-\omega I-\mu I \\
& \frac{d A}{d t}=b_{3} N+\omega I-\kappa A-\mu A
\end{aligned}
$$

The rates of immigration into each compartment are now all proportional to $N$ instead of each individual compartment size. the differential equation for $N$ remains the same as in 5.7. Figure 5.4 shows the modification to the flow chart.

Using the same argument as above in section 5.1, we will treat the population size, $N$, as a constant. This means we can again reduce our system so we are just 


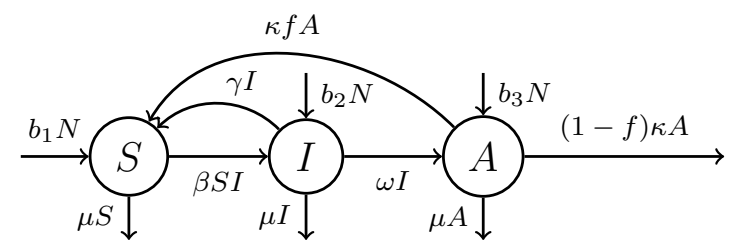

Figure 5.4: Flow chart for the $S I A S$ epidemic model with immigration proportional to total population size as given in 5.12 .

dealing with $I$ and $S$. The Jacobian matrix we then get is

$$
\left[\begin{array}{cc}
-\beta I-\kappa f-\mu & -\beta S+\gamma-\kappa f \\
\beta I & \beta S-\omega-\gamma-\mu
\end{array}\right]
$$

Setting the equations in the system equal to 0 we get

$$
\begin{aligned}
& S=\frac{(\gamma+\mu+\omega) I-b_{2} N}{\beta I} \\
& I=\frac{\beta N+\gamma+\omega+\mu \pm \sqrt{(\beta N+\gamma+\omega+\mu)^{2}-4 \beta b_{2} N\left(\frac{\omega}{\kappa+\mu}+1\right)}}{2 \beta\left(\frac{\omega}{\kappa+\mu}+1\right)}
\end{aligned}
$$

These are both the endemic equilibria. Because there is always the possibility of infected persons entering the population via immigration there is no disease free equilibrium.

\subsection{Exposed Periods and Treatment Models}

In this section we will be deviating briefly from acute rheumatic fever modelling and focusing more generally on SIR type models. Not everything mentioned in this section relates explicitly to acute rheumatic fever, but there are important concepts here that we need to consider. We will discuss the use of these concepts in the later development of our SIAS model in Chapter 6 . 


\subsubsection{Exposed Periods}

Many infectious diseases have a latent period, where an individual has been exposed to the disease and is infected, but is not yet infectious. The length of this exposed period has little effect on the predictions of an epidemic model [7]. To incorporate an exposed period into an SIR type model, we add the compartment $E$. With a mean exposed period of $1 / v$ this gives us the $S E I R$ model below:

$$
\begin{aligned}
\frac{d S}{d t} & =-\beta S I \\
\frac{d E}{d t} & =\beta S I-v E \\
\frac{d I}{d t} & =v E-\gamma I \\
\frac{d N}{d t} & =-(1-f) \gamma I
\end{aligned}
$$

We have used the equation for $N$ instead of $R$ as it shows our inclusion of the possibility for death from the disease better. The equation for $R$ is $R^{\prime}=\gamma f I$ as not all infectious individuals recover.

In some diseases, this exposed period is really an asymptomatic stage of infectiousness, where there is some infectiousness but it is reduced by some factor $\varepsilon_{E}$ until the individual moves into the fully infectious compartment. In this case the equations for $S$ and $E$ become

$$
\begin{aligned}
& S^{\prime}=-\beta S\left(I+\varepsilon_{E} E\right) \\
& E^{\prime}=\beta S\left(I+\varepsilon_{E} E\right)-v E
\end{aligned}
$$

The equations for $I$ and $N$ remain the same as in 5.14 [7]. For this model $\mathcal{R}_{0}$ is the sum of the secondary infections caused by an individual in a fully susceptible population, during their exposed period and during their infectious period.

$$
\mathcal{R}_{0}=\frac{\beta N}{\gamma}+\varepsilon_{E} \frac{\beta N}{\kappa}
$$

Though we will not be using exposed periods in the acute rheumatic fever model we 
develop in this thesis, the concept of more that one compartment being infectious is important [7]. We will use multiple infectious compartments later on along with the idea that $\mathcal{R}_{0}$ is the sum of all the secondary infections caused by each compartment.

\subsubsection{Vaccination models}

For many diseases, there are vaccinations to protect against infection. This is one form of treatment, and a simple way of accounting for it is to reduce the population size by the number of vaccinated individuals. In reality however vaccinations may only reduce the rate of infection. Along with other treatments however, they may reduce the infectiousness of a vaccinated individual if they do become infected [7]. Modelling this requires an extra compartment similar to the exposed compartment in section 5.4.1 above. In the case of vaccination however, vaccinated individuals follow a path parallel to other individuals. Not everyone goes though the compartment of reduced infectiousness.

The main issue with modelling this separation is identifying who has been vaccinated prior to becoming infected. What this may require is multiple compartments for susceptible individuals. This will keep vaccinated individuals separate from the rest of the population in the model so we can deal with their interactions in the population separately.

If we use the subscript $V$ to identify vaccinated individuals, the flow chart for an $S I R$ model with vaccination might look like the diagram shown in Figure 5.5 .

Once an individual is recovered from the infection, with immunity, their previous vaccination status is no longer important to the model.

We have not included vital dynamics in this model. The main point of its introduction is to show the use of multiple susceptible compartments and parallel infection and recovery paths. The differential equations that match the diagram in Figure 5.5 are

$$
\frac{d S}{d t}=-\beta S\left(I+\varepsilon I_{V}\right)-\lambda S \quad \frac{d S_{V}}{d t}=\lambda S-\kappa S_{V}\left(I+\varepsilon I_{V}\right)
$$




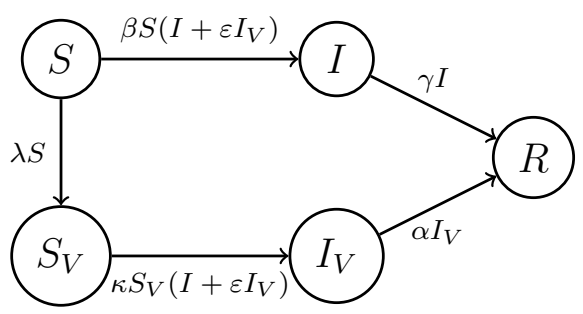

Figure 5.5: A flow chart for the SIR model with vaccination. $\kappa$ represents the reduced susceptibility of vaccinated individuals. $\varepsilon$ is the factor by which their infectiousness is reduced. $\lambda$ is the rate of vaccination.

$$
\begin{aligned}
\frac{d I}{d t} & =\beta S\left(I+\varepsilon I_{V}\right)-\gamma I & \frac{d I_{V}}{d t}=\kappa S_{V}\left(I+\varepsilon I_{V}\right)-\alpha I_{V} \\
\frac{d R}{d t} & =\gamma I+\alpha I_{V} &
\end{aligned}
$$

Though it may be unrealistic, this model assumes that the vaccination lasts lifetime. this is purely to provide a simple introduction to this type of model, a more complicated model could be constructed to account for vaccination wearing off over time, but is beyond the scope of this thesis. We are also assuming mixing in the population is independent of vaccination status. This means any susceptible individual, vaccinated or not, can be infected by any infectious individual. $\mathcal{R}_{0}$ is the sum of the number of new infections due to each infectious compartment. It is the rate of transfer of individuals into that compartment multiplied by the rate at which they leave. If we let $I+I_{V}=I_{t}$ be the total number of infectious individuals, the part of $\mathcal{R}_{0}$ due to $I$ is as follows

$$
\frac{\beta S I_{t}}{\beta S I_{t}+\kappa S_{V} I_{t}} \times \frac{\beta S I+\kappa S_{V} I}{\gamma I}=\frac{\beta S}{\gamma}
$$

$\beta S I_{t}$ is the portion of individuals being infected who move into compartment $I$. $\beta S I+\kappa S_{V} I$ is Th rate of infection due to individuals in compartment $I$, and $\gamma I \mathrm{i}$ the rate that they leave $I$.

Doing the same thing for compartment $I_{V}$ we get

$$
\frac{\kappa S_{V} I_{t}}{\beta S I_{t}+\kappa S_{V} I_{t}} \times \frac{\beta S \varepsilon I_{V}+\kappa S_{V} \varepsilon I_{V}}{\alpha I_{V}}=\varepsilon \frac{\kappa S_{V}}{\alpha}
$$


This gives us

$$
\mathcal{R}_{0}=\frac{\beta S}{\gamma}+\varepsilon \frac{\kappa S_{V}}{\alpha}
$$

The $\varepsilon$ is the factor by which infectiousness of vaccinated is reduced.

There is currently no vaccination against Group A streptococcus, available for widespread use [12]. Because of this we will not use a vaccination compartment in our further development of the model for acute rheumatic fever. Both Group A streptococcus and acute rheumatic fever however are diseases that have shown increased prevalence among certain age groups [4] and in New Zealand, different ethnicities show variation [18][11]. This is an area where we may need to use multiple susceptible compartments to account for the different rates of infection for different demographics.

\subsubsection{Treatment for infection}

In the situation where there is a treatment for the disease after an individual has been infected, we can use an SITR model, where $T$ is the compartment containing those undergoing treatment. Suppose some fraction $\alpha$ of infected individuals are selected for treatment per unit of time, the treatment reduces infectivity by a fraction $\delta$ and the rate of removal of treated individuals is $\eta$. [7]. The model we then produce is

$$
\begin{aligned}
& \frac{d S}{d t}=-\beta S(I+\delta T) \\
& \frac{d I}{d T}=\beta S(I+\delta T)-(\gamma+\alpha) I \\
& \frac{d T}{d t}=\alpha I-\eta T \\
& \frac{d R}{d t}=\gamma I+\eta T
\end{aligned}
$$

The flow chart for this model is shown in Figure 5.6

The mean amount of time spent in the infective compartment is $1 /(\gamma+\alpha)$ so an individual in this compartment would cause $\beta N /(\gamma+\alpha)$ secondary infections, in a fully susceptible population. The fraction $\alpha /(\gamma+\alpha)$ of infected individuals are 


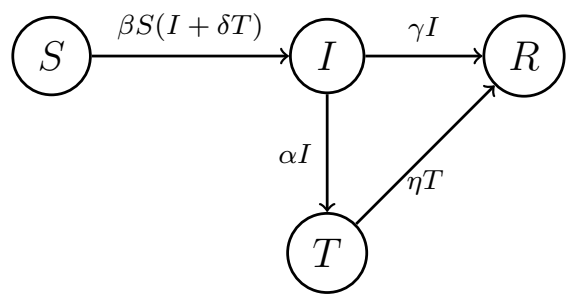

Figure 5.6: A flow chart for the $S I R$ model with treatment as seen in 5.18, $\delta$ is the factor by which infectiousness is reduced for individuals in treatment. $\alpha$ is the rate of treatment. $\gamma$ and $\eta$ represent the different rates of recovery.

treated. While in the treatment compartment an individual will cause $\delta \beta N$ new infections per unit of time. Individuals remain in the treatment class for a mean time of $1 / \eta$ so the number of secondary infections caused by treated individuals, in a fully suscpetible population, is $\alpha /(\gamma+\alpha) \times \delta \beta N / \eta[7]$. Overall $\mathcal{R}_{0}$ is

$$
\mathcal{R}_{0}=\frac{\beta N}{\gamma+\alpha}+\frac{\alpha \delta \beta N}{\eta(\gamma+\alpha)}
$$

\subsection{Acute Rheumatic Fever History}

An individual's history in relation to acute rheumatic fever will affect their likelihood of developing the disease following their next Group A streptococcus infection. An individual who has had acute rheumatic fever before is more at risk than someone who has not [1]. This is something we should account for in our model. Similarly to how the population was split in section 5.4 .2 based on vaccination status, we can split the population based on individual history in relation to acute rheumatic fever. We assume that an individual's history of acute rheumatic fever only affects their susceptibility to acute rheumatic fever and not their susceptibility or infectiousness in relation to Group A streptococcus. Figure 5.7 shows what the flow of individuals would look like in a model accounting for acute rheumatic fever history.

The subscript $A$ represents those individuals who have had Acute Rheumatic fever in the past. These individuals have a greater chance of developing acute rheumatic 


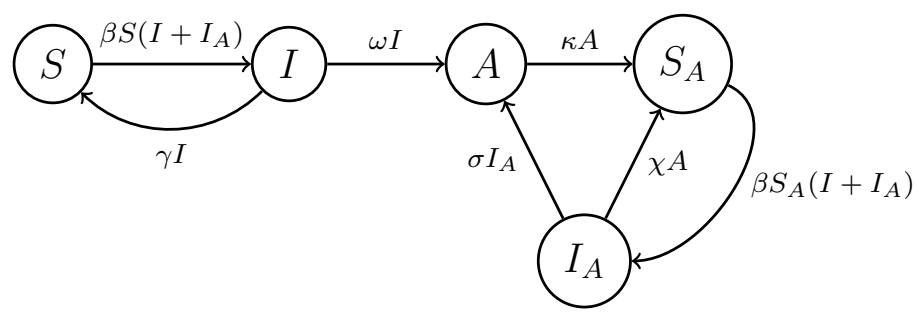

Figure 5.7: Flow chart for an acute rheumatic fever model with compartments for individuals with a history of Rheumatic fever.

fever, so $\sigma>\omega$. It is also logical that they would have a reduced chance of recovering from a Group A streptococcus infection without developing acute rheumatic fever, therefore $\chi<\gamma$. The differential equations for the model shown in Figure 5.7 are given in 5.20 below

$$
\begin{array}{rlrl}
\frac{d S}{d t}=-\beta S\left(I+I_{A}\right)+\gamma I & \frac{d S_{A}}{d t} & =\kappa A-\beta S_{A}\left(I+I_{A}\right)+\chi I_{A} \\
\frac{d I}{d t}=\beta S\left(I+I_{A}\right)-\gamma I-\omega I & \frac{d I_{A}}{d t} & =\beta S_{A}\left(I+I_{A}\right)-\chi I_{A}-\sigma I_{A} \\
\frac{d A}{d t} & =\omega I+\sigma I_{A}-\kappa A
\end{array}
$$

One big issue with this model is that all individuals flow towards eventually having acute rheumatic fever. $S$ gets replenished by those who recover from Group A streptococcus without developing acute rheumatic fever. Some individuals however, are always lost to the right hand side of the diagram in Figure 5.7. Eventually there will be no one left without a history of acute rheumatic fever. We can see this in the equilibrium points as well.

The differential equations in 5.20 give us two equilibrium points. The disease free equilibrium occurs when

$$
I=A=I_{A}=0 \Longrightarrow S+S_{A}=N
$$

It is realistic for individuals to settle into the two susceptible compartments once the disease dies out and can theoretically occur at any value of $S$ or $S_{A}$.

The other endemic equilibrium however, can only be reached once we run out of 


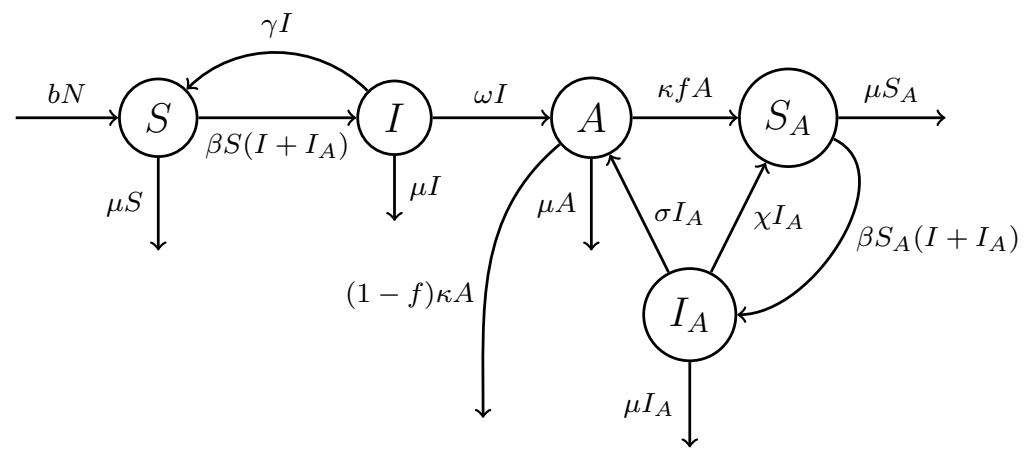

Figure 5.8: Flow chart for an acute rheumatic fever model with compartments for individuals with a history of acute rheumatic fever and vital dynamics.

individuals who have never had acute rheumatic fever. This happens when

$$
S=I=0, \quad S_{A}=\frac{\chi+\sigma}{\beta}, \quad I_{A}=\frac{\kappa(\chi+\sigma)}{\beta(\kappa+\sigma)} \text { and } A=\frac{\sigma(\chi+\sigma)}{\beta(\kappa+\sigma)}
$$

This is an unrealistic equilibrium, especially in a population where not everyone is actually susceptible to developing acute rheumatic fever.

A model that is unrealistic is not much use in the real world. We need to do something about the fact that we are running out of susceptible individuals with no history of acute rheumatic fever. One way to balance this loss is by introducing new individuals in the form of newborns. This means we need to include vital dynamics in the model.

\subsubsection{History with Vital Dynamics}

By including vital dynamics we can replenish the susceptible compartment containing those with no history of acute rheumatic fever. Figure 5.8 shows the flow of individuals once we add in the vital dynamics.

The introduction of vital dynamics also allows us to include the possibility that individuals might die from acute rheumatic fever. The system of differential equa- 
tions when we include vital dynamics and disease deaths is as follows:

$$
\begin{aligned}
\frac{d S}{d t} & =b N-\beta S\left(I+I_{A}\right)+\gamma I-\mu S \\
\frac{d I}{d t} & =\beta S\left(I+I_{A}\right)-\gamma I-\omega I-\mu I \\
\frac{d A}{d t} & =\omega I+\sigma I_{A}-\kappa A-\mu A \\
\frac{d S_{A}}{d t} & =\kappa f A-\beta S_{A}\left(I+I_{A}\right)+\chi I_{A}-\mu S_{A} \\
\frac{d I_{A}}{d t} & =\beta S_{A}\left(I+I_{A}\right)-\chi I_{A}-\sigma I_{A}-\mu I_{A}
\end{aligned}
$$

where $b$ is the birth rate. $\mu$ is the death rate and $(1-f)$ is the proportion of individuals that die from acute rheumatic fever. If we let $b \approx \mu$ as per the argument in section 5.1.1 we can find the equilibrium points. The equations in 5.21 give us three equilibrium points. The disease free equilibrium occurs when

$$
S=N, \text { and } I=A=S_{A}=I_{A}=0
$$

This describes the situation where Group A streptococcus has died out in the population before anyone has developed acute rheumatic fever and survived it. Or everyone with a history of acute rheumatic fever has died out.

If $f \neq 1$ The endemic equilibrium we find is

$$
\begin{array}{ll}
S=-\frac{\sigma(\gamma+\omega+\mu)}{\beta(\omega-\sigma)} & S_{A}=\frac{\omega(\chi+\sigma+\mu)}{\beta(\omega-\sigma)} \\
I=\frac{\sigma \mu(\sigma+\chi+\mu)}{\beta(\omega \mu-\sigma(\sigma+\omega+\mu))} & I_{A}=-\frac{\omega \mu(\sigma+\chi+\mu)}{\beta(\omega \mu-\sigma(\sigma+\omega+\mu))} \\
A=0 &
\end{array}
$$

Even though $f \neq 1$, none of these equations depend on $f$, because $\kappa f$ is the coefficient of $A$ and $A=0$ at this equilibrium.

This equilibrium however is not valid. If all of the compartments and parameters are non-negative, $S$ and $S_{A}$ and $I$ and $I_{A}$ cannot validly exist at the same time. 
For $S$ to be valid we need

$$
\begin{aligned}
S=-\frac{\sigma(\gamma+\omega+\mu)}{\beta(\omega-\sigma)} & \geq 0, \quad \omega \neq \sigma \\
\beta(\omega-\sigma) & <0 \\
\omega & <\sigma
\end{aligned}
$$

However for $S_{A}$ to be valid we require that $\omega>\sigma$, the opposite. The only way this point could be valid is if $\sigma=0$. The equilibrium point would then become

$$
S=I=A=0, \text { and } S_{A}=I_{A}=\frac{\chi+\mu}{\beta}
$$

Which like the equilibrium point in section 5.5 above is unrealistic and acutally impossible if $\mu>0$. So this model has no realistic endemic equilibrium unless, under the assumption $\mu \approx b$, the assumption that $f \approx 1$ is also made .

The running reproduction number for this model is

$$
\mathcal{R}^{*} \frac{\beta S}{\gamma+\omega+\mu}+\frac{\beta S_{A}}{\chi+\sigma+\mu}
$$

For the numerical solution 5.9 If we assumed the outbreak started in a fully susceptible population with no history of acute rheumatic fever, then basic reproduction number would be

$$
\mathcal{R}_{0}=\frac{\beta N}{\gamma+\omega+\mu}
$$

Figure 5.9 shows a plot a numerical solution produced by this model. In this particular plot $\mathcal{R}_{0}=3.85$. The number of infected individuals shoots up steeply then begins to settle down to what looks like an endemic equilibrium.

By assuming $f=1$ we get three equilibrium points, one of which is the disease free equilibrium given above. The other two equilibrium points points, are endemic equilibria. They are solutions of cubic which we will not show here. The numbers 


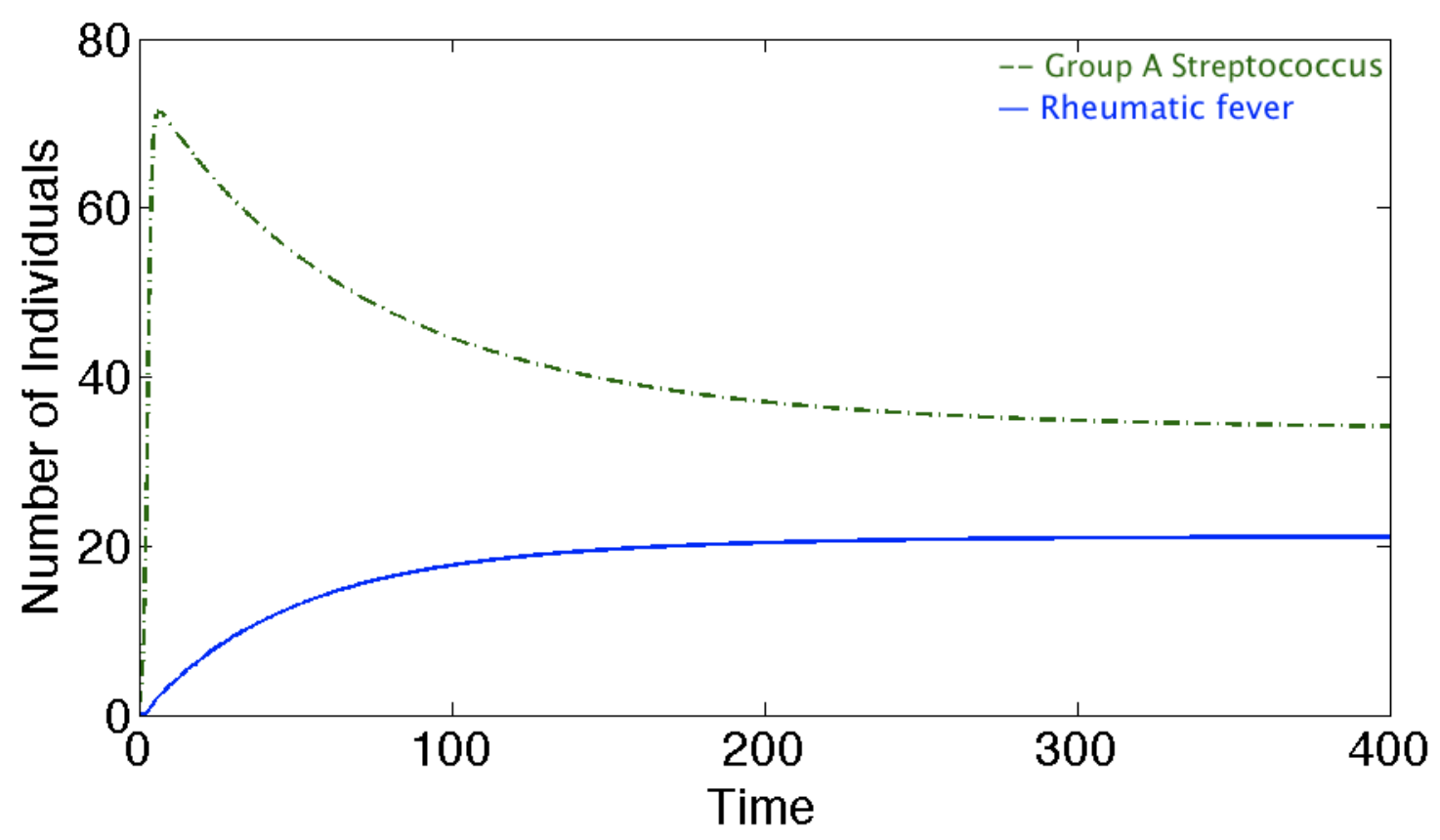

Figure 5.9: A plot of numerical solutions for acute rheumatic fever history model given in 5.21. The dashed, green line represents total Group A streptococcus numbers, both compartment $I$ and $I_{A}$ added together. The solid line plots the acute rheumatic fever $(A)$ numbers. $N=100, \beta=0.02, \mu=0.01, \gamma=0.5 . \omega=$ $0.02, \kappa=0.8, \chi=0.4, \sigma=0.5$. 
in each compartment in terms of $S$ however are;

$$
\begin{aligned}
I & =\frac{\mu(N-S)}{\omega+\mu} \\
I_{A} & =\frac{\mu(S-N)(\beta S-\gamma-\omega-\mu)}{\beta S(\omega+\mu)} \\
A & =\frac{\mu(N-S)(\omega \beta S-\sigma(\beta S-\gamma-\omega-\mu))}{\beta S(\omega+\mu)(\kappa+\mu)} \\
S_{A} & =\frac{\beta S(\chi+\sigma+\omega)(\omega+\gamma+\mu-\beta S)}{\omega+\gamma+\mu}
\end{aligned}
$$

If we use $\mathcal{R}^{*}=1$ at an endemic equilibrium we can find the values of $S$ at the equilibrium points from equation 5.22 .

$$
\begin{aligned}
& \mathcal{R}^{*}=\frac{\beta S}{\gamma+\omega+\mu}+\frac{\beta S(\chi+\sigma+\omega)(\gamma+\omega+\mu-\beta S)}{(\gamma+\omega+\mu)(\chi+\sigma+\mu)}=1 \\
& \frac{\beta S+\beta S(\gamma+\omega+\mu)-(\beta S)^{2}}{\gamma+\omega+\mu}=1 \\
& \beta S(\beta S-1)-(\gamma+\omega+\mu)(\beta S-1)=0 \\
&(\beta S-(\gamma+\omega+\mu))(\beta S-1)=0 \\
& \Rightarrow \beta S=\gamma+\omega+\mu \quad \text { or } \quad \beta S=1 .
\end{aligned}
$$




\section{Chapter 6}

\section{A New Zealand Specific Model}

In New Zealand, acute rheumatic fever is a notifiable disease. This means that if an individual is diagnosed with acute rheumatic fever, the medical practitioner involved must report it [18, 22]. Because of this requirement data for rates of acute rheumatic fever in New Zealand are fairly good.

To construct a model that follows the behaviour of acute rheumatic fever in New Zealand accurately, we need to take into account aspects of the disease's behaviour that are specific to New Zealand.

There is a strong ethnic association with acute rheumatic fever in New Zealand. Maori and Pacific Island peoples have an increased risk of developing the disease following a Group A streptococcus infection. Rates also seem to vary geographically within the country [18]. It is not clear if this geographical variation is due to the distribution of ethnicities in the country, or if the increased incidence among particular ethnicities is due to higher rates in different geographical locations. It is possible it could be a mix of both variables. Even if we are not sure of the cause, these factors should be taken into account in our model.

Age is also a factor in an individual's risk levels. While it is not specific to New Zealand it is an important factor that we need to account for in the construction of our model. 


\subsection{Public Health records and treatment}

Because acute rheumatic fever is a notifiable disease in New Zealand, and because of good public health records, anyone who has had acute rheumatic fever once will be treated differently in the future in relation to Group A streptococcus and acute rheumatic fever symptoms. This means that as people recover from an episode of acute rheumatic fever, we cannot just put them back into the susceptible population with everyone else. We need to add extra compartments to the model for individuals with a history of acute rheumatic fever, as we did in section 5.5 .

The effective treatment of Group A streptococcus will prevent the development of acute rheumatic fever [24]. When we include treatment classes in our model, we allow the assumption that individuals who receive treatment for Group A streptococcus will not go on to develop acute rheumatic fever. We can also assume that individuals with a known history of acute rheumatic fever will always seek treatment for a Group A streptococcus infection.

If we start with a simple model without any vital dynamics we produce the following system of equations.

$$
\begin{aligned}
\frac{d S}{d t} & =-\beta S\left(I+I_{A}\right)+\gamma I+\rho T \\
\frac{d I}{d t} & =\beta S\left(I+I_{A}\right)-\gamma I-\phi I-\omega I \\
\frac{d T}{d t} & =\phi I-\rho T \\
\frac{d A}{d t} & =\omega I-\eta A \\
\frac{S_{A}}{d t} & =\eta A+\lambda T_{A}-\beta S_{A}\left(I+I_{A}\right) \\
\frac{d I_{A}}{d t} & =\beta S_{A}\left(I+I_{A}\right)-\sigma I_{A} \\
\frac{d T_{A}}{d t} & =\sigma I_{A}-\lambda T_{A}
\end{aligned}
$$

$T_{A}, S_{A}$, and $I_{A}$ represent individuals who have been through the acute rheumatic fever compartment and are now in treatment, susceptible or infected with Group A streptococcus respectively. Figure 6.1 shows the flow of individuals for this 


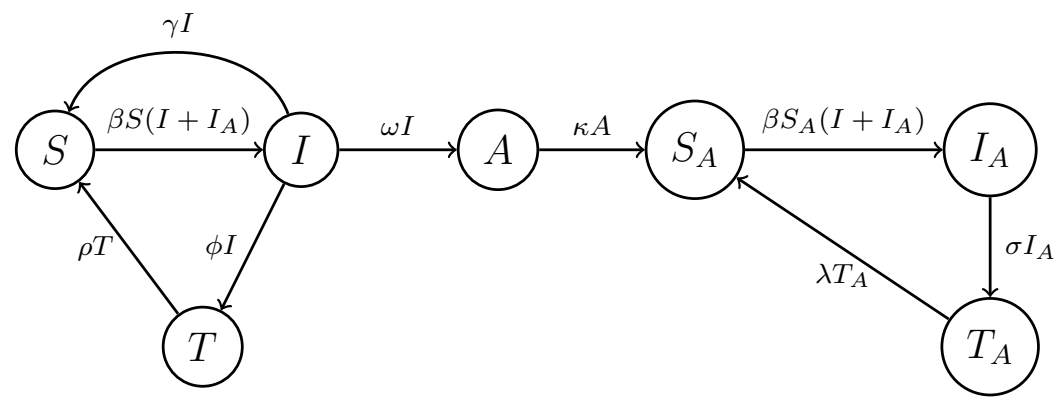

Figure 6.1: Flow of individuals in an acute rheumatic fever treatment model with compartments for individuals with a history of acute rheumatic fever, as modelled by the equations in 6.1 .

system.

Because of the way we have set up the rate of transfer between compartments, once an individual has gone through the acute rheumatic fever compartment they cannot go back to being a 'normal' susceptible. This causes a bit of a problem though because eventually everyone in the population is going to go through the acute rheumatic fever compartment as we have set up no alternative route. This is similar to what we saw in section 5.5 for the system 5.20 .

We get two possible equilibrium points for this model. The disease free equilibrium is at $\left(S, I, T, A, S_{A}, I_{A}, T_{A}\right)=(S, 0,0,0, N-S, 0,0)$ where the disease dies out, this can happen at any point in time during the epidemic. This equilibrium is stable as long as $\beta N<\sigma$.

This model also has an endemic equilibrium at

$$
\left(S, I, I, A, S_{A}, I_{A}, T_{A}\right)=\left(0,0,0,0, \frac{\sigma}{\beta}, \frac{\sigma(\beta N-\sigma)}{\beta \lambda(\lambda+1)}, \frac{\beta N-\sigma}{\beta(\lambda+1)}\right)
$$

The Jacobian for this equilibrium has a determinant of 0 and the trace is

$$
-\frac{2 \lambda(\beta N-\sigma)}{\sigma-\lambda}-\eta-\phi-\rho-\omega-\lambda-\gamma
$$


This means this equilibrium point is stable as long as

$$
\frac{2 \lambda(\beta N-\sigma)}{\sigma-\lambda}>0
$$

So either

$$
\beta N>\sigma>\lambda \text { or } \lambda>\sigma>\beta N
$$

This equilibrium seems rather unrealistic, especially when we know that not everyone will develop acute rheumatic fever after contracting Group A streptococcus, even if they have multiple Group A streptococcus infections [11]. To solve this problem we need to allow for people to recover from Group A streptococcus and never develop acute rheumatic fever, introducing vital dynamics could help here.

\subsubsection{Vital dynamics model}

Including vital dynamics in the model allows us to bring new susceptible individuals into the population via births. At the same time individuals with a history of acute rheumatic fever may leave the population via deaths. If we assume 'birth rate' $=$ 'death rate' $=\mu$, the new system of equations we get is

$$
\begin{aligned}
\frac{d S}{d t} & =\mu N-\beta S\left(I+I_{A}\right)+\gamma I+\rho T-\mu S \\
\frac{d I}{d t} & =\beta S\left(I+I_{A}\right)-\gamma I-\phi I-\omega I-\mu I \\
\frac{d T}{d t} & =\phi I-\rho T-\mu T \\
\frac{d A}{d t} & =\omega I-\eta A-\mu A \\
\frac{d S_{A}}{d t} & =\eta A+\lambda T_{A}-\beta S_{A}\left(I+I_{A}\right)-\mu S_{A} \\
\frac{d I_{A}}{d t} & =\beta S_{A}\left(I+I_{A}\right)-\sigma I_{A}-\mu I_{A} \\
\frac{d T_{A}}{d t} & =\sigma I_{A}-\lambda T_{A}-\mu T_{A}
\end{aligned}
$$

One of these equations is redundant, if we let $T_{A}=N-\left(S+I+T+A+S_{A}+I_{A}\right)$ 


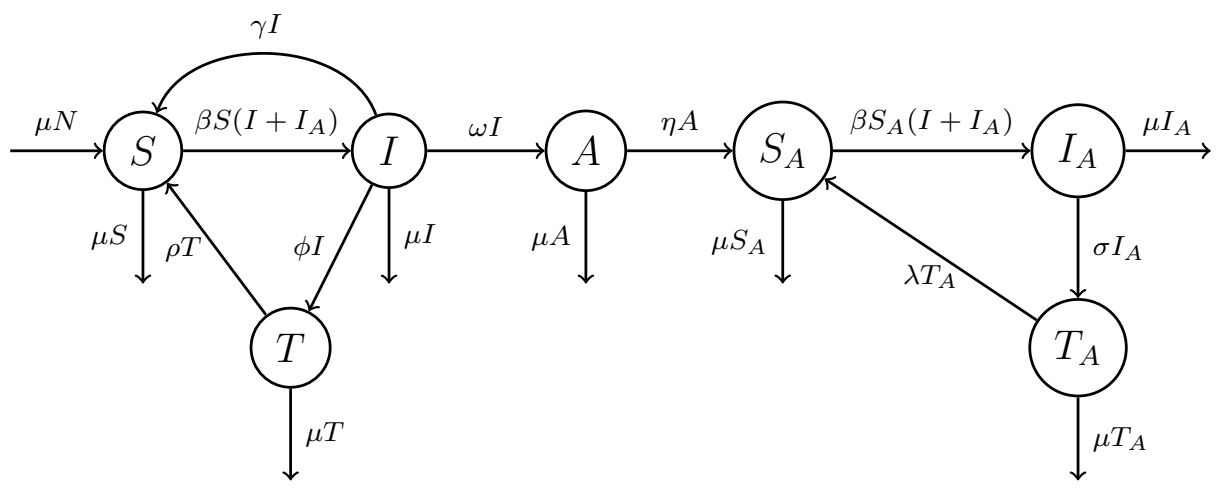

Figure 6.2: Flow chart for an acute rheumatic fever treatment model with vital dynamics and compartments for individuals with a history of acute rheumatic fever.

we get the jacobian matrix

$$
\left[\begin{array}{cccccc}
-\beta\left(I+I_{A}\right)-\mu & -\beta S+\gamma & \rho & 0 & 0 & -\beta S \\
\beta\left(I+I_{A}\right) & \beta S-\mu-\phi-\omega-\gamma & 0 & 0 & 0 & \beta S \\
0 & \phi & -\mu-\rho & 0 & 0 & 0 \\
0 & \omega & 0 & -\mu-\eta & 0 & 0 \\
-\lambda & -\beta S_{A}-\lambda & -\lambda & \eta-\lambda & -\beta\left(I+I_{A}\right)-\lambda-\mu & -\beta S_{A}-\lambda \\
0 & \beta S_{A} & 0 & 0 & \beta\left(I+I_{A}\right) & \beta S_{A}-\mu-\sigma
\end{array}\right]
$$

A diagram showing the flow of individuals for this model is shown in Figure 6.2. In this case, as most acute rheumatic fever deaths are due to recurrent attacks that lead to rheumatic heart disease [12, we have simplified the model by assuming there are no deaths due to the disease. Because of the way we have designed the model, individuals with a history of acute rheumatic fever always receive treatment for a Group A streptococcus infection. They therefore never develop acute rheumatic fever again. We have essentially eliminated the possibility of recurrent attacks in individuals with a known history of acute rheumatic fever. We are also assuming that births equal deaths so our population size $N$ is constant.

Using Sage [30] we were able to find two equilibrium points for this model. The disease free equilibrium at;

$$
S=N, \text { and } I=T=A=S_{A}=I_{A}=T_{A}=0
$$


This is similar to what we saw in section 5.5.1. The disease free equilibrium point represents a population where Group A streptococcus has died out before anyone has developed acute rheumatic fever. Because we have included vital dynamics in this model, it is also possible there were individuals who had acute rheumatic fever, but all these compartments have died out.

The Jacobian matrix in the case of the disease free equilibrium becomes

$$
\left[\begin{array}{ccccccc}
0 & -\beta N+b+\rho+\gamma & \mu & \mu & \mu & -\beta N+\mu & \mu \\
0 & \beta N-\mu-\phi-\omega-\gamma & 0 & 0 & 0 & \beta N & 0 \\
0 & \phi & -\mu-\rho & 0 & 0 & 0 & 0 \\
0 & \omega & 0 & -b-\eta & 0 & 0 & 0 \\
0 & 0 & 0 & \eta & -\mu & 0 & \lambda \\
0 & 0 & 0 & 0 & 0 & -\mu-\sigma & 0 \\
0 & 0 & 0 & 0 & 0 & \sigma & -\mu-\lambda
\end{array}\right]
$$

This equilibrium point is stable as long as $\beta N<\mu+\phi+\omega+\gamma$.

Analytically there is another equilibrium point at

$$
\begin{array}{ll}
S=I=A=0 & S_{A}=\frac{\sigma+\mu}{\beta} \\
I_{A}=-\frac{(\lambda+\mu)(\sigma+\mu)}{\beta(\lambda+\sigma+\mu)} & T_{A}=-\frac{\sigma(\sigma+\mu)}{\beta(\lambda+\sigma+\mu)}
\end{array}
$$

This point immediately appears unrealistic and it is. Much like the endemic equilibrium point in section 5.5, it is unrealistic for the whole population to develop rheumatic fever.

This point is also not valid. The compartment sizes cancel each other out by all adding to 0 instead of $N$, and both $I_{A}$ and $T_{A}$ have negative values. For a biologically realistic model all compartments must be non-negative in size. Therefore this model also has no valid endemic equilibrium.

The basic reproduction number for this model, $\mathcal{R}_{0}$ is

$$
\mathcal{R}_{0}=\frac{\beta N}{\mu+\phi+\gamma+\omega}
$$




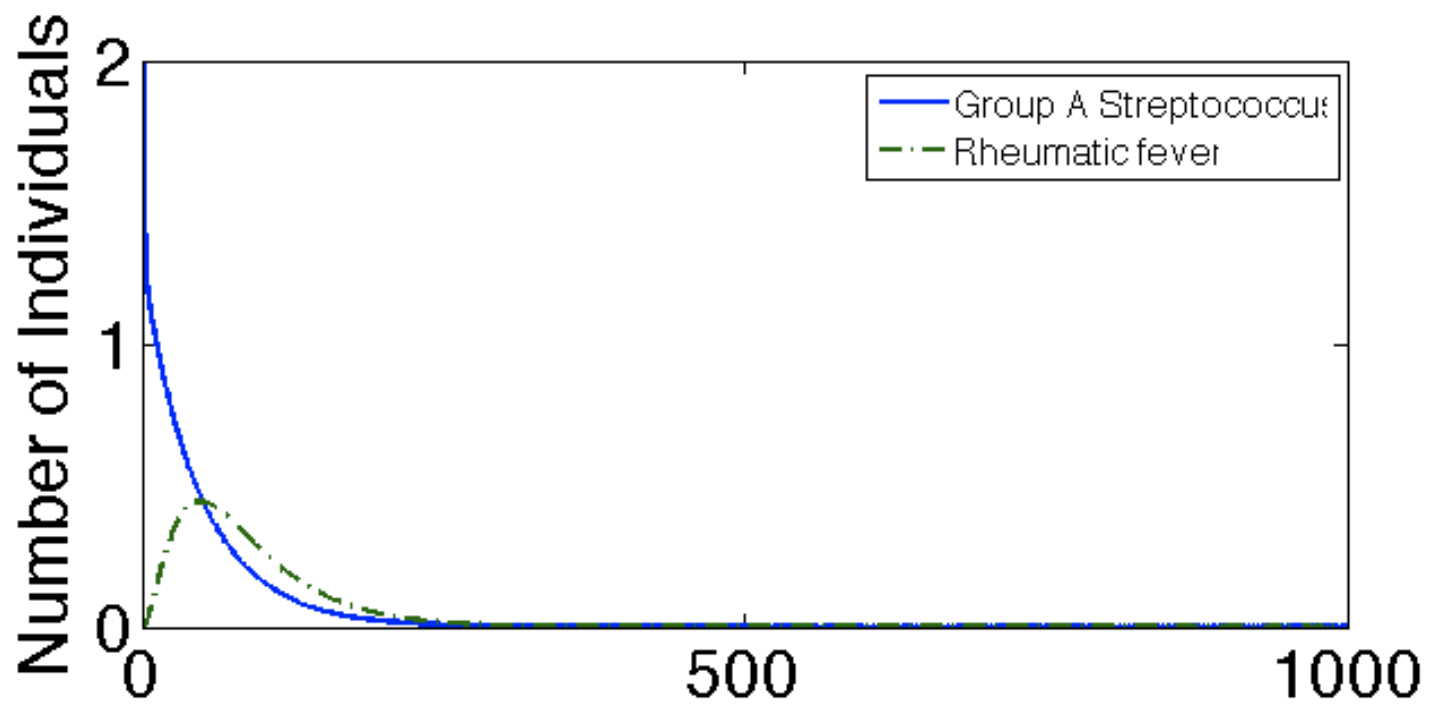

\section{Time}

Figure 6.3: Numerical solution for the model given in 6.2. Group A streptococcus $\left(I+I_{A}\right)$ is represented by the solid line and acute rheumatic fever $(A)$ numbers are given by the dashed line. $\mathcal{R}_{0}<1$. The initial number of infections was 2 . $\mathrm{N}=400$, $\beta=0.00055, \mu=0.01, \phi=0.1, \omega=0.03, \gamma=0.1, \sigma=0.8$

as time goes on and individuals develop acute rheumatic fever, moving into the right hand side of the model, our effective reproduction number becomes

$$
\mathcal{R}^{*}=\frac{\beta S}{\mu+\phi+\gamma+\omega}+\frac{\beta S_{A}}{\mu+\sigma}
$$

as infectious individuals with a history of acute rheumatic fever also effect the rate of infection.

When $\mathcal{R}_{0}<1, \beta N<\mu+\phi+\omega+\gamma$. The disease dies out as expected and we reach the disease free equilibrium, $I=I_{A}=0$. Figure 6.3 shows how the number of infectious individuals quickly dies out in this scenario and the number of individuals with acute rheumatic fever follows closely.

There is an initial bump in the number of individuals with acute rheumatic fever as a proportion of those infected with Group A streptococcus before it dies out, 


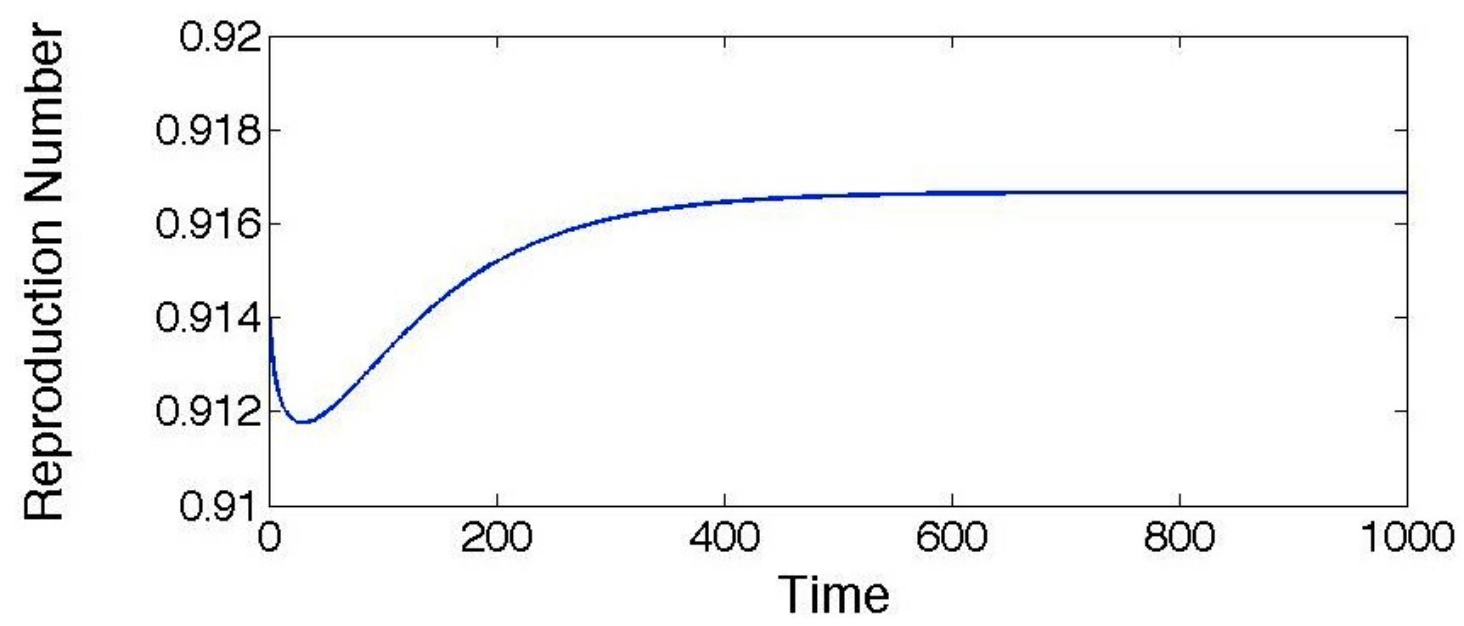

Figure 6.4: Plot of the reproduction number with $\mathcal{R}_{0}<1$, using numerical solutions to the model given in 6.2. The same parameters as used in Figure 6.3 apply.

still develop acute rheumatic fever.

With the parameters, used for the plot in Figure 6.3, $\mathcal{R}_{0}=0.914<1$. As some individuals are infected and develop acute rheumatic fever initially the effective reproduction number drops with the falling number of susceptible. Once individuals start to recover faster than they are being infected, the reproduction number starts to increase. The disease continues to die out however because there are no infectious individuals left to infect anyone and $\mathcal{R}^{*}<1$ still. The effective reproduction number settles at $\mathcal{R}_{0}$. This behaviour is shown in Figure 6.4.

We were unable to find a realistic endemic equilibrium easily earlier. So what does happen when $\beta N>b+\omega+\gamma+\phi$ ? Figure 6.5 shows a plot for $\beta N>\mu+\phi+\omega+\gamma$ and $\mathcal{R}_{0}>1$.

The number of infected individuals shoots up steeply near the start but then drops off almost as steeply to settle into an equilibrium. The number of individuals with acute rheumatic fever does the same thing but not quite so steeply.

The effective reproduction number drops off sharply near the beginning, and actually drops below one twice before settling there in an equilibrium. This is shown in Figure 6.6. From these Figures 6.3, 6.4, 6.5 and 6.6, we can see the disease free equilibrium is reached when $\mathcal{R}^{*}=\mathcal{R}_{0}$ and the endemic equilibrium when $\mathcal{R}^{*}=1$. 


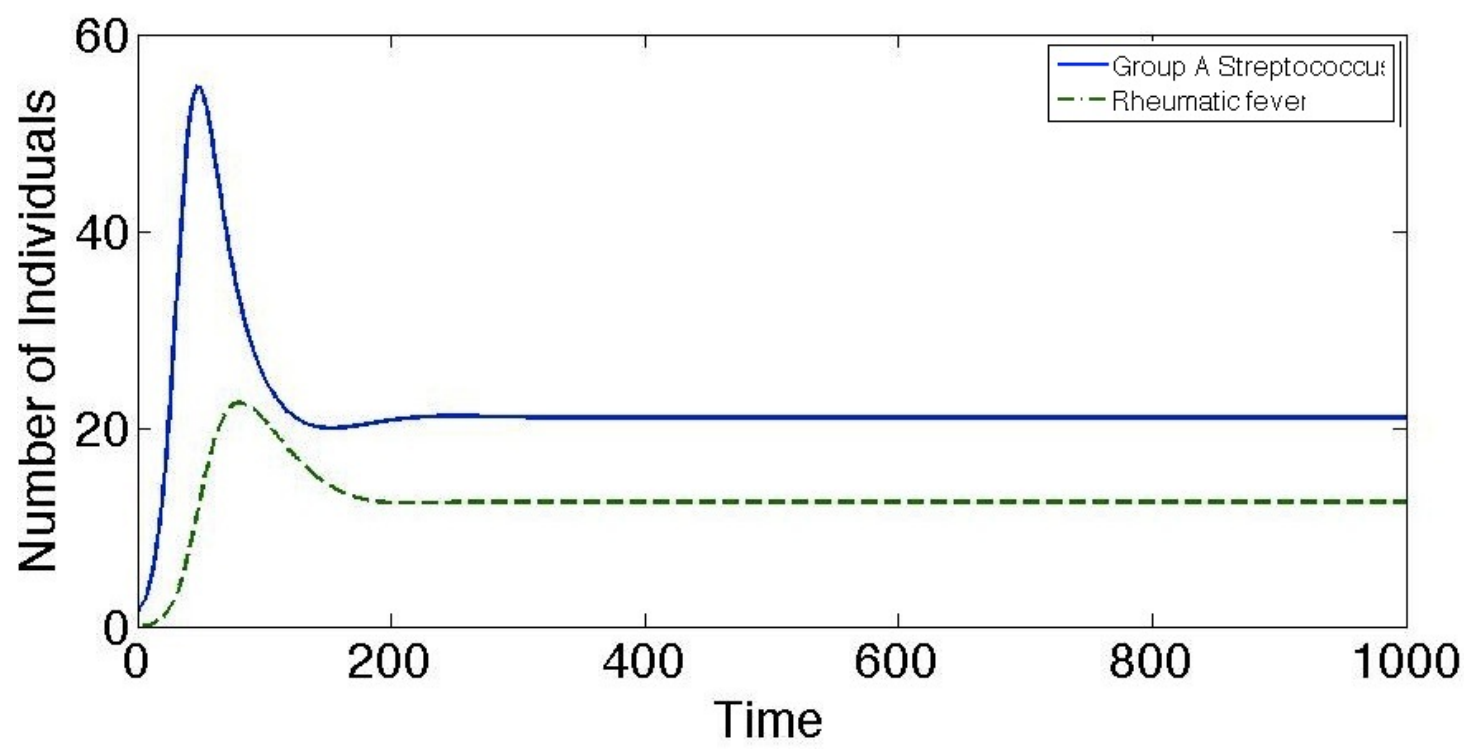

Figure 6.5: Group A streptococcus and acute rheumatic fever numbers, given by numerical solutions to the model seen in $6.2, \mathcal{R}_{0}>1$. The dashed line represents rheumatic fever and the solid line gives Group A streptococcus numbers. The initial number of infectious was 2 . $\mathrm{N}=400, \beta=0.0009, \mu=0.01, \phi=0.1, \omega=$ $0.03, \gamma=0.1, \sigma=0.8$

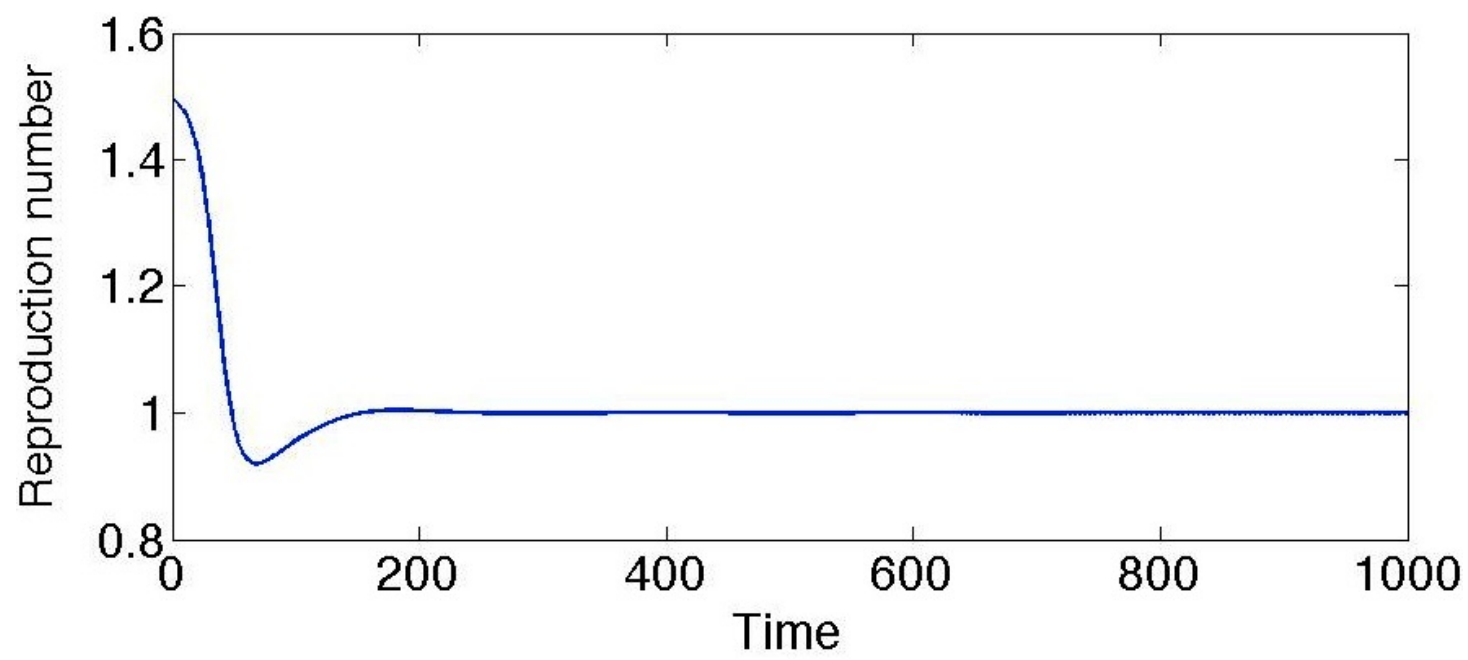

Figure 6.6: Plot of the running reproduction number for $\mathcal{R}_{0}>1$, using numerical soulutions to the model given in 6.2. The same parameters as used in Figure 6.5 apply. 


\begin{tabular}{|c|c|c|c|c|}
\hline $\mathbf{i}$ & $\mathbf{j}$ & Probability & Change in i & Change in j \\
\hline $\mathrm{S}$ & $\mathrm{I}$ & $\beta S\left(I+I_{A}\right) \Delta t+o(\Delta t)$ & $s \longrightarrow s-1$ & $i \longrightarrow i+1$ \\
\hline $\mathrm{I}$ & $\mathrm{S}$ & $(\gamma+\mu) I \Delta t+o(\Delta t)$ & $i \longrightarrow i-1$ & $s \longrightarrow s+1$ \\
\hline $\mathrm{I}$ & $\mathrm{T}$ & $\phi I \Delta t+o(\Delta t)$ & $i \longrightarrow i-1$ & $\tau \longrightarrow \tau+1$ \\
\hline $\mathrm{T}$ & $\mathrm{S}$ & $(\rho+\mu) T \Delta t+o(\Delta t)$ & $\tau \longrightarrow \tau-1$ & $\mathrm{~s} \longrightarrow \mathrm{s}+1$ \\
\hline $\mathrm{I}$ & $\mathrm{A}$ & $\omega I \Delta t+o(\Delta t)$ & $i \longrightarrow i-1$ & $a \longrightarrow a+1$ \\
\hline $\mathrm{A}$ & $\mathrm{S}$ & $\mu A \Delta t+o(\Delta t)$ & $a \longrightarrow a-1$ & $s \longrightarrow s+1$ \\
\hline $\mathrm{A}$ & $\mathrm{S}_{A}$ & $\eta A \Delta t+o(\Delta t)$ & $a \longrightarrow a-1$ & $s_{A} \longrightarrow s_{A}+1$ \\
\hline $\mathrm{T}_{A}$ & $\mathrm{~S}$ & $\mu T_{A} \Delta t+o(\Delta t)$ & $\tau_{A} \longrightarrow \tau_{A}-1$ & $s \longrightarrow s+1$ \\
\hline $\mathrm{T}_{A}$ & $S_{A}$ & $\lambda T_{A} \Delta t+o(\Delta t)$ & $\tau_{A} \longrightarrow \tau_{A}-1$ & $s_{A} \longrightarrow s_{A}+1$ \\
\hline $\mathrm{S}_{A}$ & $\mathrm{~S}$ & $\mu S_{A} \Delta t+o(\Delta t)$ & $s_{A} \longrightarrow s_{A}-1$ & $s \longrightarrow s+1$ \\
\hline $\mathrm{S}_{A}$ & $\mathrm{I}_{A}$ & $\beta S_{A}\left(I+I_{A}\right) \Delta t+o(\Delta t)$ & $s_{A} \longrightarrow s_{A}-1$ & $i_{A} \longrightarrow i_{A}+1$ \\
\hline $\mathrm{I}_{A}$ & $\mathrm{~S}$ & $\mu I_{A} \Delta t+o(\Delta t)$ & $i_{A} \longrightarrow i_{A}-1$ & $s \longrightarrow s+1$ \\
\hline $\mathrm{I}_{A}$ & $\mathrm{~T}_{A}$ & $\sigma I_{A} \Delta t+o(\Delta t)$ & $i_{A} \longrightarrow i_{A}-1$ & $\tau_{A} \longrightarrow \tau_{A}+1$ \\
\hline
\end{tabular}

Table 6.1: Transition probabilities for transition of individuals from compartment $\mathrm{i}$ to $\mathrm{j}$. The probability that nothing changes is one minus, the sum of all the above probabilities.

So we can confirm the presence of the endemic equilibrium by plotting a numerical solution, and estimate when it will happen using the running reproduction number $\mathcal{R}^{*}$. The question stability is still unanswered.

\subsubsection{Stochastic Model}

We can easily derive a CTMC Model incorporating individual acute rheumatic fever history, treatment and vital dynamics.

Using a stochastic model allows us to incorporate chance into the model. We can essentially account for unknown factors and influences on the patterns of disease spread. The stochastic variation allows for a more accurate prediction of what might actually happen without the need to complicate the actual model further.

The table of transition probabilities is shown in Table 6.1 Using these transition probabilities and the same parameters as we did for the deterministic model we can plot sample paths. Figure 6.7 shows two sample paths for the stochastic model, plotted along with the deterministic model. 


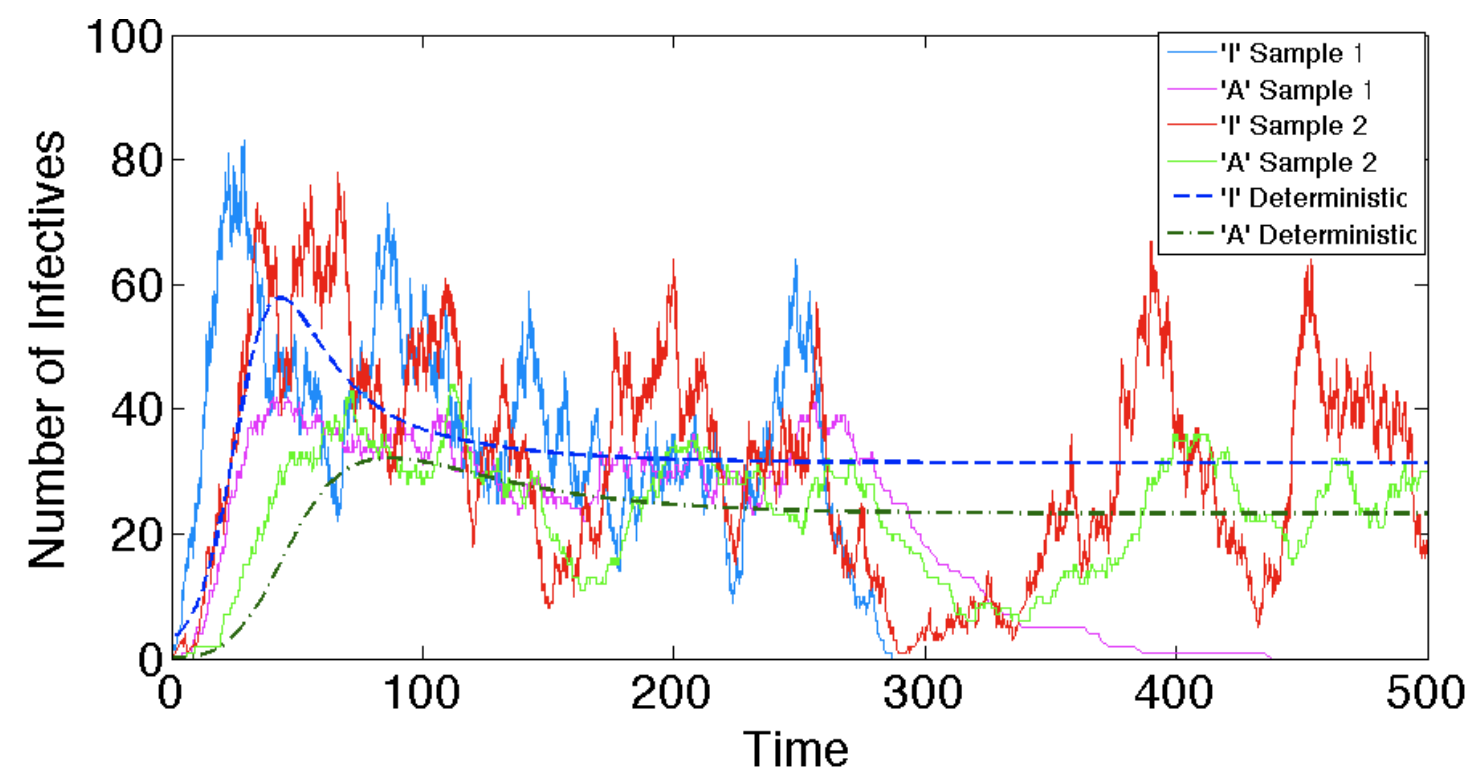

Figure 6.7: CTMC plot using the transition probabilities given in table 6.1. The parameter values are the same as those used to produce the plot in figure 6.5. The dashed lines show the deterministic model. ' $I$ ' is the number of individuals infected with Group A streptococcus, ' $A$ ' is the number of indiviuals suffering from acute rheumatic fever. 
The stochastic model tends to oscillate a lot. In one of the simulations, Group A streptococcus died out just before 200 days, even though the deterministic plot shows the disease settling to an equilibrium. This is an example of a the disease can die out at anytime in the stochastic model no matter what the deterministic model predicts should happen. It also suggests that the endemic equilibrium may not be stable.

\subsection{Including Demographics}

The risks of both contracting Group A streptococcus and developing acute rheumatic fever change with age and vary due to ethnicity and geographic location. Because of this variation we need to take these demographics into account when constructing our model. Individuals of different ages and ethnicities need to be separated in the model so we can account for their differing rates of developing acute rheumatic fever. A simple variation of the $S I A S$ model that accounts for ethnicity is shown below:

$$
\begin{aligned}
\frac{d S_{M}}{d t} & =-\beta S_{M}\left(I_{M}+I_{E}\right)+\gamma I_{M}+\kappa A_{M} \\
\frac{d I_{M}}{d t} & =\beta S_{M}\left(I_{M}+I_{E}\right)-\gamma I_{M}-\omega I_{M} \\
\frac{d A_{M}}{d t} & =\omega I_{M}-\kappa A_{M} \\
\frac{d S_{E}}{d t} & =-\beta S_{E}\left(I_{M}+I_{E}\right)+\gamma I_{E}+\kappa A_{E} \\
\frac{d I_{E}}{d t} & =\beta S_{E}\left(I_{M}+I_{E}\right)-\gamma I_{E}-\alpha I_{E} \\
\frac{d A_{E}}{d t} & =\alpha I_{E}-\kappa A_{E}
\end{aligned}
$$

The M and E subscripts denote Maori \& Pacific Island peoples and other ethnicities respectively. $\omega$ and $\alpha$ represent the different rates of developing acute rheumatic fever for Maori and Pacific peoples and the other ethnicities. There is no movement of individuals between ethnic subgroups. Assuming homogeneous mixing however, infectious individuals of any ethnicity can infect any susceptible individual at the 

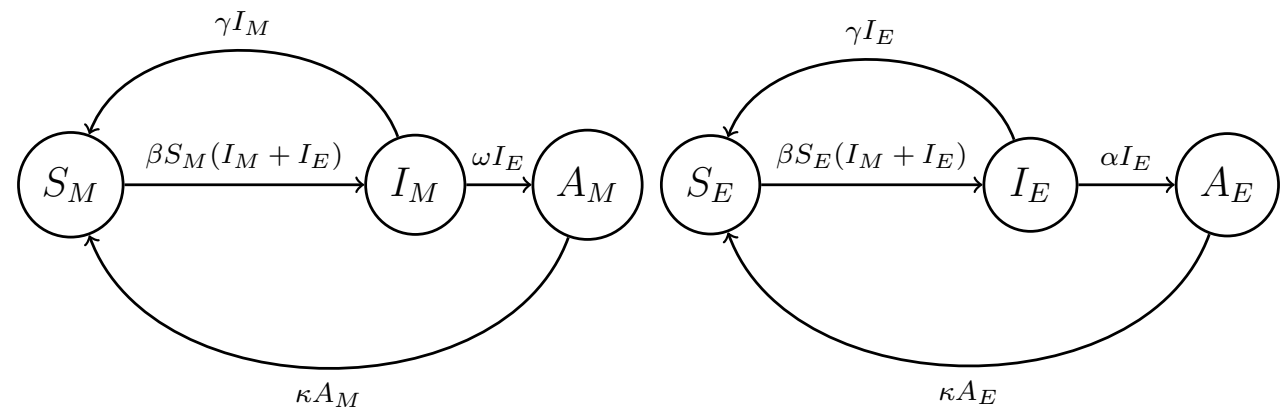

Figure 6.8: Flow chart for an acute rheumatic fever model including ethnicity, given by the equations in 6.6 .

same rate regardless of ethnicity. In reality homogeneous mixing may not be realistic and some type of proportional or preferential mixing maybe more suitable. This adds further complication to the construction of the model however an is outside the scope of this thesis. Figure 6.8 illustrates the flow of individuals for this model.

Analytically there are three equilibrium points for this model. The disease free equilibrium is at

$$
S_{M}+S_{E}=N, \quad I_{M}=A_{M}=I_{E}=A_{E}=0
$$

In this case, because we have not included acute rheumatic fever history in the model, the disease has just died out at some point and everyone has gone back to being susceptible. An individual's ethnicity does not change so we have $S_{M}=N_{M}$ and $S_{E}=N_{E}$ where $N_{M}$ and $N_{E}$ are the constant population sizes for each ethnic group.

Because one of the equations in the system 6.6 is redundant, we can use $A_{E}=$ $N-\left(S_{M}+I_{M}+A_{M}+S_{E}+I_{E}\right)$, the Jacobian matrix for this is given below in 6.7

$$
\left[\begin{array}{ccccc}
-\beta\left(I_{M}+I_{E}\right) & -\beta S_{M}+\gamma & \kappa & 0 & -\beta S_{M} \\
\beta\left(I_{M}+I_{E}\right) & \beta S_{M}-\gamma-\omega & 0 & 0 & \beta S_{M} \\
0 & \omega & -\kappa & 0 & 0 \\
-\kappa & -\beta S_{E}-\kappa & -\kappa & -\beta\left(I_{M}+I_{E}\right)-\kappa & -\beta S_{E}+\gamma-\kappa \\
0 & \beta S_{E} & 0 & \beta\left(I_{M}+I_{E}\right) & \beta S_{E}-\gamma-\alpha
\end{array}\right]
$$


The Jacobian matrix at the disease free equilibrium becomes

$$
\left[\begin{array}{ccccc}
0 & -\beta N_{M}+\gamma & \kappa & 0 & -\beta N_{M} \\
0 & \beta N_{M}-\gamma-\omega & 0 & 0 & \beta N_{M} \\
0 & \omega & -\kappa & 0 & 0 \\
-\kappa & -\beta N_{E}-\kappa & -\kappa & -\kappa & -\beta N_{E}+\gamma-\kappa \\
0 & \beta N_{E} & 0 & 0 & \beta N_{E}-\gamma-\alpha
\end{array}\right]
$$

The trace of this matrix is $\beta N-2 \gamma-\omega-\alpha-2 \kappa$, and the determinant is 0 . So the disease free equilibrium is stable as long as $\beta N<\alpha+2(\gamma+\kappa)+\omega$.

There is an endemic equilibrium at

$$
\begin{gathered}
S_{M}=\frac{(\gamma+\omega) I_{M}}{\beta\left(I_{M}+I_{E}\right)}, I_{M}=\frac{\beta N_{M}(\alpha+\gamma)-(\gamma+\omega)\left(\alpha+\gamma-\beta\left(N_{M}-I_{E}\left(1+\frac{\alpha}{\kappa}\right)\right)\right)}{\beta(\alpha+\gamma)\left(1+\frac{\omega}{\kappa}\right)}, \\
A_{M}=\frac{\omega I_{M}}{\kappa}, S_{E}=\frac{(\alpha+\gamma)\left(1-\frac{I_{M}}{I_{M}+I_{E}}\right)}{\beta}, \\
I_{E}=I_{M}\left(\frac{\gamma+\omega}{\beta\left(N_{M}-I_{M}\left(1+\frac{\omega}{\kappa}\right)\right)}-1\right), A_{E}=\frac{\alpha I_{E}}{\kappa}
\end{gathered}
$$

We also get a third equilibrium point at

$$
\begin{gathered}
S_{M}=\frac{\gamma+\omega}{\beta}, I_{M}=\frac{\kappa(\beta N-\gamma-\omega)}{\beta(\kappa+\omega)}, A_{M}=\frac{\omega(\beta N-\gamma-\omega)}{\beta(\kappa+\omega)}, \\
S_{E}=0, I_{E}=0, A_{E}=0
\end{gathered}
$$

This last equilibrium is basically a degenerate case for only one ethnicity group in the population. by symmetry the case where $N_{M}=0$ is also an equilibrium point. It occurs at the same place as the endemic equilibrium for the original SI AS model 4.1.

\subsubsection{Ethnicity and ARF History}

As we have seen earlier, how likely an individual is to develop acute rheumatic fever also depends on whether they have had acute rheumatic fever before [1]. 


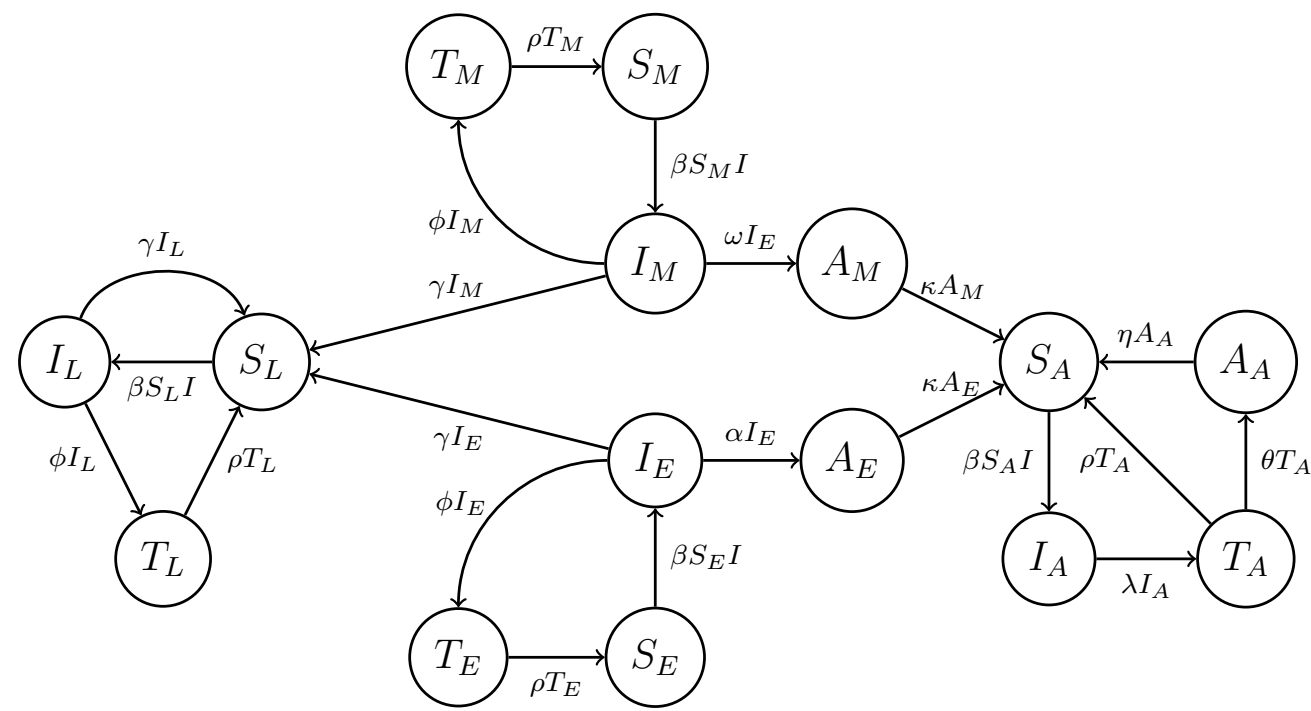

Figure 6.9: Flow chart for an acute rheumatic fever model including ethnicity and rheumatic fever history.

So it makes sense that individuals with a history of acute rheumatic fever be treated differently to those with no history of acute rheumatic fever. Also those who have Group A streptococcus but do not develop acute rheumatic fever even without treatment are less likely to ever develop acute rheumatic fever. It may be useful to treat these individuals separately too. Basically we start with everyone susceptible, in their ethnic groups and then they move into groups based on their history in relation to acute rheumatic fever as Group A streptococcus and acute rheumatic fever spread and develop in the population. Figure 6.9 shows this flow of individuals.

The subscript $L$ is used to denote those who have recovered from a Group A streptococcus infection without treatment and without developing acute rheumatic fever. In this model we then make the assumption that they are not susceptible to acute rheumatic fever so will never develop it.

We have left in the possibility for individuals with a history of acute rheumatic fever to develop it again, even after treatment. This is because of their increased risk of developing the disease and the possibility that treatment may not always be effective in preventing acute rheumatic fever. 
The system of differential equations for the model pictured in figure 6.9 are as follows:

$$
\begin{aligned}
\frac{d S_{M}}{d t} & =-\beta S_{M}\left(I_{M}+I_{E}+I_{A}+I_{L}\right)+\rho T_{M} \\
\frac{d I_{M}}{d t} & =\beta S_{M}\left(I_{M}+I_{E}+I_{A}+I_{L}\right)-I_{M}(\phi+\gamma+\omega) \\
\frac{d T_{M}}{d t} & =\phi I_{M}-\rho T_{M} \\
\frac{d A_{M}}{d t} & =\omega I_{M}-\kappa A_{M} \\
\frac{d S_{E}}{d t} & =-\beta S_{E}\left(I_{M}+I_{E}+I_{A}+I_{L}\right)+\rho T_{E} \\
\frac{d I_{E}}{d t} & =\beta S_{E}\left(I_{M}+I_{E}+I_{A}+I_{L}\right)-I_{E}(\gamma+\alpha+\phi) \\
\frac{d T_{E}}{d t} & =\phi I_{E}-\rho T_{E} \\
\frac{d A_{E}}{d t} & =\alpha I_{E}-\kappa A_{E} \\
\frac{d S_{A}}{d t} & =\kappa\left(A_{M}+A_{E}\right)-\beta S_{A}\left(I_{M}+I_{E}+I_{A}+I_{L}\right)+\rho T_{A}+\eta A_{A} \\
\frac{d I_{A}}{d t} & =\beta S_{A}\left(I_{M}+I_{E}+I_{A}+I_{L}\right)-\lambda I_{A} \\
\frac{d T_{A}}{d t} & =\lambda I_{A}-T_{A}(\rho+\theta) \\
\frac{d A_{A}}{d t} & =\theta T_{A}-\eta A_{A} \\
\frac{d S_{L}}{d t} & =\gamma\left(I_{M}+I_{E}\right)-\beta S_{L}\left(I_{M}+I_{E}+I_{A}+I_{L}\right)+\rho T_{L} \\
\frac{d I_{L}}{d t} & =\beta S_{L}\left(I_{M}+I_{E}+I_{A}+I_{L}\right)-I_{L}(\gamma+\phi) \\
\frac{d T_{L}}{d t} & =\phi I_{L}-\rho T_{L} \\
& =1
\end{aligned}
$$

This system has two equilibria; the disease free equilibrium at

$$
\begin{gathered}
S_{M}+S_{E}+S_{A}+S_{L}=N \\
I_{M}=T_{M}=A_{M}=I_{E}=T_{E}=A_{E}=I_{A}=T_{A}=A_{A}=I_{L}=T_{L}=0
\end{gathered}
$$


and an unrealistic endemic equilibrium that is basically the result of there being no new entry into $S_{M}$ or $S_{E}$. This endemic equilibrium is at

$$
\begin{gathered}
S_{M}=S_{E}=S_{L}=I_{M}=T_{M}=A_{M}=I_{E}=T_{E}=A_{E}=I_{L}=T_{L}=0 \\
S_{A}=\frac{\lambda}{\beta}, I_{A}=\frac{\eta(\rho+\theta)(\beta N-\lambda)}{\beta(\eta(\rho+\theta)+\lambda(\eta+\theta))}, T_{A}=\frac{\lambda I_{A}}{\rho+\theta}, A_{A}=\frac{\lambda \theta I_{A}}{\eta(\rho+\theta)}
\end{gathered}
$$

The trace for the Jacobian matrix at the disease free equilibrium is

$$
\beta S-4 \beta I-\alpha-\eta-3 \gamma-2 \kappa-\lambda-\omega-3 \phi-4 \rho-\theta
$$

Where $S=S_{M}+S_{E}+S_{A}+S_{L}$ and $I=I_{M}+I_{E}+I_{A}+I_{L}$. The determinant is 0 . This means the disease free equilibrium is stable from time zero if

$$
\beta N<\alpha+\eta+3 \gamma+2 \kappa+\lambda+\omega+3 \phi+4 \rho+\theta
$$

For the endemic equilibrium the trace is

$$
-\frac{4(\rho+\theta)(\beta N-\lambda) \eta}{(\rho+\theta) \eta+(\eta+\theta) \lambda}-\alpha-\eta-3 \gamma-2 \kappa-\lambda-\omega-3 \phi-4 \rho-\theta
$$

This makes it stable when

$$
\beta N<\lambda-\frac{(\alpha+\eta+3(\gamma+\phi)+2(\kappa+2 \rho)+\lambda+\omega+\theta)(\eta(\rho+\theta)+\lambda(\eta+\theta))}{4 \eta(\rho+\theta)}
$$

This is only plausible if

$$
\lambda>\frac{(\alpha+\eta+3(\gamma+\phi)+2(\kappa+2 \rho)+\lambda+\omega+\theta)(\eta(\rho+\theta)+\lambda(\eta+\theta)}{4 \eta(\rho+\theta))}
$$

as $N>1$ at all times. This can only happen however, if $\lambda$ is negative, so the endemic equilibrium is not valid in this case. because of this, the diseases will always die out for this model once $I$ reaches a point where

$$
\beta S<4 \beta I+\alpha+\eta+3 \gamma+2 \kappa+\lambda+\omega+3 \phi+4 \rho+\theta
$$




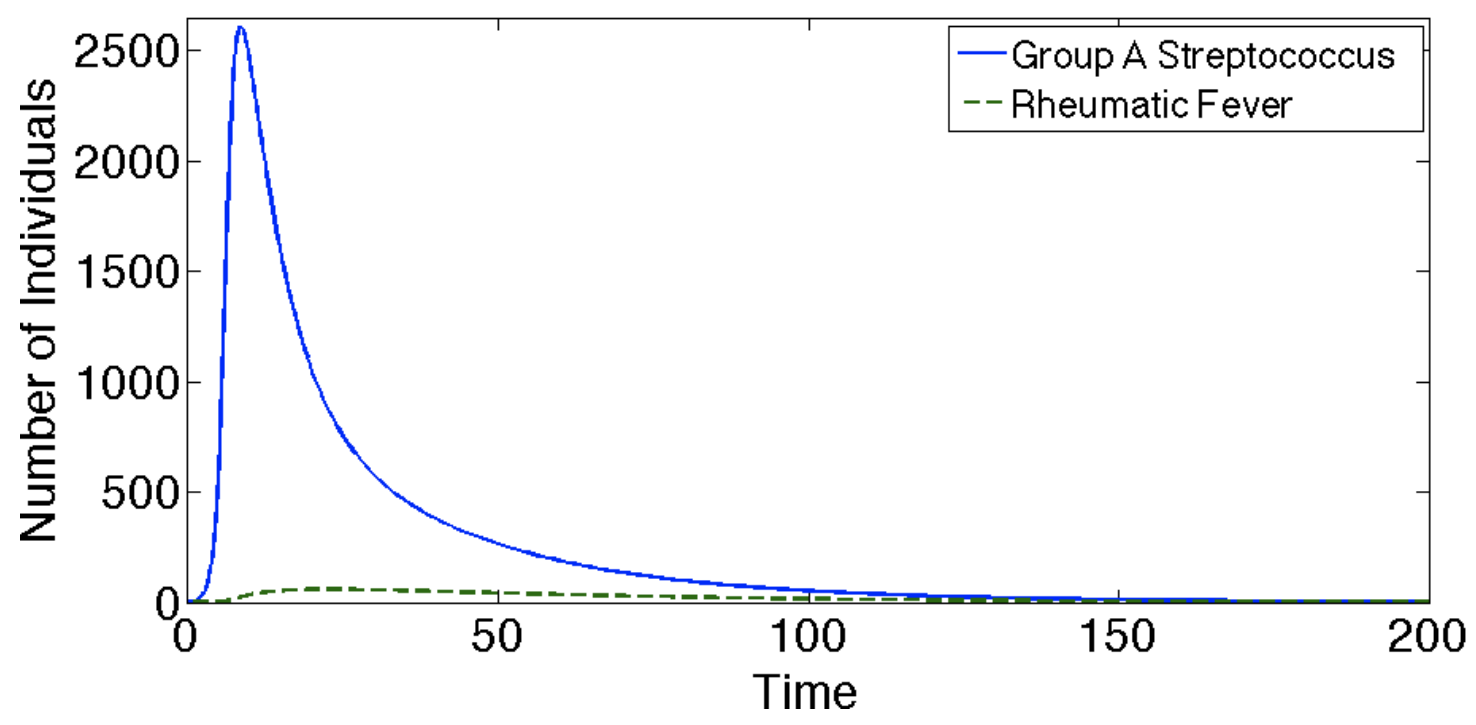

Figure 6.10: Plot of a numerical solution to the model seen in 6.9. Group A streptococcus numbers are represented by the solid line, acute rheumatic fever numbers are given by the dashed line. $\beta=0.00035, \gamma=0.1, \omega=0.006, \lambda=$ $0.3, \mu=0.01, \phi=0.1, \rho=0.1, \kappa=0.025, \eta=0.02, \alpha=0.003, \theta=0.001, N=$ 4000 .

Figure 6.10 shows a plot of the Group A streptococcus and acute rheumatic fever numbers over time for this model. For this plot, initially $\beta N>\alpha+\eta+3 \gamma+2 \kappa+$ $\lambda+\omega+3 \phi+4 \rho+\theta$ so the number of infectious individuals increases. With this increase in infected individuals and the consequential decrease in the number of those susceptible, we reach a point where

$$
\beta S<4 \beta I+\alpha+\eta+3 \gamma+2 \kappa+\lambda+\omega+3 \phi+4 \rho+\theta
$$

and the disease dies out. The parameters used in this plot are unrealistic, we are just using them to show an example of what might happen using this model. More accurate parameter values will be brought estimated in Chapter 7 .

\subsubsection{Age}

Group A streptococcus and acute rheumatic fever are both diseases that show increased prevalence among school aged children [12, 11, 24]. Because of this we 
should consider splitting the population into subgroups based on age. Something else we need to note, especially when we are using vital dynamics, is that individuals increase in age over time. We need to allow for individuals to change subgroups as they age. We will cover the inclusion of age and age groups in the model more in the next section.

\subsection{Using a recommended diagnosis and treat- ment algorithm to define risk groups}

The National Heart Foundation of New Zealand, together with the Cardiac Society of Australia and New Zealand, produced the New Zealand Guideline for Sore Throat Management in 2006. Figure 6.11 shows the flow chart based on the algorithm given in the guideline, this was produced in 2007. If we base our population groupings on the risk factors presented in this algorithm then we can treat each risk group as it is recommended a health professional should.

To keep the model slightly simpler for now, we will be focusing on age and ethnicity and acute rheumatic fever history. By ignoring geographic location we assume homogenous mixing of individuals in the population. This makes the model simpler but may affect accuracy.

\subsubsection{Risk Groups}

To make a model that covers age, ethnicity and acute rheumatic fever history we need to split each of the ethnic groups into age groups. We will include a few extra groups based on individual history of acute rheumatic fever and Group A streptococcus. These extra groups will start out empty and grow with time as individuals contract Group A streptococcus and develop acute rheumatic fever. In total we have nine subgroups of individuals.

Rates of movement between groups due to ageing are fixed. Everyone increases in age at the same rate.

Individuals under 3 years of age have a low risk of contracting Group A strepto- 
Algorithm: Guide for sore throat management

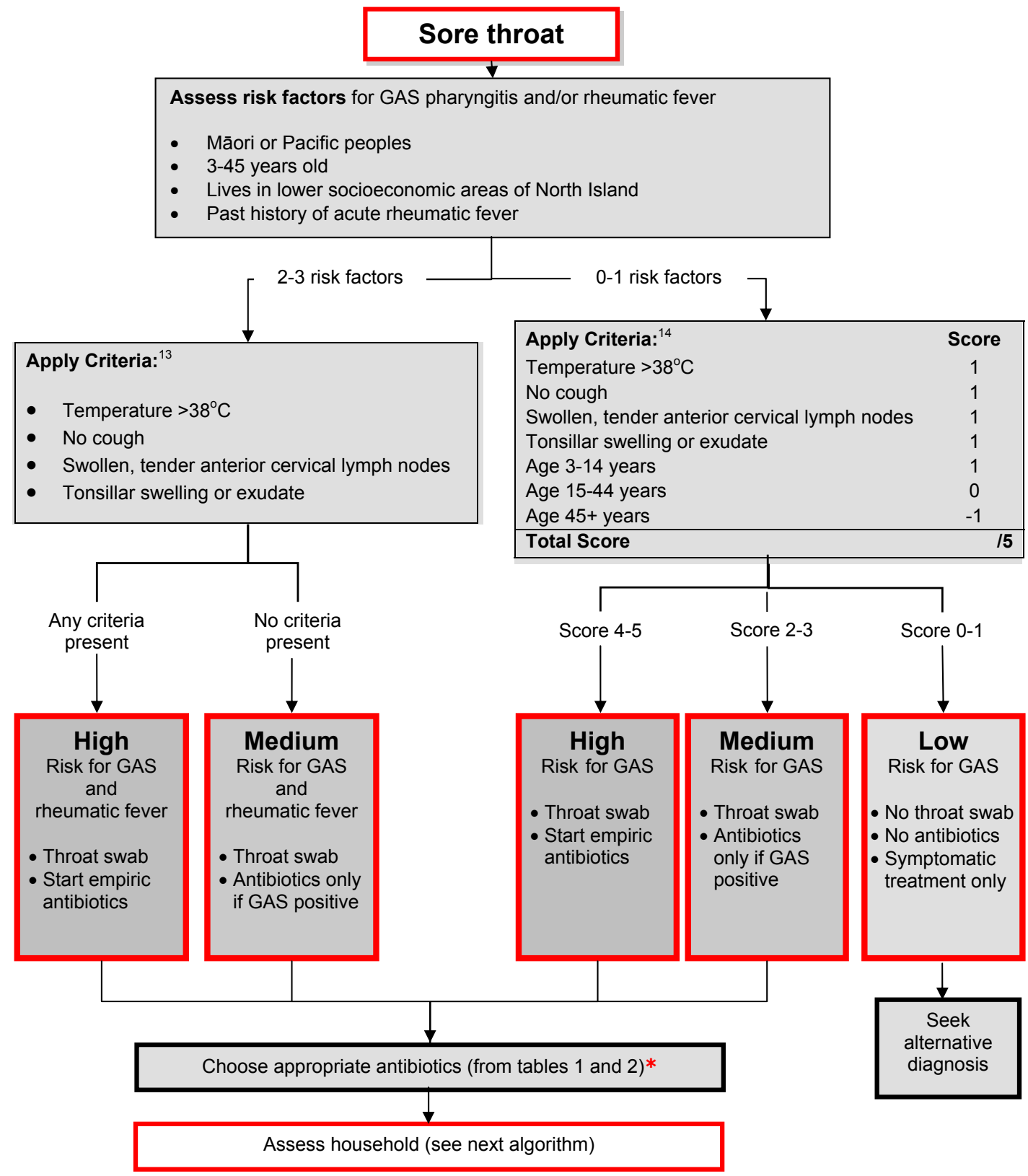

* If patient is on benzathine penicillin IM prophylaxis for acute rheumatic fever, and is GAS positive on throat swab, treat in the following way:

- If GAS positive in the first two weeks after IM penicillin injection has been given, treat with a 10 day course of erythromycin (see Table 3)

- If GAS positive in the $3^{\text {rd }}$ and $4^{\text {th }}$ weeks after IM penicillin injection, treat with a 10 day course of oral penicillin (see Table 3).

Sources:

Centor RM, Witherspoon JM, Dalton HP, Brody CE, Link K. Med Decis Making. (1:3) pp.239-246, copyright (c) 1981 by (Sage Publications Inc). Reprinted by Permission of SAGE Publications, Inc.

14 Copyright $\odot 2004$, American Medical Association, All rights reserved.

Figure 6.11: The algorithim from the New Zealand Guideline for Sore Throat Management. 
coccus or developing rheumatic fever [25]. Their mixing in the population is also limited. To keep things simpler we will not count individuals as being 'born' into the population until they turn 3. This also avoids the need to introduce a new subgroup or loop the age groups around, by including under threes in the same group with the over 45's.

An individual will spend 12 years in the under 15 age group, so the rate of movement out of this group is $\frac{1}{12 \text { years }}$ or $\frac{1}{4380}$ individuals per individual per day. Individuals spend 30 years in the 15 to 45 age group, so leave this group at a rate of $\frac{1}{10950}$ per day. Because we are assuming a constant birth rate that is equal to the rate of deaths, with no other sources of mortality, year groups are of equal size.

\subsubsection{Maori and Pacific Island Groups}

Maori and Pacific Islanders under 15 have the highest risk of developing acute rheumatic fever following a Group A streptococcus infection. This age group also has the highest risk of contracting a Group A streptococcus infection. We will label this group of individuals with the subscript $M 1$.

Maori and Pacific Island peoples between the ages of 15 and 45 inclusive have less risk of contracting Group A streptococcus than their younger counterparts. They still have a high risk of developing acute rheumatic fever after an infection however, if it should occur. We will give this group the subscript label M2.

Maori and Pacific Island peoples over the age of 45 have even less risk of contracting a Group A streptococcus infection. They also have a reduced risk of developing acute rheumatic fever when compared to those in the younger age groups. This group of individuals will be labeled with the subscript $M 3$.

\subsubsection{Other Ethnic Groups}

Other ethnicities have a reduced risk of developing acute rheumatic fever following a Group A streptococcus infection but their risk of this and Group A streptococcus still varies with age.

Individuals in other ethnicities under the age of 15 have the highest risk of con- 
tracting a Group A streptococcus infection, like Maori and Pacific Islanders in the same age group. Their risk of developing acute rheumatic fever following such an infection, however, is lower. The individuals in this subpopulation will be labeled with the subscript $E 1$.

Individuals of other ethnicities between the ages of 15 and 45 have less risk of contracting Group A streptococcus but their risk of developing acute rheumatic fever afterwards is still similar to the younger age group. This group of individuals will be referred to with the subscript $E 2$.

Individuals of other ethnicities over 45 have reduced risk of contracting a Group A streptococcus infection and a low likelihood of developing acute rheumatic fever after the infection. They will be labelled with the subscript E3.

\subsubsection{Rheumatic Fever History Groups}

As individuals move through the compartments and develop or don't develop acute rheumatic fever after Group A streptococcus infections, we can introduce subgroups based on acute rheumatic fever history.

Individuals who develop acute rheumatic fever after a case of Group A streptococcus recover into a separate group of susceptible individuals and become part of a subgroup we will label with the subscript $H$. This subgroup has increased risk of developing acute rheumatic fever if they do not receive treatment for a Group A streptococcus infection. Their history of having had acute rheumatic fever increases their likelihood of getting it again [27].

Individuals who recover from a Group A streptococcus infection without treatment and do not develop acute rheumatic fever afterwards may have a reduced susceptibility to developing acute rheumatic fever. We move these individuals into a subgroup labelled with the subscript $G$, as they recover from their Group A streptococcus infection. This subgroup works a bit like a buffer. It can be used to confirm that an individual really does have a low susceptibility to acute rheumatic fever. If individuals in this subgroup are able to recover from a Group A streptococcus infection without developing acute rheumatic fever, we assume their risk is low. 


\begin{tabular}{|c|c|c|c|c|c|c|}
\hline Group & GAS Rank & ARF Rank & GAS & ARF & Recovery & Treatment \\
\hline & & & $S \longrightarrow I$ & $I \longrightarrow A$ & $I \longrightarrow S$ & $I \longrightarrow T$ \\
\hline M1 & 2 & 2 & $\beta$ & $\omega$ & $\sigma$ & $r$ \\
\hline M2 & 1 & 2 & $\kappa$ & $\omega$ & $\sigma$ & $\theta$ \\
\hline M3 & 0 & 1 & $\gamma$ & $\alpha$ & $\lambda$ & $\theta$ \\
\hline E1 & 2 & 1 & $\beta$ & $\alpha$ & $\lambda$ & $\theta$ \\
\hline E2 & 1 & 1 & $\kappa$ & $\alpha$ & $\lambda$ & $\theta$ \\
\hline E3 & 0 & 0 & $\gamma$ & $\varphi$ & $\chi$ & $q$ \\
\hline H & 2 & 2 & $\beta$ & $\omega$ & $\sigma$ & $r$ \\
\hline G & 1 & 1 & $\kappa$ & $\alpha$ & $\lambda$ & $\theta$ \\
\hline L & 1 & 0 & $\kappa$ & $\varphi$ & $\chi$ & $q$ \\
\hline
\end{tabular}

Table 6.2: Table showing each subgroup's risk of Group A streptococcus (GAS) and acute rheumatic fever (ARF), and the parameters for the rates of movement into and out of the infectious compartment.

Individuals who start in a group with low risk of Group A streptococcus or acute rheumatic fever who contract Group A streptococcus though do not develop acute rheumatic fever after the infection move into a subgroup with low acute rheumatic fever risk, upon recovery. This subgroup we will label with the subscript $L$. Individuals can only move into this subgroup from groups with the subscript $G$ or E3.

\subsubsection{Creating the Model}

We can rank each risk group on their risk of contracting Group A streptococcus and developing acute rheumatic fever. Table 6.2 shows each subgroup and their risk of Group A streptococcus and acute rheumatic fever given a number, 0, 1 or 2. 2 indicates a high risk and 0 a low risk, but not no risk. 1 indicates a medium level risk.

If an individual is in the subgroup $L$ and develops acute rheumatic fever, upon recovery their risk of acute rheumatic fever is increased. Because of this increase they should change subgroups. They will become part of subgroup M3 upon recovery. This group has the same risk level for acute rheumatic fever, and because 
M3 is part of the oldest age group it cannot be aged out of. This is a modelling convenience, individuals cannot in reality change ethnicity. Even if the individual is not of Maori or Pacific Island ethnicity, they should be treated the same with regards to rheumatic fever. This gives the individual time to re-establish their low risk status or to move into the high risk subgroup $H$. A more detailed model would keep these ethnic groups separate and maybe use a new subgroup fro the from $L$ who develop acute rheumatic fever. Because their characteristics, relative to Group A streptococcus and acute rheumatic fever are very similar however, combing these subgroups does not greatly affect our model.

The flow of individuals between groups and subgroups is depicted in figure 6.12. Each sub group contains 3 or 4 compartments, so we have 29 compartments in total for this model. Each compartment needs an equation in the system. Because of varying rates of infection and recovery among the different subgroups, each requires a different combination of parameters. For this particular model we are using 19 different parameters, including those for ageing between susceptible compartments. 


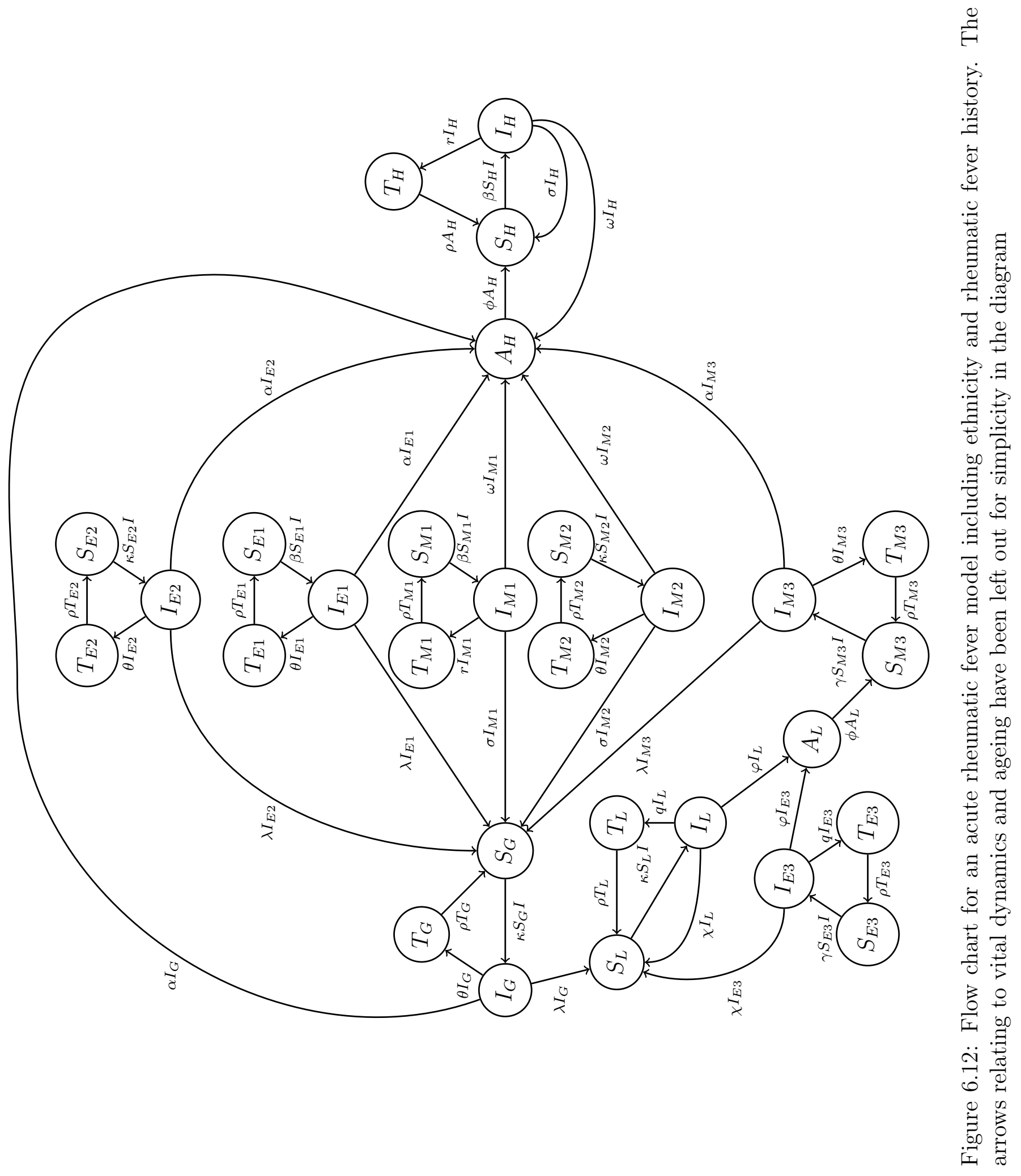


These equations can be seen in 6.10 below.

$$
\begin{aligned}
& \frac{d S_{M 1}}{d t}=M \mu-\beta S_{M 1} I+\rho T_{M 1}-\mu S_{M 1}-\frac{S_{M 1}}{4380} \\
& \frac{d I_{M 1}}{d t}=\beta S_{M 1}(I)-I_{M 1}(\omega+\sigma+r+\mu) \quad \frac{d T_{M 1}}{d t}=r I_{M 1}-(\rho+\mu) T_{M 1} \\
& \frac{d S_{M 2}}{d t}=\frac{S_{M 1}}{4380}-\kappa S_{M 2} I+\rho T_{M 2}-\mu S_{M 2}-\frac{S_{M 2}}{10950} \\
& \frac{d I_{M 2}}{d t}=\kappa S_{M 2}(I)-I_{M 2}(\omega+\sigma+\theta+\mu) \\
& \frac{d S_{M 3}}{d t}=\frac{S_{M 2}}{10950}-\gamma S_{M 3}(I)+\rho T_{M 3}+\phi A_{L}-\mu S_{M 3} \\
& \frac{d I_{M 3}}{d t}=\gamma S_{M 3}(I)-I_{M 3}(\alpha+\lambda+\theta+\mu) \\
& \frac{d S_{E 1}}{d t}=E \mu-\beta S_{E 1}(I)+\rho T_{E 1}-\mu S_{E 1}-\frac{S_{E 1}}{4380} \\
& \frac{d I_{E 1}}{d t}=\beta S_{E 1}(I)-I_{E 1}(\alpha+\lambda+\theta+\mu) \\
& \frac{d S_{E 2}}{d t}=\frac{S_{E 1}}{4380}-\kappa S_{E 2}(I)+\rho T_{E 2}+\phi A_{L}-\frac{S_{E 2}}{10950} \\
& -\mu S_{E 2} \\
& \frac{d I_{E 2}}{d t}=\kappa S_{E 2}(I)-I_{E 2}(\alpha+\lambda+\theta+\mu) \\
& \frac{d S_{E 3}}{d t}=\frac{S_{E 2}}{10950}-\gamma S_{E 3}(I)+\rho T_{E 3}-\mu S_{E 3} \\
& \frac{d I_{E 3}}{d t}=\gamma S_{E 3}(I)-I_{E 3}(\chi+\varphi+q+\mu) \\
& \frac{d A_{H}}{d t}=\omega\left(I_{M 1}+I_{M 2}+I_{H}\right) \\
& +\alpha\left(I_{M 3}+I_{E 1}+I_{E 2}+I_{G}\right)-(\phi+\mu) A_{H} \\
& \frac{d S_{H}}{d t}=\phi A_{H}-\beta S_{H} I+\rho T_{H}+\sigma I_{H}-\mu S_{H} \\
& \frac{d I_{H}}{d t}=\beta S_{H} I-I_{H}(r+\sigma+\omega+\mu) \\
& \frac{d S_{G}}{d t}=\sigma\left(I_{M 1}+I_{M 2}\right)+\lambda\left(I_{M 3}+I_{E 1}+I_{E 2}\right) \\
& +\rho T_{G}-\kappa S_{G} I-\mu S_{G} \\
& \frac{d I_{G}}{d t}=\kappa S_{G} I-I_{G}(\alpha+\theta+\lambda+\mu) \\
& \frac{d S_{L}}{d t}=\chi\left(I_{E 3}+I_{L}\right)+\lambda I_{G}-\kappa S_{L} I+\rho T_{L}-\mu S_{L} \\
& \frac{d I_{L}}{d t}=\kappa S_{L} I-(\varphi+\chi+q+\mu) I_{L} \\
& \frac{d A_{L}}{d t}=\varphi\left(I_{E 3}+I_{L}\right)-(\phi+\mu) A_{L} \\
& \frac{d T_{M 2}}{d t}=\theta I_{M 2}-(\rho+\mu) T_{M 2} \\
& \frac{d T_{M 3}}{d t}=\theta I_{M 3}-(\rho+\mu) T_{M 3} \\
& \frac{d T_{E 1}}{d t}=\theta I_{M 1}-(\rho+\mu) T_{E 1} \\
& \frac{d T_{E 2}}{d t}=\theta I_{E 2}-(\rho+\mu) T_{E 2} \\
& \frac{d T_{E 3}}{d t}=q I_{E 3}-(\rho+\mu) T_{E 3} \\
& \frac{d T_{H}}{d t}=r I_{H}-(\rho+\mu) T_{H} \\
& \frac{d T_{G}}{d t}=\theta I_{G}-(\rho+\mu) T_{G} \\
& \frac{d T_{L}}{d t}=q I_{L}-(\rho+\mu) T_{L}
\end{aligned}
$$

$M$ and $E$ represent the proportions of Maori and Pacific Island peoples and other ethnicities respectively.

$\beta, \kappa$ and $\gamma$ represent the different levels of susceptibility to Group A streptococcus in each age group.

$\omega, \alpha$ and $\varphi$ represent the different rates of acute rheumatic fever development.

$\sigma, \lambda$ and $\chi$ represent the different rates of recovery from Group A streptococcus, 
back to being susceptible without passing through treatment.

$r, \theta$ and $q$ represent the different rates of treatment for Group A streptococcus in each risk group.

The rates for each subgroup can be seen in Table 6.2 ,

$\mu$ is still the birth/death rate and $\rho$ is the recovery rate following treatment for a Group A streptococcus infection.

\subsubsection{The Basic Reproduction Number}

Because of the complicated structure of this model, finding equilibriums points using the methods shown previously is difficult. We can however write an equation for the basic reproduction number $\mathcal{R}_{0}$. We can then use this value to help us see how the system may behave. By using the definition of $\mathcal{R}_{0}$ as the rate of infection multiplied by how long an individual is infectious for we can calculate $\mathcal{R}_{0}$ for our model.

Let $E$ be the total number of individuals being infected by individuals in compartment $I_{M 1}$ per unit of time. A fraction $\frac{\beta S_{M 1}}{E}$ move into the compartment $I_{M 1}$ as they become infectious. While individuals are in the compartment $I_{M 1}$ they are causing $E$ new infections per unit of time. The mean amount of time spent in compartment $I_{M 1}$ is $\frac{1}{r+\omega+\sigma+\mu}$. So the portion of the basic reproduction number due to $I_{M 1}$ is

$$
\mathcal{R}_{M 1}=\frac{\beta S_{M 1}}{E} \times \frac{E}{r+\omega+\sigma+\mu}=\frac{\beta S_{M 1}}{r+\omega+\sigma+\mu}
$$

We can do the same calculation for each infectious compartment and sum them up to find $\mathcal{R}^{*}$ as shown below.

$$
\begin{aligned}
\mathcal{R}^{*} & =\beta\left(\frac{S_{M 1}+S_{H}}{r+\omega+\sigma+\mu}+\frac{S_{E 1}}{\theta+\alpha+\lambda+\mu}\right) \\
& +\kappa\left(\frac{S_{M 2}}{\theta+\omega+\sigma+\mu}+\frac{S_{E 2}+S_{G}}{\theta+\alpha+\lambda+\mu}+\frac{S_{L}}{q+\chi+\varphi+\mu}\right) \\
& +\gamma\left(\frac{S_{M 3}}{\theta+\alpha+\lambda+\mu}+\frac{S_{E 3}}{q+\chi+\varphi+\mu}\right)
\end{aligned}
$$


This is the same expression as that given by the largest eigenvalue of the next generation matrix.

$\mathcal{R}_{0}$ is $\mathcal{R}^{*}$ at time zero. At the start of an outbreak compartments $A, G$ and $L$ are empty, so

$$
\begin{aligned}
\mathcal{R}_{0} & =\beta\left(\frac{S_{M 1}}{r+\omega+\sigma+\mu}+\frac{S_{E 1}}{\theta+\alpha+\lambda+\mu}\right) \\
& +\kappa\left(\frac{S_{M 2}}{\theta+\omega+\sigma+\mu}+\frac{S_{E 2}}{\theta+\alpha+\lambda+\mu}\right) \\
& +\gamma\left(\frac{S_{M 3}}{\theta+\alpha+\lambda+\mu}+\frac{S_{E 3}}{q+\chi+\varphi+\mu}\right)
\end{aligned}
$$




\section{Chapter 7}

\section{Introducing Real Data and Conclusions}

If we are going to make a model to simulate disease in a real population, we need to use parameter values that apply to the population and that disease. We can use the information about acute rheumatic fever and Group A streptococcus, seen in Chapter 3 to estimate parameters such as recovery rate with and without treatment, and rates of acute rheumatic fever development. Parameters such as population size, proportions of ethnicities and birth/death rates we can find through Statistics New Zealand. We may have to modify some parts of our model to fit what data may be available.

Using data on births and deaths from the Statistics New Zealand, we estimate a value of $\mu$. While birth rates are greater than death rates in New Zealand at the moment, we want to estimate a value somewhere between so we can use $\mu$ for both births and deaths. We will use $\mu=0.000027$ deaths per person per day $=$ births, based on tables from Statistics New Zealand [2] This does estimate a lifespan of about 100 years, but this is due to the birth rate being higher that the death rate in reality, and for the sake of simplicity in our model we are assuming no other sources of mortality aside from natural causes . Parameters are defined on rates of change per day. We will use a population size of $N=4,000,000$. 


\subsection{Ethnic Proportions}

Using data from the 2006 Census, provided by Statistics New Zealand we can estimate the proportions of Maori and Pacific peoples in each age group. Table 7.1 shows the proportions of the relevant ethnicities in each age group [2].

\begin{tabular}{|l|c|c|c|c|}
\hline Ethnicity & $\mathbf{0}$ to $\mathbf{1 4}$ & $\mathbf{1 5}$ to $\mathbf{4 4}$ & $\mathbf{4 5 +}$ & Total \\
\hline European and Other & $11.93 \%$ & $33.05 \%$ & $32.23 \%$ & $79.36 \%$ \\
\hline Maori and Pacific & $6.2 \%$ & $9.29 \%$ & $3.89 \%$ & $20.64 \%$ \\
\hline Total & $18.12 \%$ & $42.34 \%$ & $36.12 \%$ & $100 \%$ \\
\hline
\end{tabular}

Table 7.1: Percentage of the total population categorised by age and ethnicity

\subsection{Rates of Rheumatic Fever Development}

The parameters $\omega, \alpha$ and $\varphi$, are used to represent the different rates of rheumatic fever development in each group. Several factors can affect this rate. We need to know how long it takes acute rheumatic fever to develop after a Group A streptococcus infection and the rate of acute rheumatic fever in that group. We know from Chapter 3 that it takes about 3 weeks for an individual to start showing symptoms of acute rheumatic fever following a Group A streptococcus infection. If we treat this as 20 days we can estimate the rate of acute rheumatic fever development for individuals susceptible to acute rheumatic fever as 0.05 per day. $\alpha$ represents the rate of acute rheumatic fever for groups $M 3, E 1, E 2$ and $G$. From Section 3.2 we can estimate that approximately $3 \%$ of individuals in these groups are likely to develop acute rheumatic fever following an untreated Group A streptococcus infection. Because of this we will estimate $\alpha=0.03 \times 0.05=0.0015$ per person per day.

$\omega$ represents the rate of acute rheumatic fever development for individuals in groups $M 1, M 2$, and $H$. That is Maori and Pacific peoples under 40 and individuals with a history of acute rheumatic fever. The upper age limit of group 2 has been modified to 40 because of data availability in age group distribution. We saw in section 3.2 
that individuals with a history of acute rheumatic fever have an increased chance of $30 \%$ to $80 \%$ of recurrence following another Group A streptococcus infection. We also saw that Maori and Pacific peoples are 22 to 75 times more likely to develop acute rheumatic fever than New Zealand Europeans. From this information we could estimate $22 \times 3 \%=66 \%$ to over $100 \%$. But with an increased likelihood of treatment for these groups we can reduce this a bit, to about $50 \%$. We can then estimate $\omega=0.5 \times 0.05=0.0215$ per person per day.

$\varphi$ is the rate of acute rheumatic fever development for those with a low risk. That is those in group E3 and group $L .0 .3 \%$ is the low end of the range given in section 3.2 for the general population. This means we can estimate $\varphi=0.003 \times 0.05=0.00015$ per person per day.

\subsection{Rates of Treatment and Recovery}

Rates of treatment are something we can vary to try and reduce numbers of acute rheumatic fever and Group A streptococcus. The part that we can't vary is how long it takes for individuals to receive treatment. There is a window of about 9 days within which an individual can receive treatment to prevent acute rheumatic fever [4]. Symptoms for Group A streptococcus usually show up within 3 days and then disappear within 4 more. We work with 5 days as it is in the middle and gives us a nice value of 0.2 to multiply the proportions of individuals receiving treatment by. We will start with all groups receiving treatment equally at at a proportion of $20 \%$. $r=\theta=q=0.2 \times 0.2=0.04$ per person per day.

If an individual with Group A streptococcus goes untreated, the infection can last for 7 to 10 days [29]. After this they will either develop acute rheumatic fever or recover. The rate of recovery if untreated is 0.1 multiplied by the proportion of individuals likely to recover. For groups $M 1, M 2$ and $H$ we have $\sigma=0.1 \times 0.1=$ 0.01 per person per day. For groups $E 3$ and $L$, we will use $\chi=0.8 \times 0.1=0.08$ per person per day. For all the other groups we use $\lambda=0.65 \times 0.1=0.065$ per person per day.

Treatment of Group A streptococcus reduces the duration of symptoms, and in 
our model eliminates the risk of developing rheumatic fever. The duration of symptoms is reduced by 1 to 2 days but 10 days of treatment with antibiotics is recommended for routine strep throat treatment [21]. So an individual will spend 10 days in the treatment compartment then recover to the relevant susceptible compartment, implying $\rho=0.1$ per person per day.

Following treatment for rheumatic fever symptoms, the attacks usually cease within 2 month of the initial infection. This is about 60 days minus the time spent in a Group A streptococcus compartment, which is about about 20 days if no treatment was received. So we have $\phi=0.025$ per person per day.

\subsection{Rates of Infection}

Because of the lack of data on strep throat these values are the hardest to estimate. We need to work backwards from numbers of rheumatic fever cases. We can use the rates of development that we know and simpler models to try to estimate what the overall rate of infection is, then we need to take a bit of an educated guess as to the individual values of $\beta, \kappa$ and $\gamma$. Using data from the New Zealand Public Health observatory [23], we can estimate an average of 9.3 cases of acute rheumatic fever each month from 1997 to 2010. that is 0.31 cases per day.

We also know $\beta>\kappa>\gamma>0$, the majority of cases occur in the under 15 age group. For now we will start with $70 \%$ of infection occurring in this age group. $20 \%$ for age group 2 and $10 \%$ for age group 3 .

We know that rheumatic fever rates are not dropping, so $\mathcal{R}^{*} \geq 1$. They are not growing hugely either so the value must still be close to 1 . Using equation 6.11 with our current parameters we get

$$
\begin{aligned}
\mathcal{R}_{0} & =\beta\left(\frac{0.062 N}{0.04+0.0215+0.01+0.000027}+\frac{0.1193 N}{0.04+0.0015+0.065+0.000027}\right) \\
& +\kappa\left(\frac{0.0929 N}{0.04+0.0215+0.01+0.000027}+\frac{0.3305 N}{0.04+0.0015+0.065+0.000027}\right)
\end{aligned}
$$




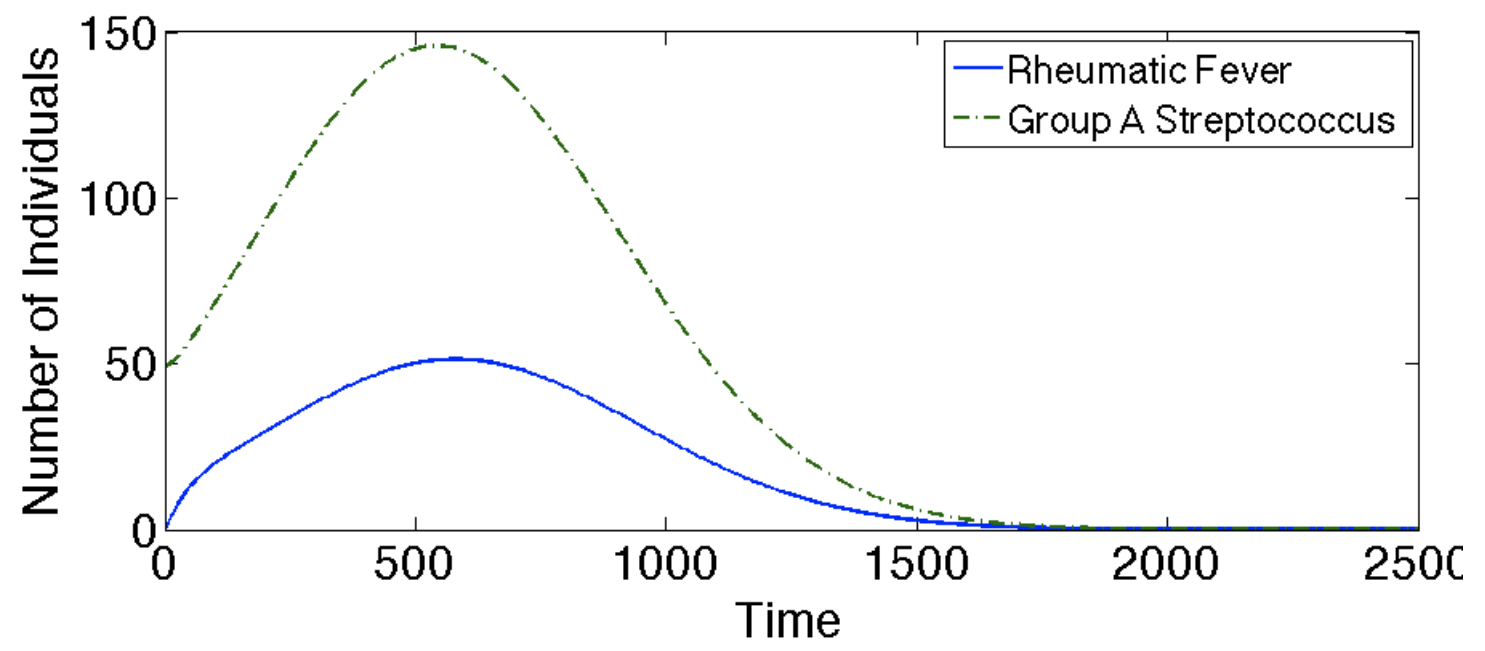

Figure 7.1: Plot of a numerical solution for the model described in 6.10 and Figure 6.12. Group A streptococcus numbers are represented by the dashed line and acute rheumatic fever numbers by the solid line. The parameter values are defined in the above sections.

$$
\begin{aligned}
+\gamma\left(\frac{0.0389 N}{0.04+0.0015+0.065+0.000027}+\frac{0.3223 N}{0.04+0.08+0.00015+0.000027}\right) \\
\mathcal{R}_{0}=\beta(0.8668+1.1199) N+\kappa(1.2988+3.1025) N+\gamma(0.3652+2.6819) N \\
\quad=1.9868 \beta N+4.4013 \kappa N+3.0471 \gamma N
\end{aligned}
$$

$\mathcal{R}^{*}=1$ is where our endemic equilibrium would be. For now we can use $\mathcal{R}_{0}=1$ can give us a good estimate of what $\beta, \kappa$ and $\gamma$ should be. If we use our ratios from earlier of $70 \%, 20 \%$ and $10 \%$ of cases in each age group, we can estimate $\beta, \kappa$ and $\gamma$ using, for example, $\beta N=0.7 \mathcal{R}_{0}$. Setting $\mathcal{R}_{0}=1$ we get;

$$
\beta=6.93 \times 10^{-8}, \quad \kappa=1.32 \times 10^{-8} \text { and } \gamma=0.73 \times 10^{-8}
$$

A plot of numerical solutions for the model given in 6.10 using these parameters is shown in Figure 7.1 In this case there is an initial spike but then the disease dies out. $\mathcal{R}_{0}=0.9923<1$ in this case however due to rounding errors in estimating $\beta, \kappa$ and $\gamma$. So the disease is dying out when we would expect it to. If we round 


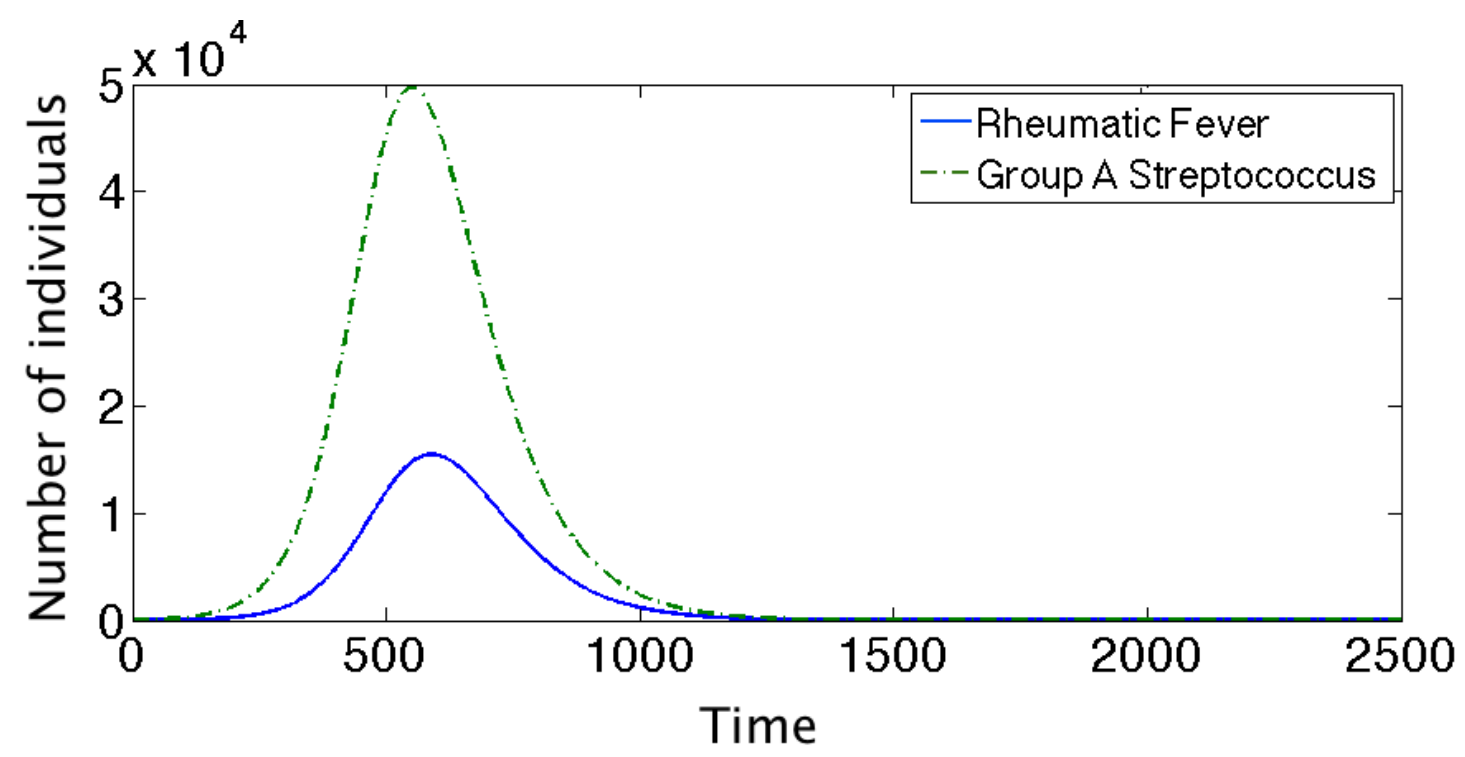

Figure 7.2: Numerical solutions for the model in 6.10. Group A streptococcus numbers a represented by the dashed line and acute rheumatic fever numbers by the solid line. The parameter values are those defined in the above sections. $\beta=7 \times 10^{-8}, \kappa=2 \times 10^{-8}$ and $\gamma=1 \times 10^{-8}$.

up to

$$
\beta=7 \times 10^{-8}, \kappa=2 \times 10^{-8} \text { and } \gamma=1 \times 10^{-8}
$$

We get $\mathcal{R}_{0}=1.1474>1$. Figure 7.2 shows what happens with these infection rate changes. The peak at the beginning is a lot steeper but the disease is still dying out. Figure 7.3 shows how the reproduction number is changing over this time. $\mathcal{R}_{0}$, is the initial reproduction number at time 0 . Initially the reproduction number is greater than 1 and the number of infected individuals increases, but as the number of susceptible individuals gets smaller so does the effective reproduction number. When we reach the point where $\mathcal{R}^{*}<1$ the disease starts to die out. The model with these parameters has no stable endemic equilibrium.

In this model there appears to be a threshold for $\mathcal{R}^{*}$ to stabilise. Keeping the $70 \%$, $20 \% 10 \%$ for proportion of cases in age groups, the reproduction number starts to settle around 1 when we start with $\mathcal{R}_{0}$ at about 1.7 . this is with

$$
\beta=1.123 \times 10^{-7}, \kappa=2.135 \times 10^{-8} \text { and } \gamma=1.185 \times 10^{-8}
$$




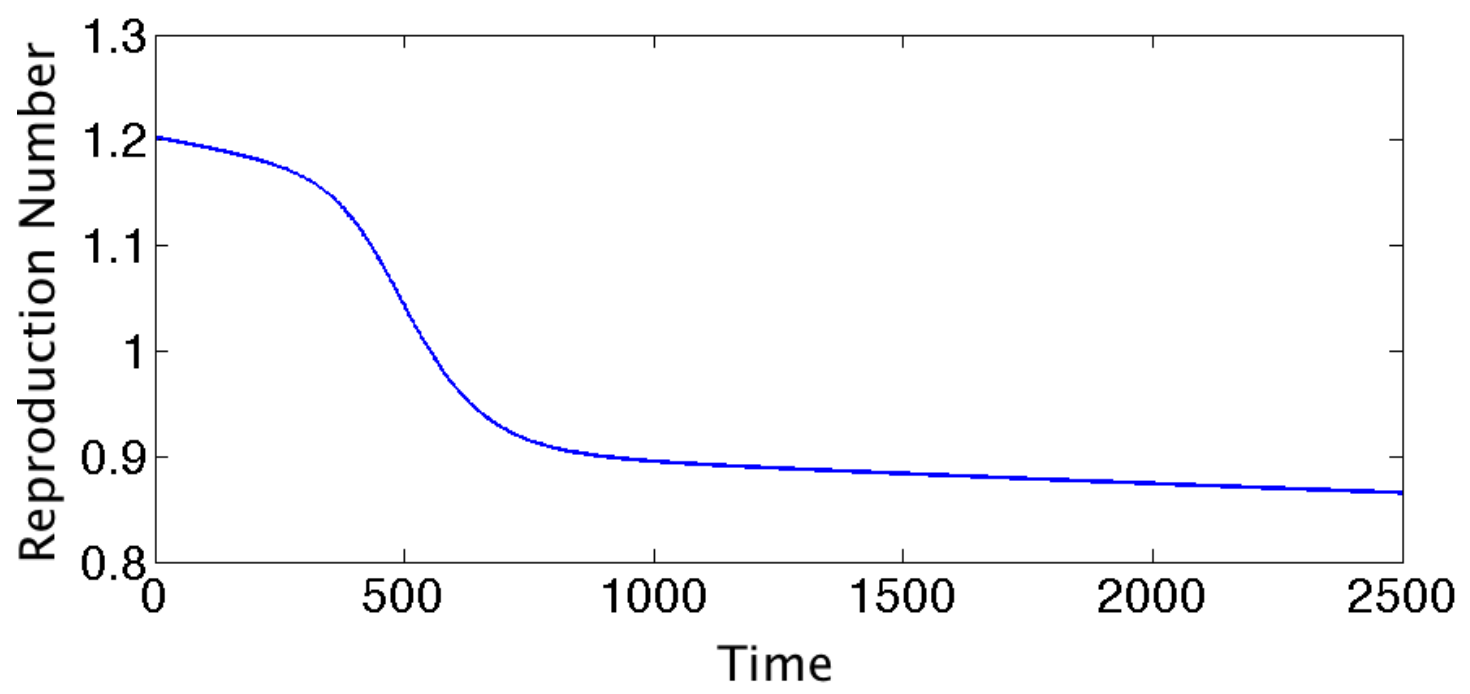

Figure 7.3: The effective reproduction number, given by the numerical solution to the model in 6.10 using the same parameters as the plot in figure 7.2 .

Figure 7.4 shows the effective reproduction number over time using these values.

If we play with these infection rate parameters some more and just use $\beta=7 \gamma, \kappa=$ $2 \gamma$, The numbers of infected and those with rheumatic fever, start to settle out with an $\mathcal{R}_{0}$ of about 1.68. Figure 7.5 shows a plot of this using $\gamma=1.4 \times 10^{-8}$.

We can reduce $\gamma$ to about $1.345 \times 10^{-8}$ before Group A streptococcus and acute rheumatic fever start dying out instead of settling towards an equilibrium. Figure 7.6 shows a plot for this value of $\gamma$.

\subsection{Matching Acute Rheumatic Fever Data}

In terms of acute rheumatic fever numbers, we are aiming for them to settle at around 12 individuals with acute rheumatic fever at any point in time. We are currently not accounting for seasonality in this model. This is based on the data from Statistics New Zealand with about 0.31 new cases per day and each case taking about a month to recover. The number of individuals with acute rheumatic fever this model, with the current parameter values is producing is still about 16 times too high. $\mathcal{R}_{0}=1.52$ with these infection rate values. We may need to modify 


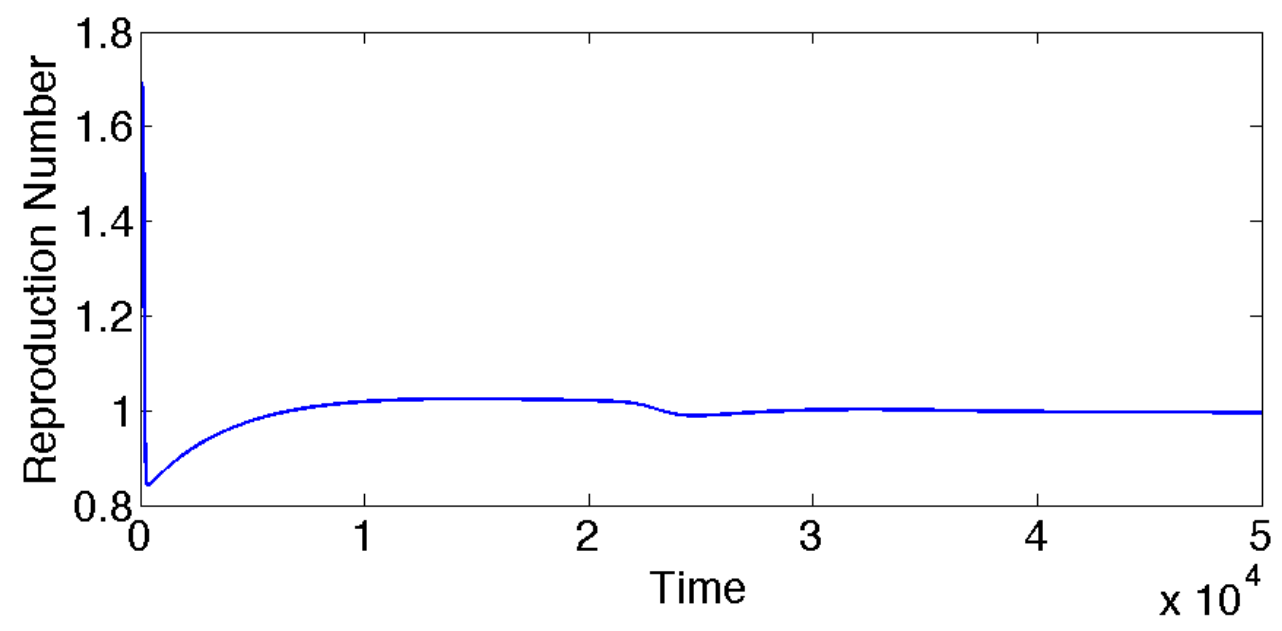

Figure 7.4: The effective reproduction number, given by the numerical solution to the model in 6.10 and using $\beta=1.123 \times 10^{-7}, \kappa=2.135 \times 10^{-8}$ and $\gamma=$ $1.185 \times 10^{-8}$, with the other parameter values as defined above.

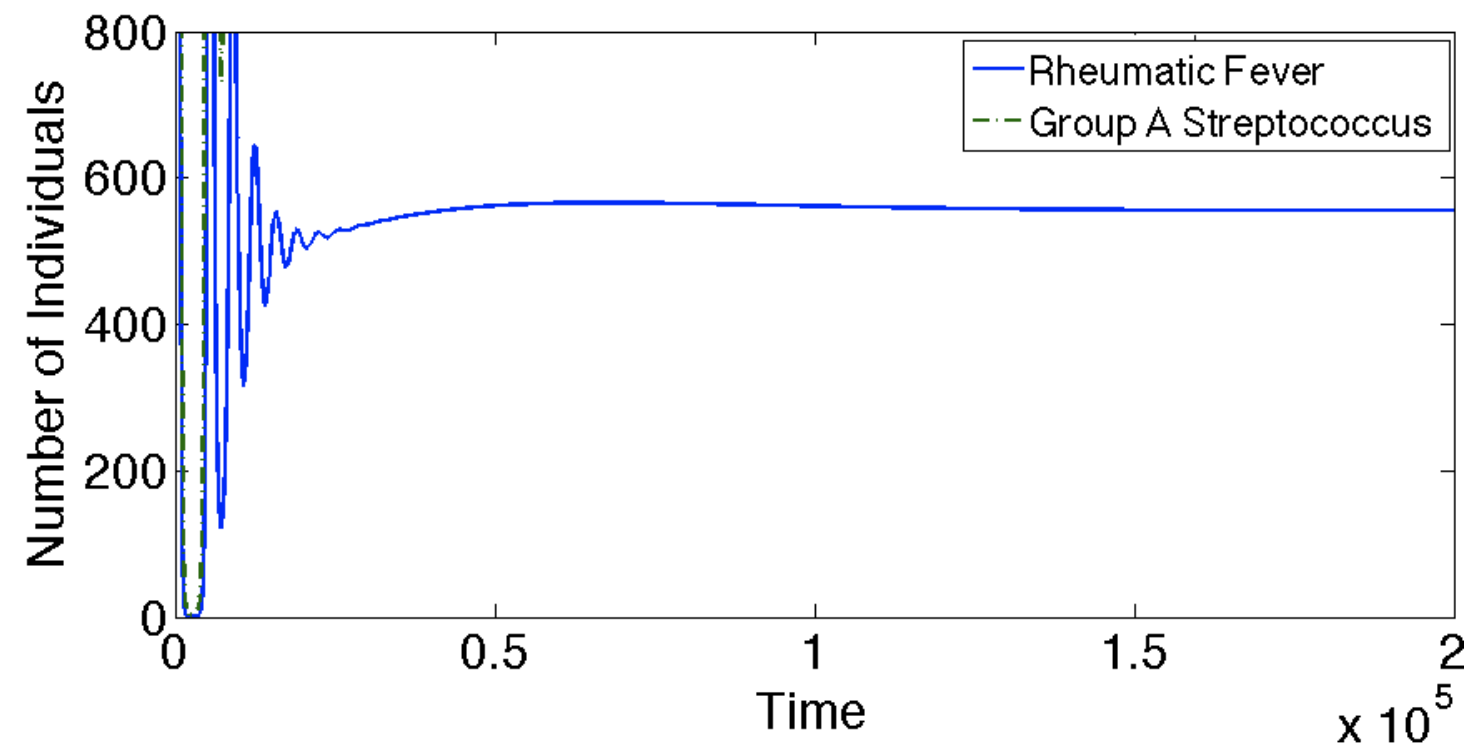

Figure 7.5: Acute rheumatic fever numbers given by the numerical solution to 6.10 with $\gamma=1.4 \times 10^{-8}$. 


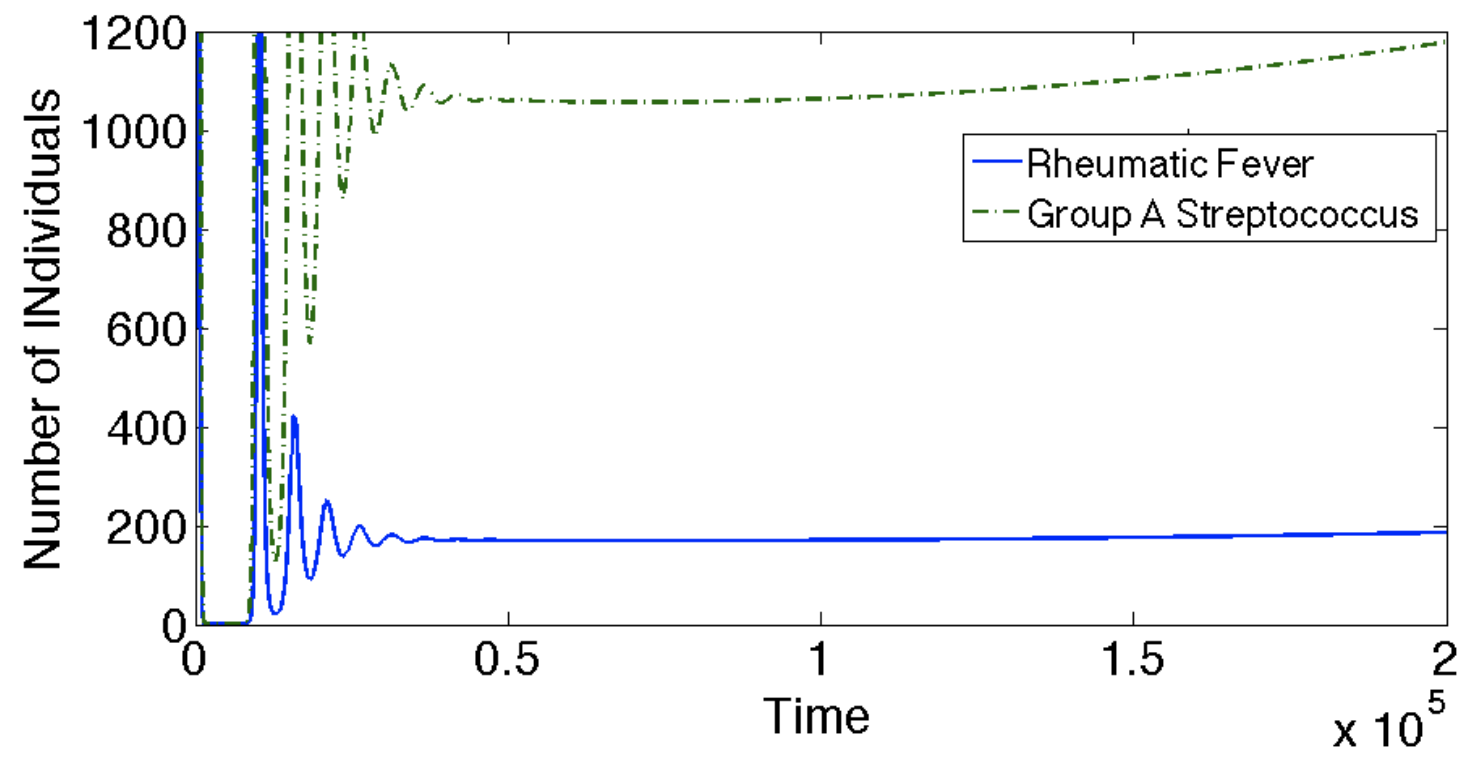

Figure 7.6: The solid line shows rheumatic fever numbers, given by the numerical solution to the model in 6.10 , with $\gamma=1.345 \times 10^{-8}$. The dashed line shows the number of individuals infected with Group A streptococcus.

some of the other parameters to achieve the correct rate of acute rheumatic fever. We can do this by reducing $\omega, \alpha$ and $\varphi$.

Figure 7.8 shows a plot where we have acute rheumatic fever numbers settling out to about 12 individuals. A small reduction in the rate of acute rheumatic fever development from that shown in Figure 7.6 actually increased acute rheumatic fever numbers. This may be because individuals spent longer in the infected compartment. Figure 7.7 shows what happens when make small reduction.

By reducing the acute rheumatic fever development rates we have increased the basic reproduction number to $\mathcal{R}_{0}=1.55$.

Comparing these rates to the original estimates made in Sections 7.2 and 7.4 , the largest change has been made to the rate at which individuals at high risk of developing acute rheumatic fever develop the disease following a Group A streptococcus infection, parameter $\omega$. In our original estimate in section 7.2 we had $\omega \approx 150 \varphi$ in our most recent estimate $\omega \approx 55 \varphi$ so this proportionality has been reduce by almost a third. In both estimates $\alpha=10 \varphi$, this has remained the same. There has beed a large reduction in the size of $\varphi$ between our original estimate and now, 


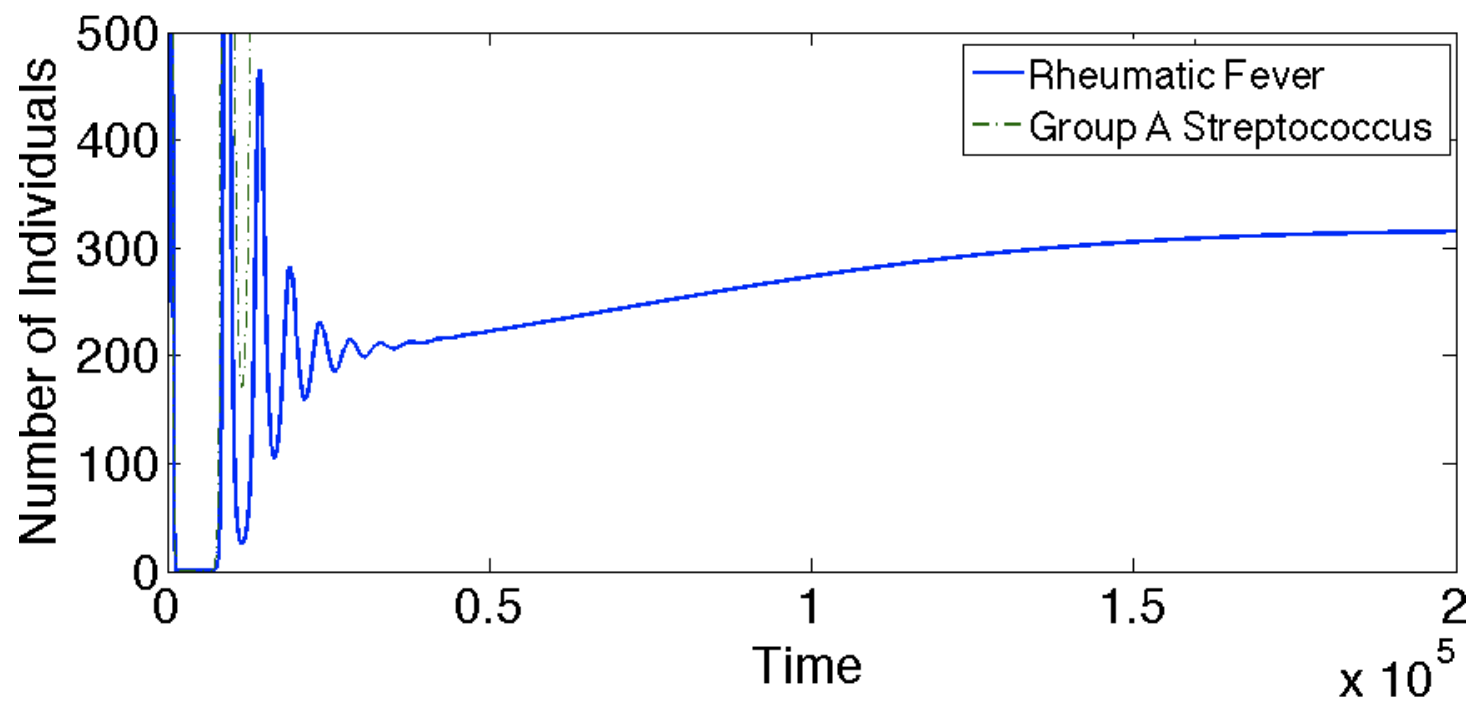

Figure 7.7: Rheumatic fever numbers, given by the numerical solution to 6.10 with $\gamma=1.345 \times 10^{-8}, \omega=0.021, \alpha=0.001$ and $\varphi=0.0001$.

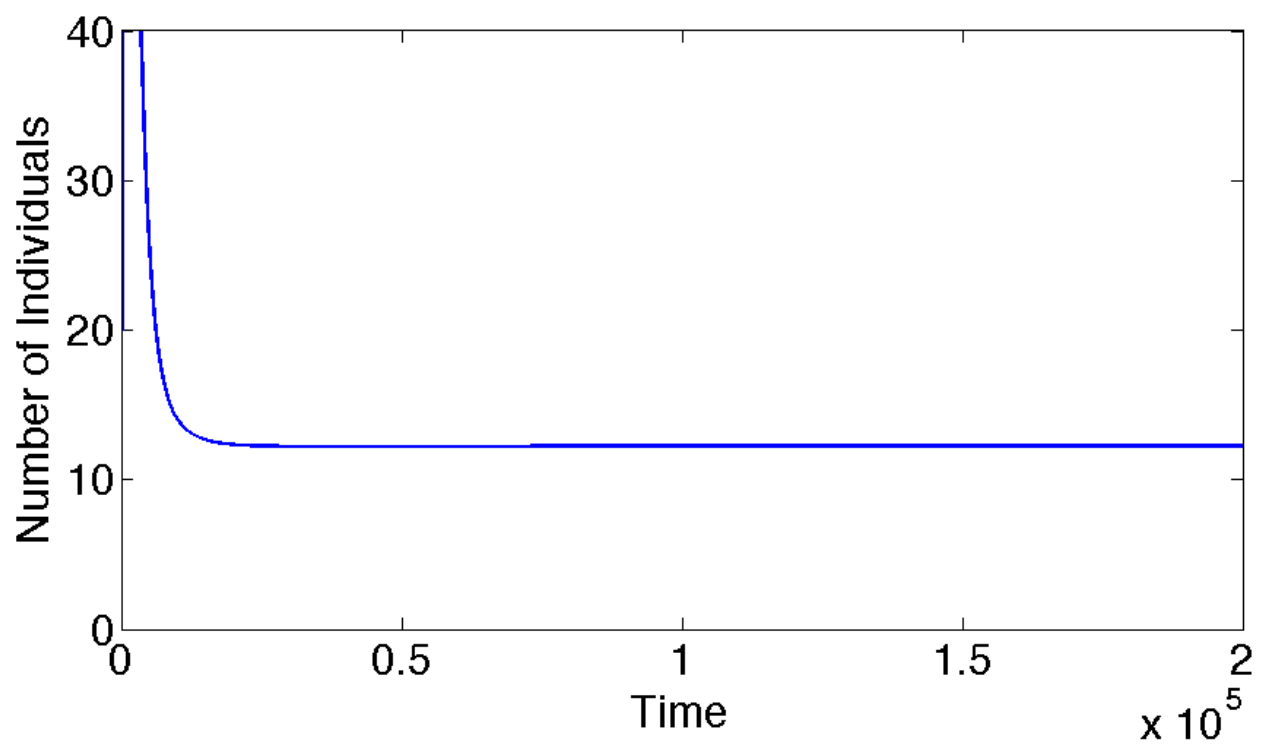

Figure 7.8: Acute rheumatic fever numbers, given by a numerical solution to the model in 6.10, with $\omega=0.000022, \alpha=0.000004$ and $\varphi=0.0000004$. 
from $\varphi=1.5 \times 10^{-4}$ to $\varphi=0.004 \times 10^{-4}$.

As far as rates of infection are concerned $\gamma$ is now slightly less than twice our original estimate, changing from $\gamma=0.73 \times 10^{-8}$ to $\gamma=1.345 \times 10^{-8}$. In terms of proportion $\kappa$ and $\beta$ have not changed too much. In section 7.4 we originally had $\kappa \approx 1.8 \gamma$ and $\beta \approx 9.5 \gamma$, we no have $\kappa=2 \gamma$ and $\beta=7 \gamma$. So the the relative parameters are proportionally similar to what we estimated in section 7.4 .

\subsection{Reducing Rheumatic Fever with Treatment of Strep Throat}

In the previous plots of the model, the treatment rate has been $0.04 I$ for all the subgroups. Keeping all the other above parameters the same we will try now varying treatment rates. First of all we will try focusing all the treatment on the groups with a high risk of rheumatic fever. Let

$$
r=0.2, \text { and } \theta=q=0
$$

The plot of this is shown in Figure 7.9. This focused method has actually increased rheumatic fever numbers. The basic reproduction number in this case becomes $\mathcal{R}_{0}=2.67$. So we can see just by $\mathcal{R}_{0}$ that we have made things worse.

Because any treatment plan would be implemented after we have reached equilibrium, for a disease like rheumatic fever that has been in the population for a while, we should try using the values of each compartment at equilibrium as our initial values. Exclusively focusing treatment on those most a risk of rheumatic fever still increases rheumatic fever numbers over all. The reproduction number now becomes $\mathcal{R}^{*}=2.86$ at the point in time we introduce exclusive treatment.

Maybe a better approach would be to proportionally focus treatment based on risk, but not be exclusive. Let us try

$$
r=0.1, \theta=0.06 \text { and } q=0.04
$$




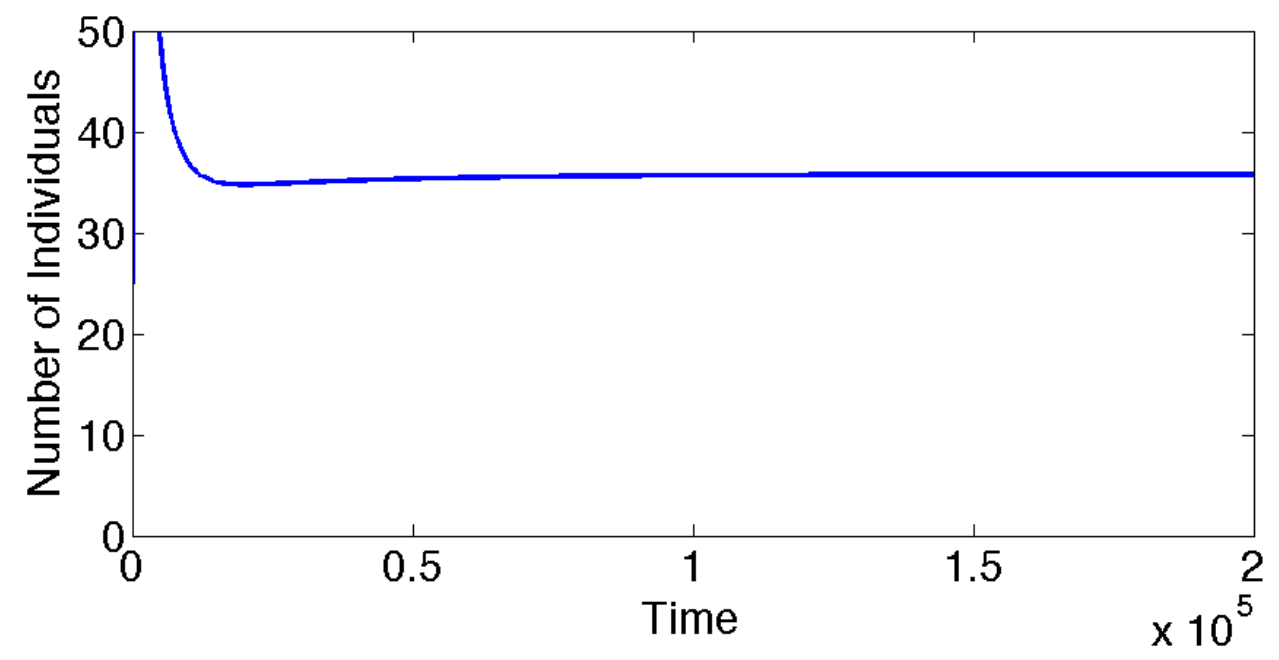

Figure 7.9: Rheumatic fever numbers given by the numerical solution to 6.10, with treatment rates $r=0.2$ and $\theta=q=0$. Infection rates and other parameters are the same as in figure 7.8 .

The plot using these treatment rates is shown in figure 7.10, This proportional method of treatment looks quite successful in reducing acute rheumatic fever numbers. Acute rheumatic fever has been reduced to an average of 1.4 individuals at any one time. In this case $\mathcal{R}^{*}$ is reduced to 0.92 . Figure 7.11 shows how the reproduction number changes over time, from the introduction of this proportional treatment.

\subsection{Conclusions}

Rates of acute rheumatic fever remain an issue in New Zealand. A large concern is the increased rates for Maori and Pacific peoples. While we have not covered the socio-economic or geographic distribution of acute rheumatic fever in this thesis, these are also issues which require attention.

We have developed a model which accounts for the different rates of Group A streptococcus and acute rheumatic fever among different age groups and ethnic- 


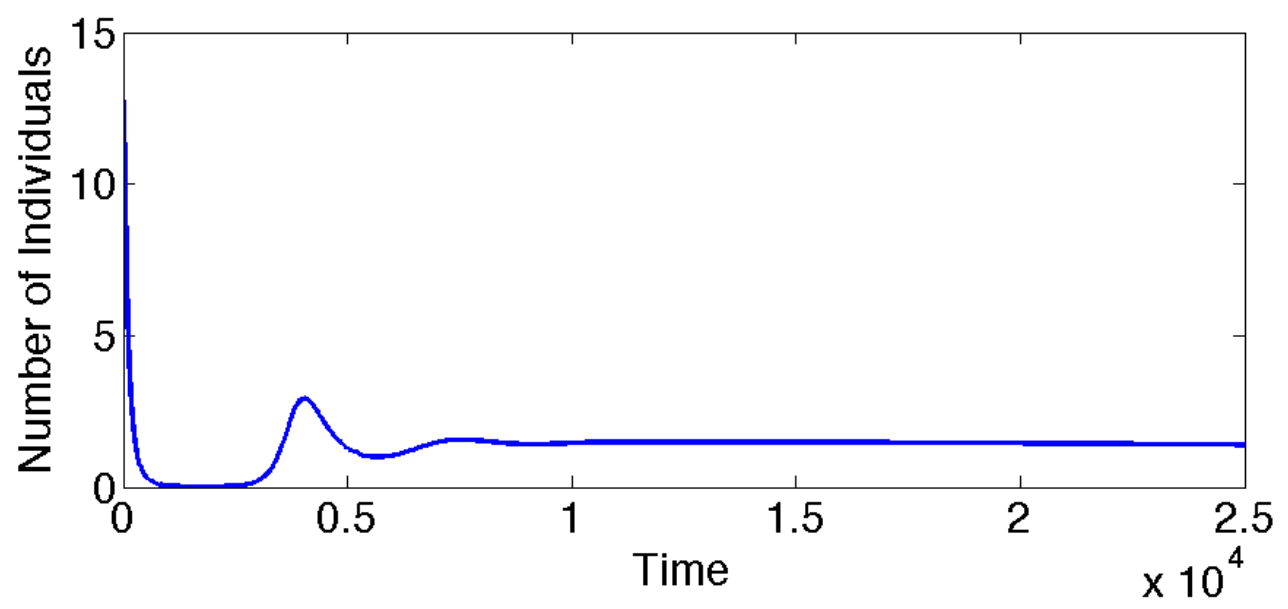

Figure 7.10: Acute rheumatic fever numbers, given by the numerical solution to 6.10, with treatment rates $r=0.1, \theta=0.6$ and $q=0$. All other parameters are the same as in figure 7.8 .

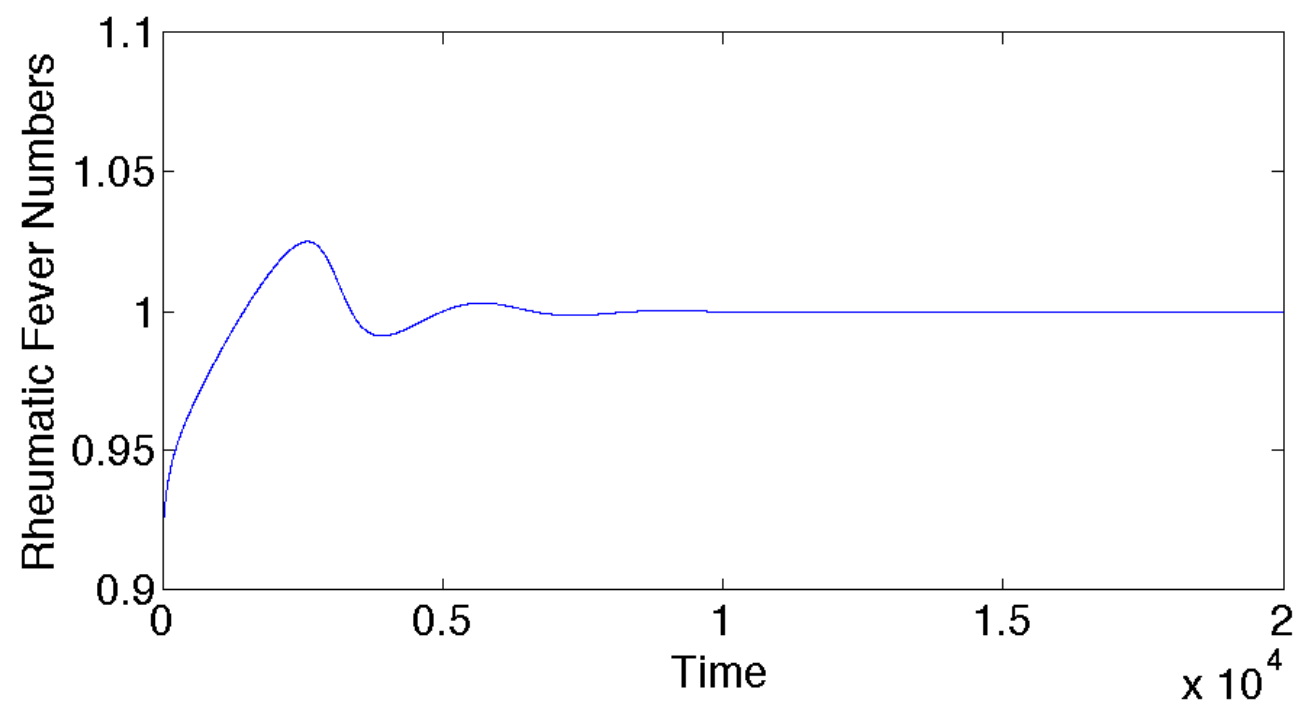

Figure 7.11: The reproduction number given by the numerical solution to 6.10 with treatment rates $r=0.1, \theta=0.6$ and $q=0$. All other parameters are the same as in figure 7.8 . 
ities. The accuracy of this model may be limited by absence of factors such as geographic location, as mentioned above. It does however, with a bit of modification to some parameter values, allow us to model a stable rate of acute rheumatic fever, at values similar to that seen in the real data. Even without the manipulation of the acute rheumatic fever rates, the model produced a relatively stable, if overestimated, value for acute rheumatic fever numbers in New Zealand. It may be that with further development of the model and the inclusion of seasonality, socioeconomic status and geographic location, in some combination, less modification of parameters would be required to match what is seen in the real data.

Varying the rates of treatment in our model produced some interesting results. It has been suggested that primary prevention of acute rheumatic fever be targeted at high-risk individuals, rather than just the whole population. This has been suggested as a possibly efficient means of managing the ethnic inequality [18]. Figure 7.9 shows what happened when we tried treating Group A streptococcus, in individuals at high-risk of developing acute rheumatic fever, exclusively in our model. The results produced here suggest that focusing treatment to the exclusion of other groups could make acute rheumatic fever numbers worse. A higher rate of treatment for higher risk groups did result in lower acute rheumatic fever numbers however. As long as each risk group received some treatment for Group A streptococcus infections, focusing more attention on those at higher risk of developing acute rheumatic fever, produced desirable results.

It is possible that the increase in acute rheumatic fever numbers with exclusive treatment, may be due to the assumption of homogeneous mixing made by our model. An individual with a high risk of developing acute rheumatic fever, it is assumed is just as likely to catch Group A streptococcus from some one with a low risk of acute rheumatic fever risk as they are from someone with a very high risk. This even possibility of infection is not necessarily true to reality. Risk factors such as overcrowding show strong correlations with socioeconomic status and ethnicity. Ethnicity in turn is strongly correlated with geographic location. Factors such as preferential mixing within ethnicity neighbourhood and social standing may influence the impact the spread of Group A streptococcus and the impact of treatment protocols in the population. The development of acute rheumatic fever 
may be influenced by geographic location household structure as well. Before this type of model may be useful in management of acute rheumatic fever the inclusion of socio-economic and geographic factors, and the use of different mixing models must be investigated. Such further investigation is beyond the scope of this thesis While primary prevention of acute rheumatic fever in New Zealand is currently considered inadequate [18, this model is limited in how much we can learn with regard to treatment protocols. From what we have seen infection between groups may be an important factor and exclusive treatment would be unwise. Different interaction and mixing patterns between sub-populations are something that should be considered before a large treatment and/or prevention plan is initiated. As the ethnic gap in rheumatic fever rate continues to grow [18], the prevention of this disease becomes more and more important. This is a solvable problem that deserves more attention. 


\section{Appendix A}

\section{Matlab Code}

\section{A.1 SIR and SIS Stochastic Plots}

This is the code used to produce the DTMC sample paths in Sections 2.5.1 and 2.5.2. They each produce three sample paths where change in the number of infectious individuals at each step is determined by the value of a random number. Euler's method is used to to produce a plot of the deterministic model for comparison.

This is the code used to produce the SIS sample paths shown in Figure 2.5.

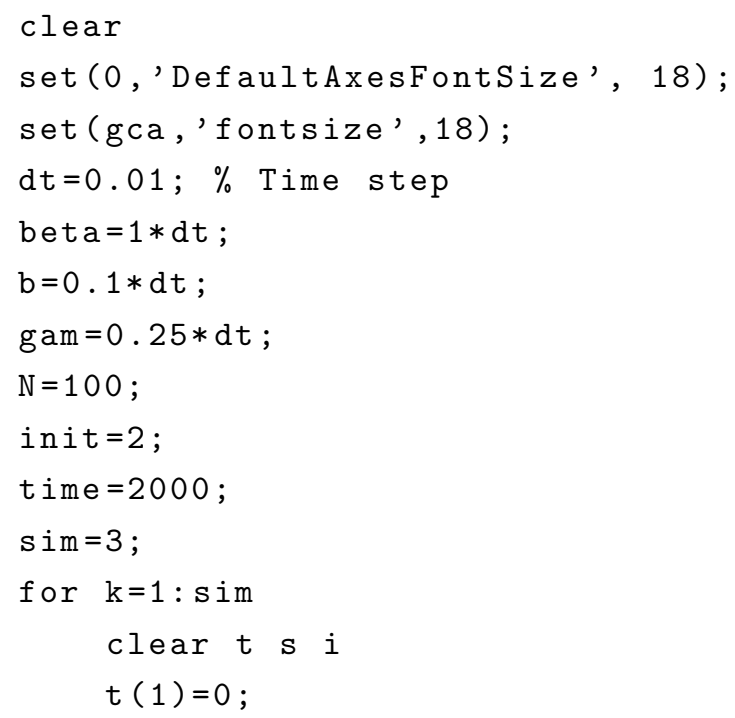




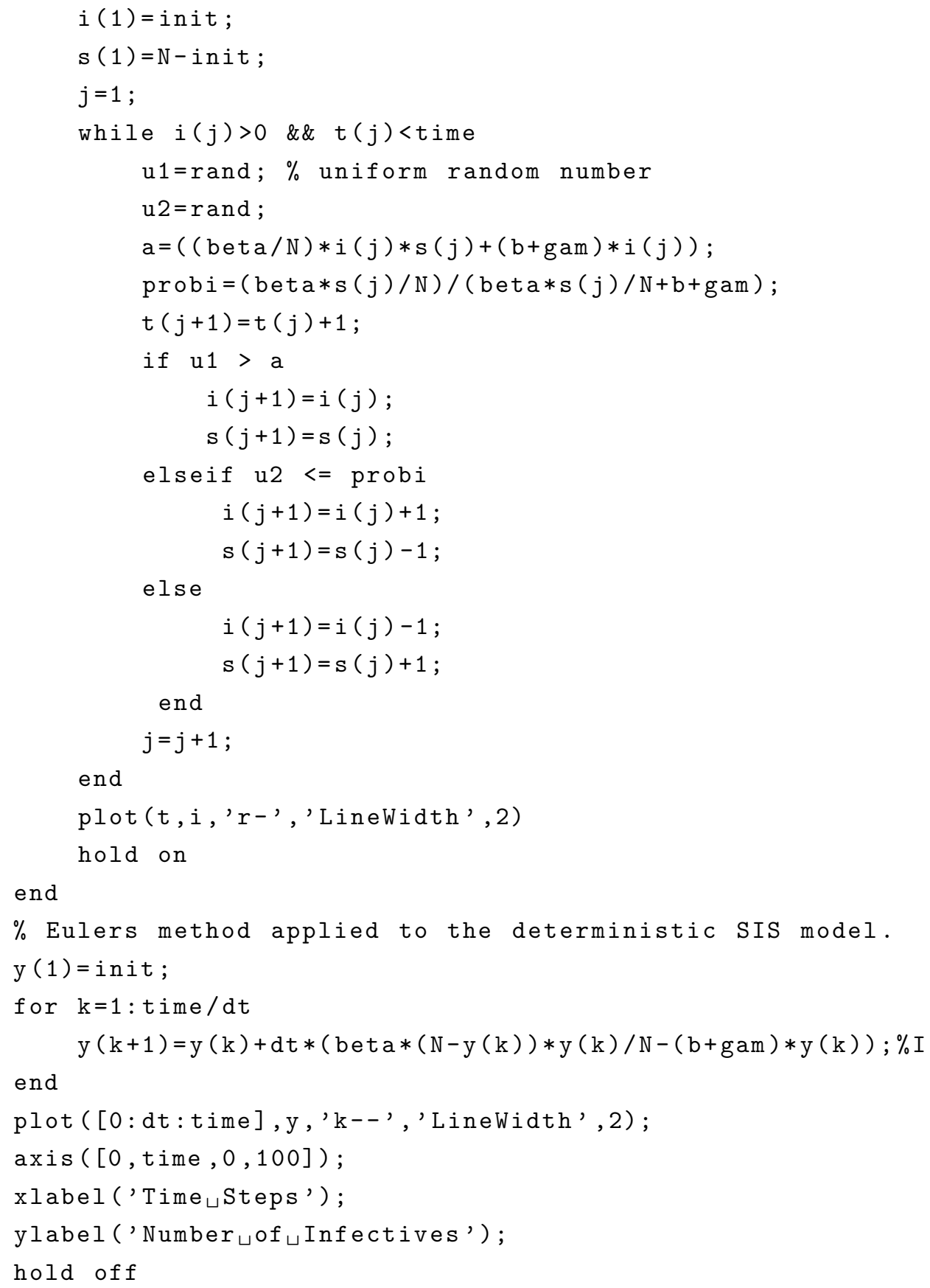

Below is the code used to produce the SIR DTMC sample paths shown in Figure 2.6.

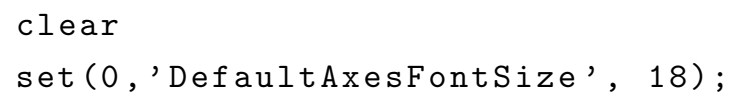




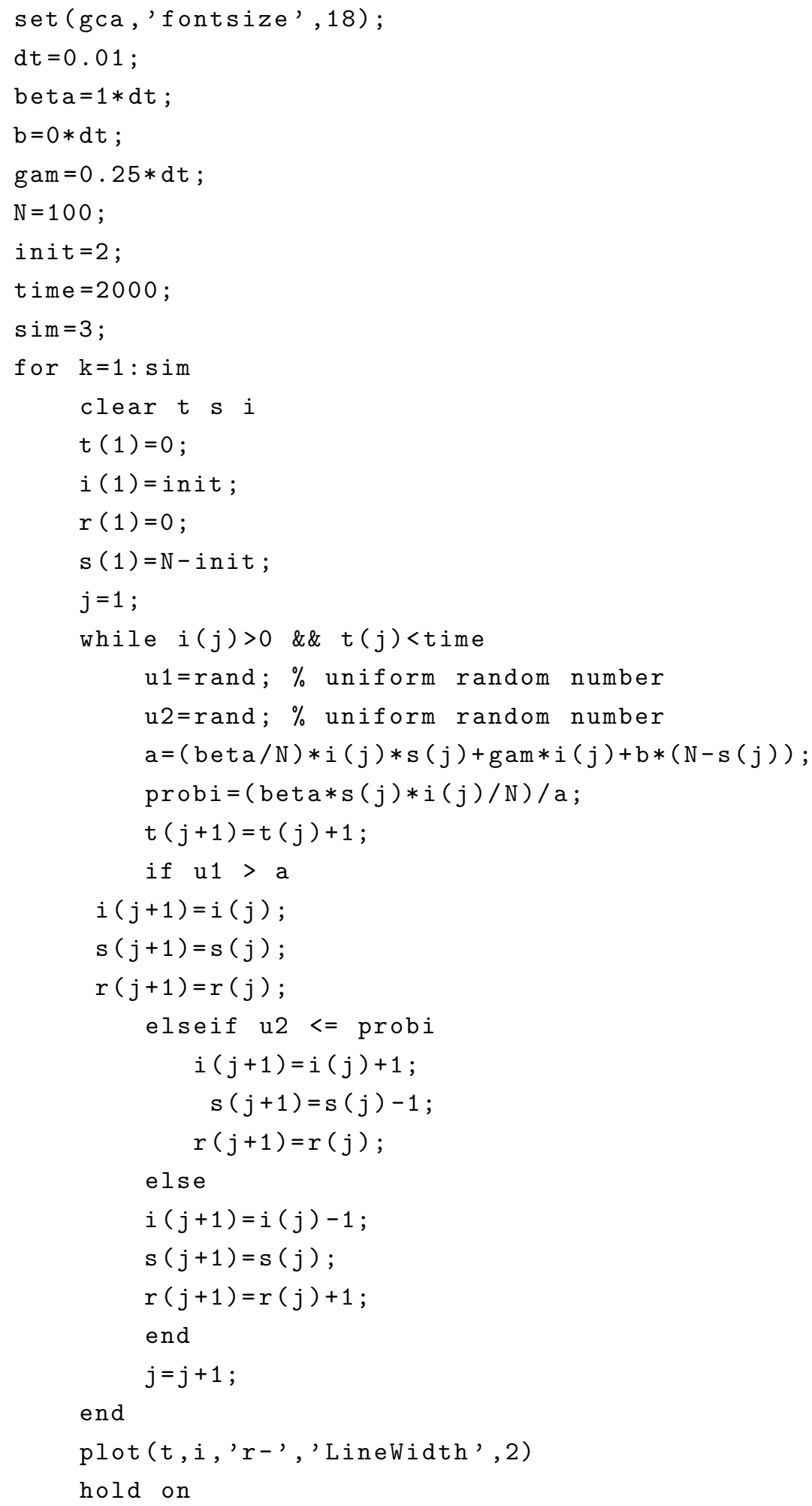


end

$\%$ Euler's method applied to the deterministic SIR model.

$\mathrm{y}(1)=$ init $; \% \mathrm{y}=\mathrm{i}$

$\mathrm{x}(1)=0 ; \quad \% \mathrm{x}=\mathrm{r}$

for $k=1: t i m e / d t$

$\mathrm{x}(\mathrm{k}+1)=\mathrm{x}(\mathrm{k})+\mathrm{dt} *(\mathrm{gam} * \mathrm{y}(\mathrm{k})-\mathrm{b} * \mathrm{x}(\mathrm{k})) ; \% \mathrm{R}$

$\mathrm{y}(\mathrm{k}+1)=\mathrm{y}(\mathrm{k})+\mathrm{dt} *((\operatorname{beta} * \mathrm{y}(\mathrm{k}) *(\mathrm{~N}-(\mathrm{x}(\mathrm{k})+\mathrm{y}(\mathrm{k})))) / \mathrm{N}-(\mathrm{b}+\mathrm{gam}) * \mathrm{y}(\mathrm{k})) ; \% \mathrm{I}$

end

plot ([0:dt:time],y, 'k--', 'LineWidth', 2);

axis $([0$, time , 0,60]);

xlabel ('Time $\sqcup$ Steps');

ylabel ('Number $\sqcup$ of $\sqcup$ Infectives');

hold off

The code below was used to produce the three sample paths shown in figure 2.7 . The time it takes for a change of stare to occur is determined by $t(j+1)=$ $t(j)-\log (u 1) / a$. where $u 1$ is a random number.

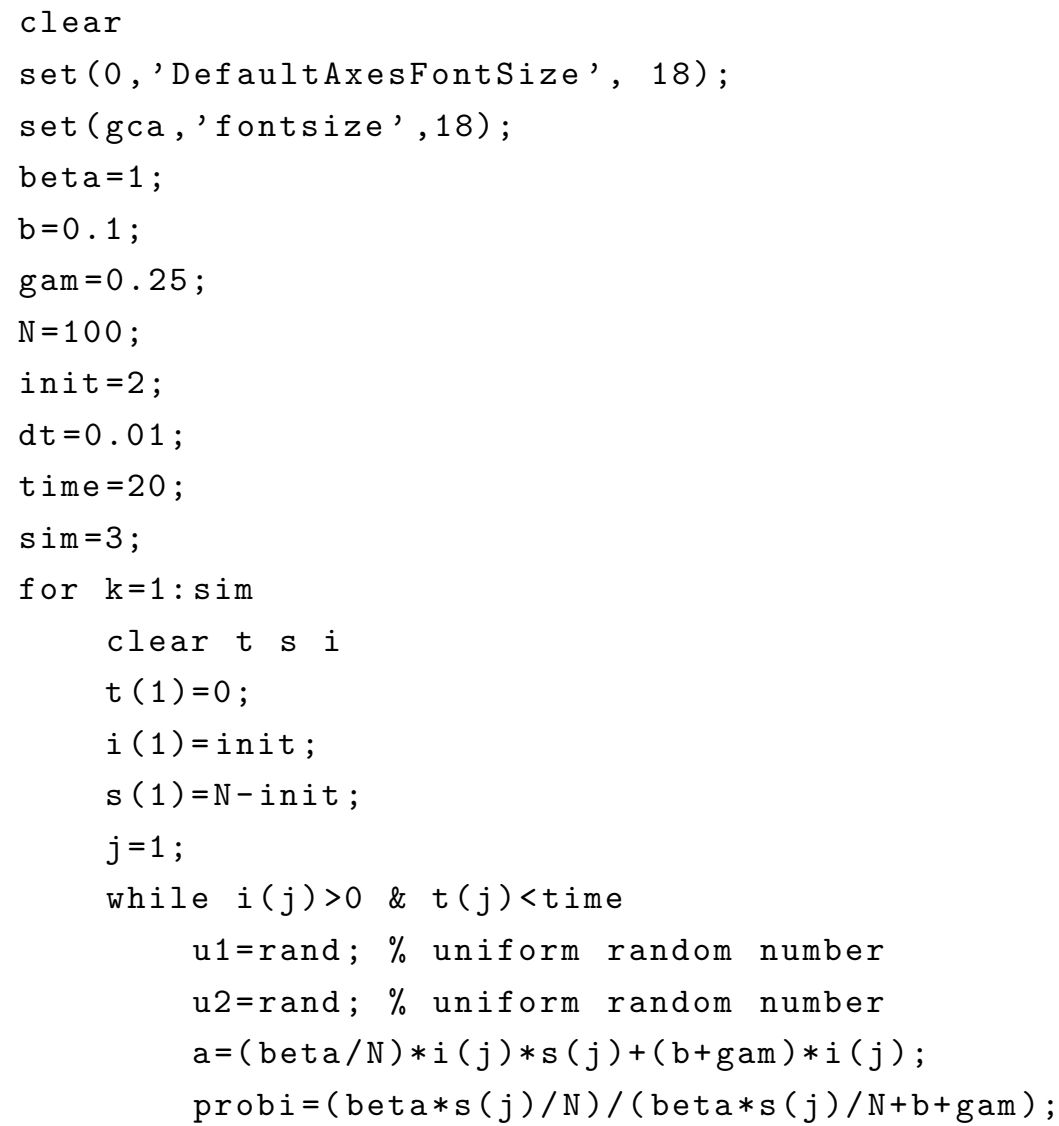




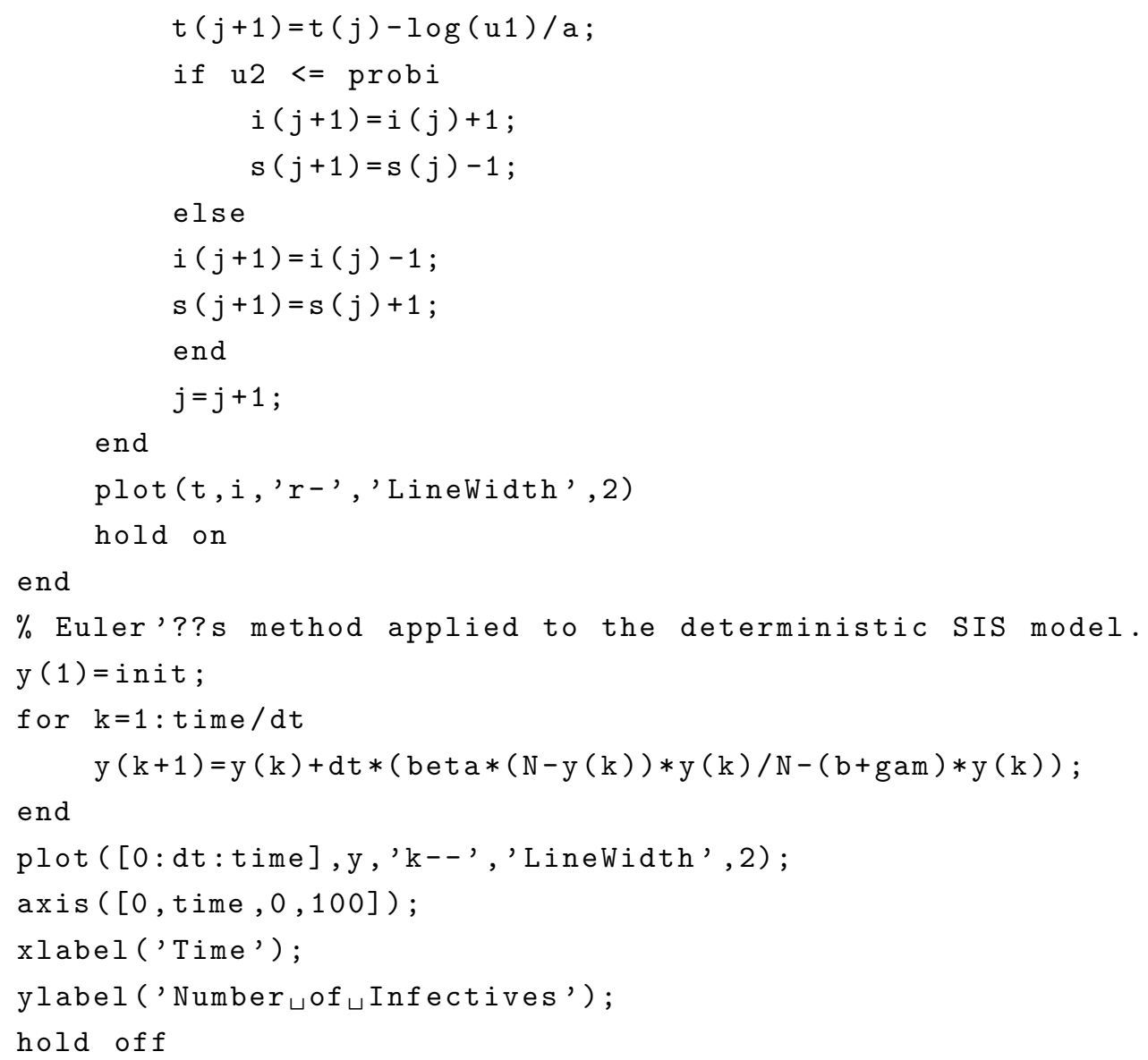

\section{A.2 SIAS Plots}

This section contains the code used to produce the deterministic and stochastic plots seen in Chapter 4

\section{A.2.1 Deterministic Plot}

The bit of code below defines the function we are trying to solve.

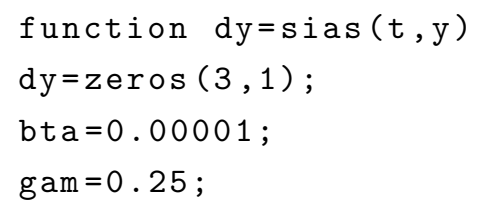




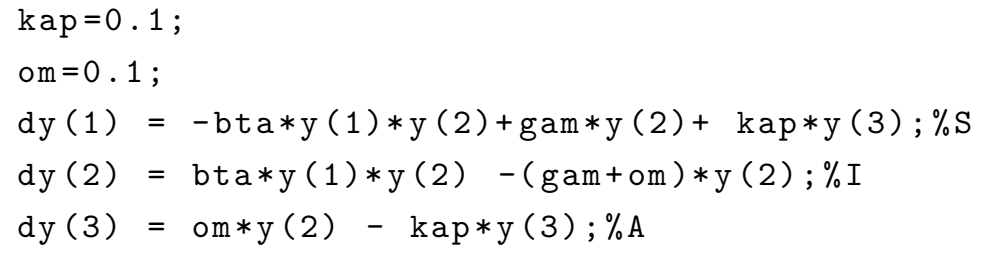

This next bit of code uses the Runge-Kutta method to solve the system, given parameter and initial values. It then produces a plot of the solution, as seen in Figure 4.2 .

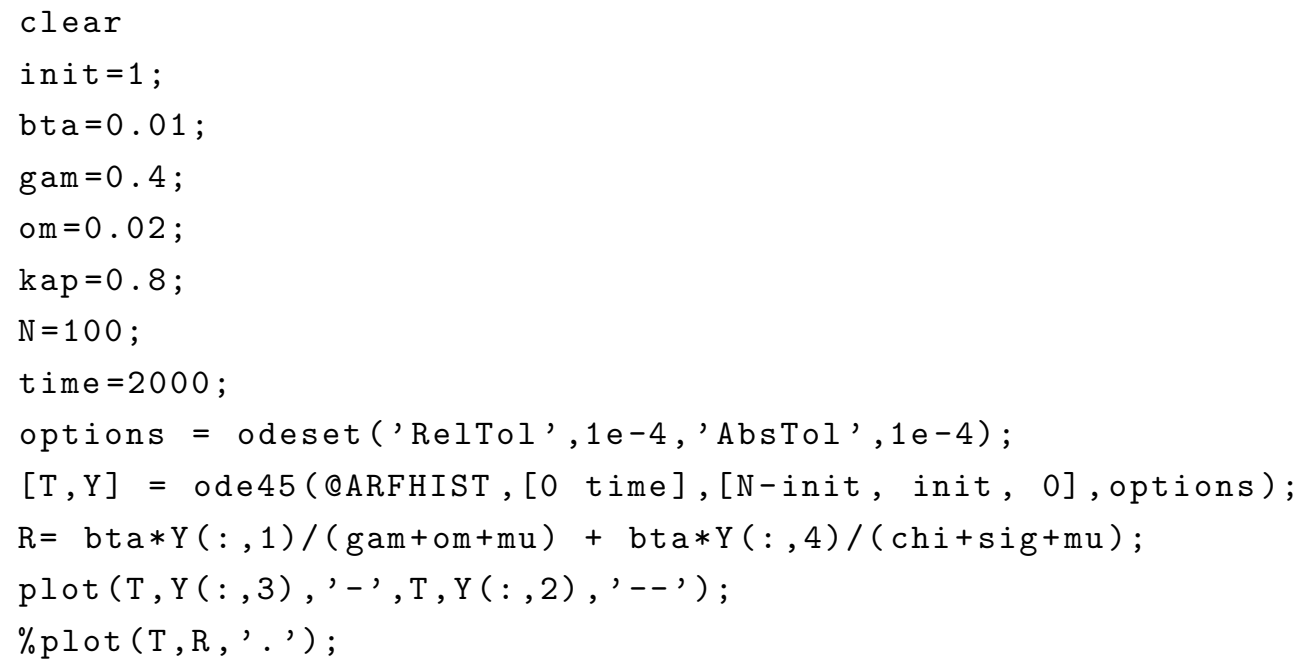

\section{A.2.2 Stochastic Plots}

This is the code used to produce the stochastic plots for the basic SIAS model. Both programmes produce three sample paths and plot them along with the deterministic model.

The code used to produce the DTMC sample paths in the Figure 4.5 is shown below.

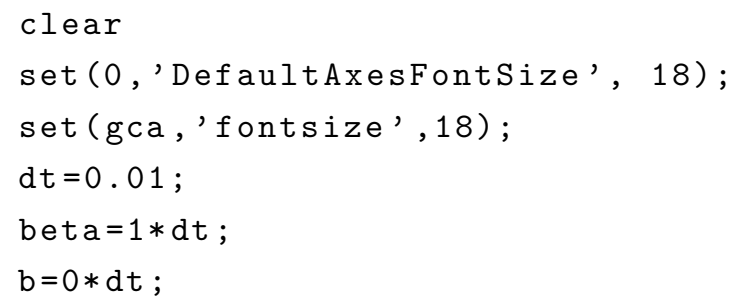




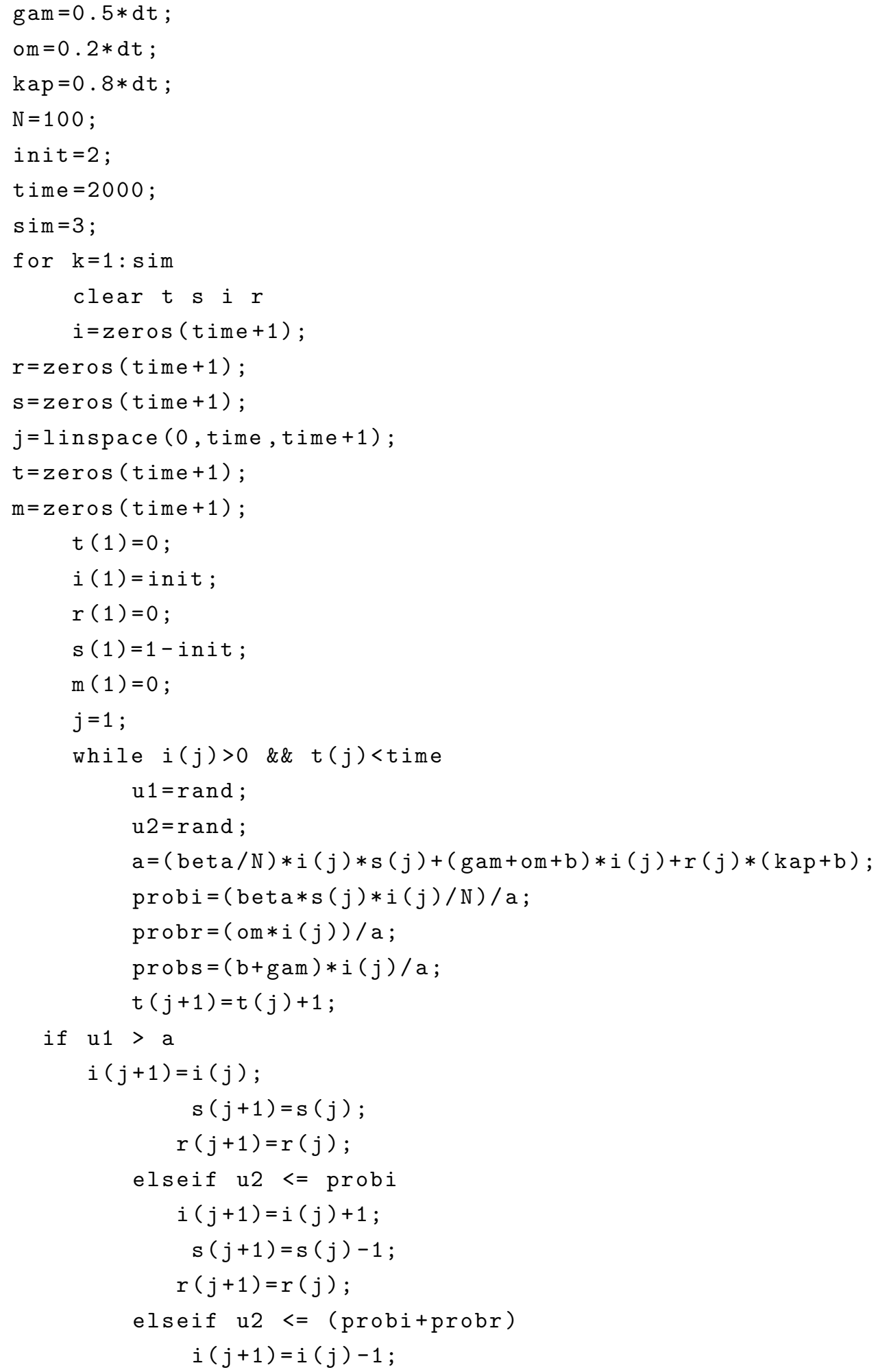




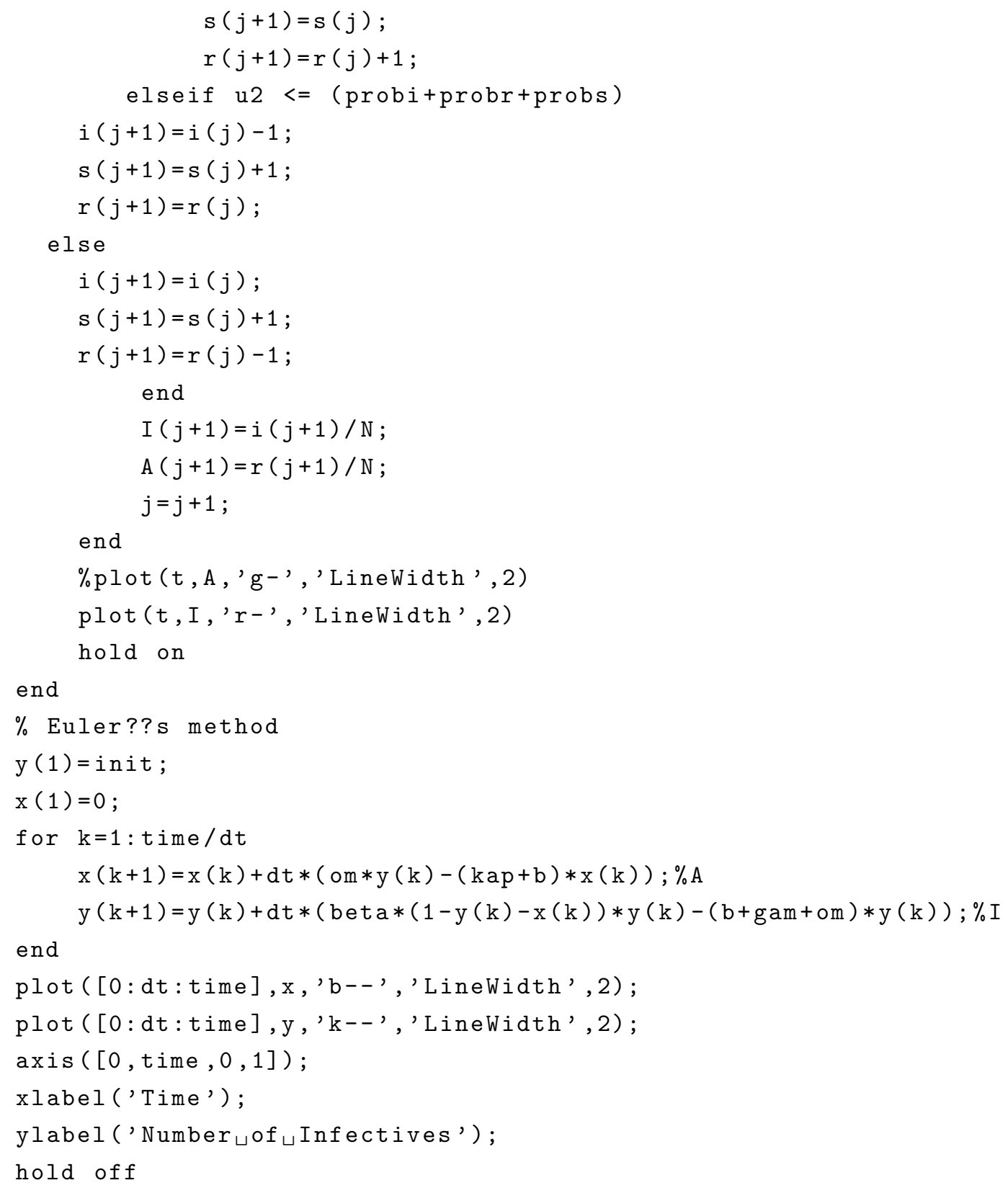

The CTMC sample paths seen in Figure 4.6 were produced using the following code.

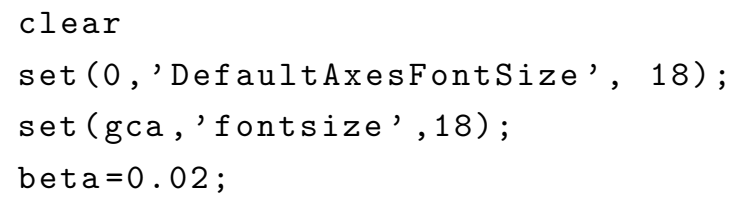




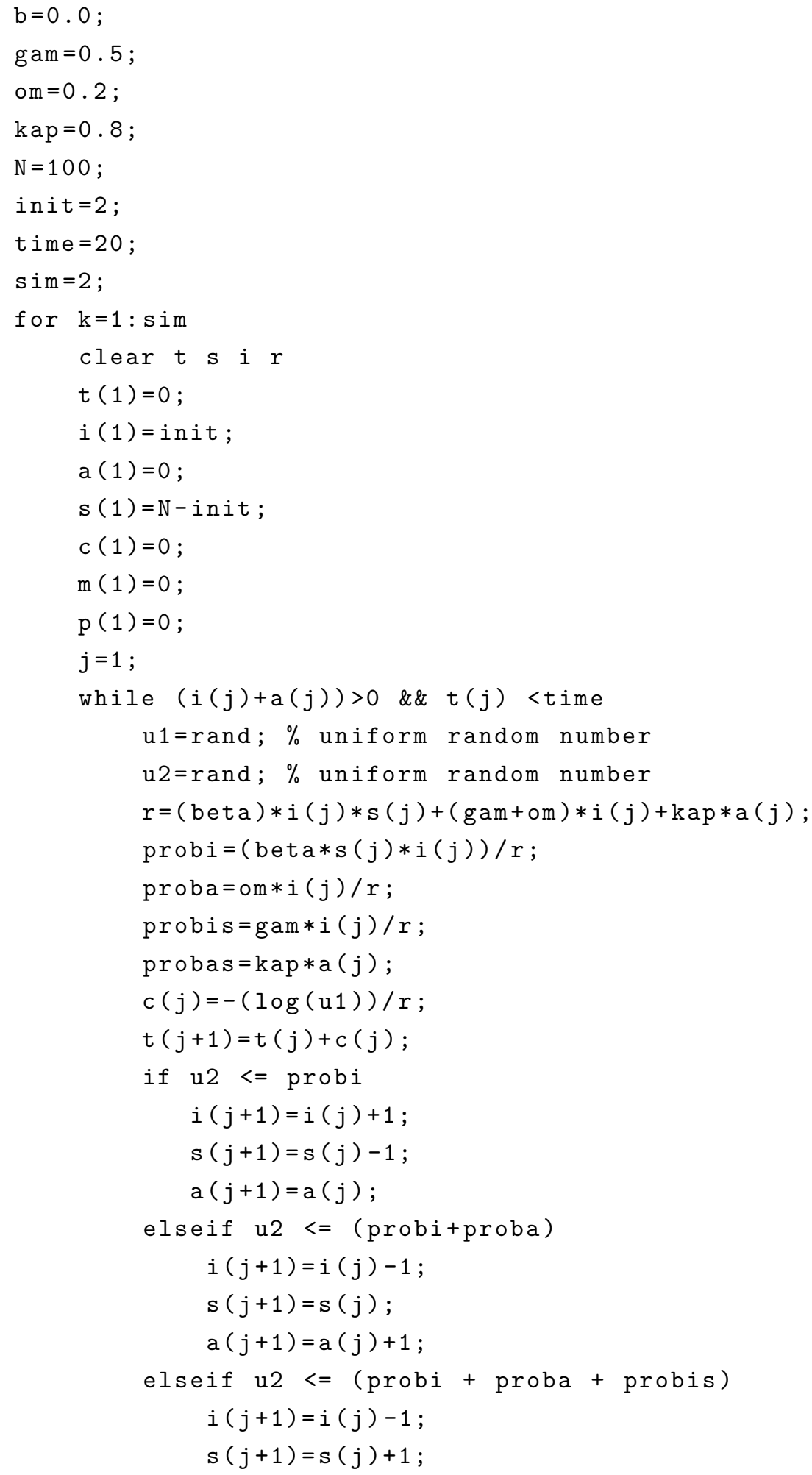




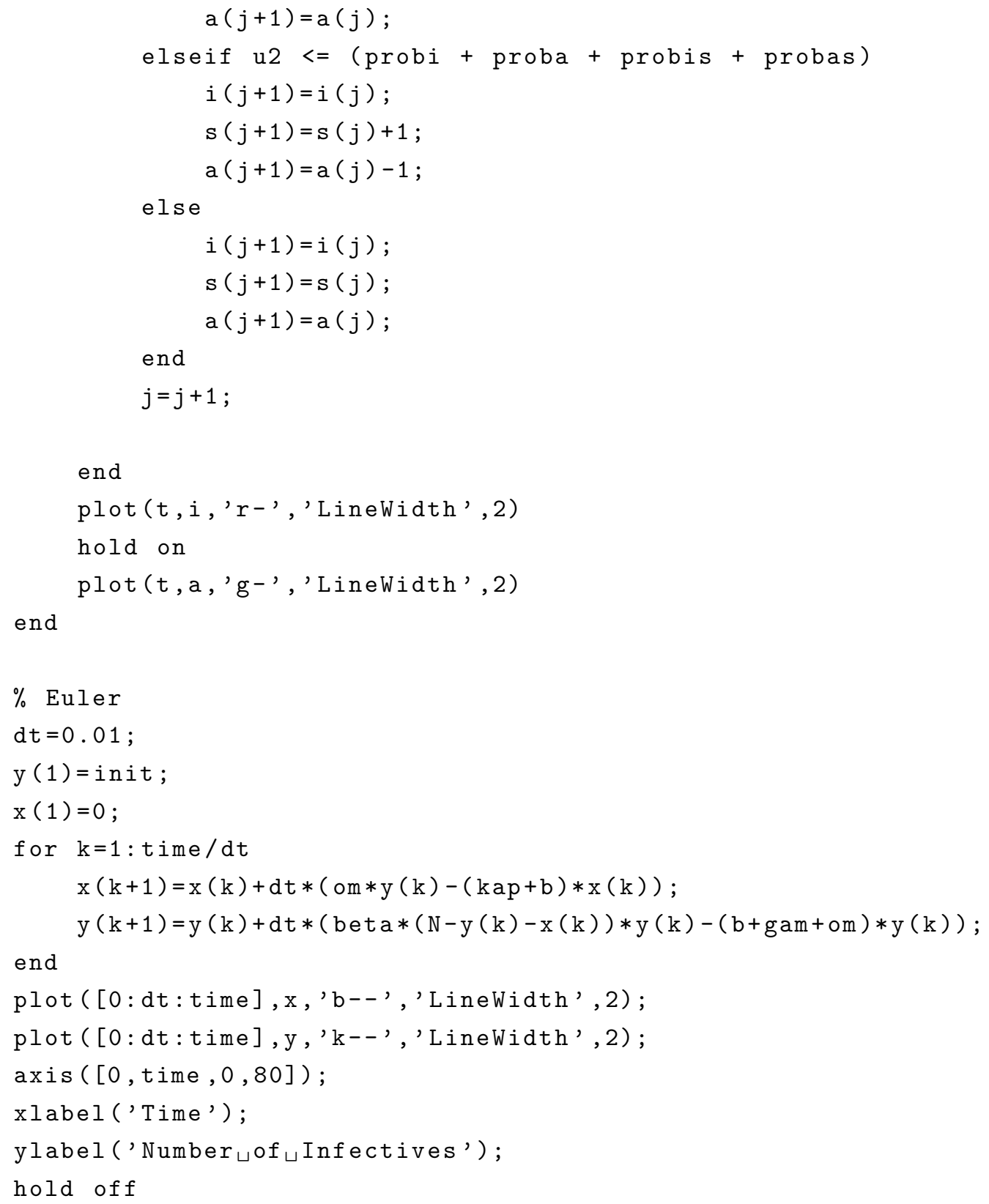

\section{A.2.3 SIAS with Vital Dynamics}

This is the code that was used to produce the two sample paths seen in Figure 5.2. In this case we have used ode45 and the Runge-Kutta method to plot the deterministic model. 


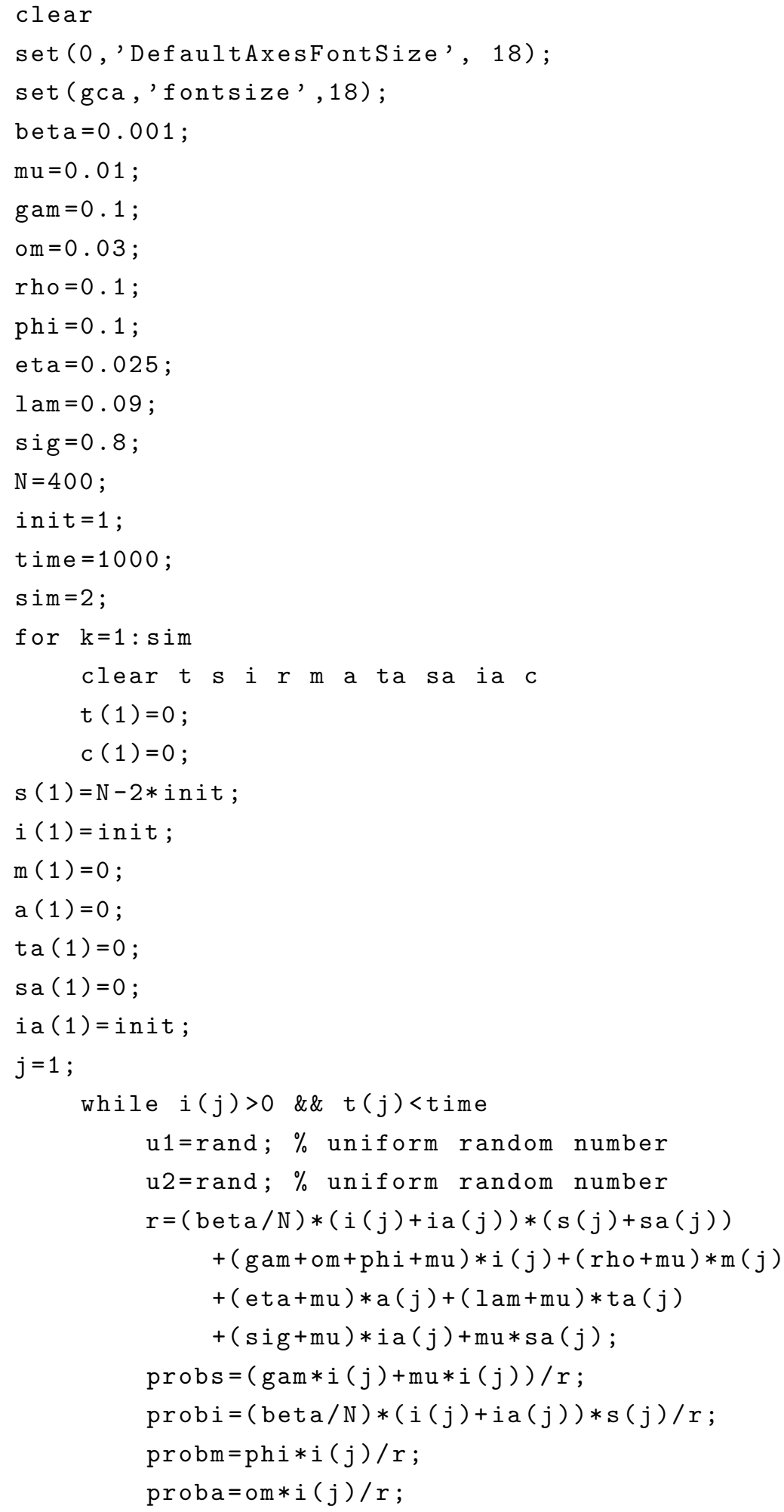




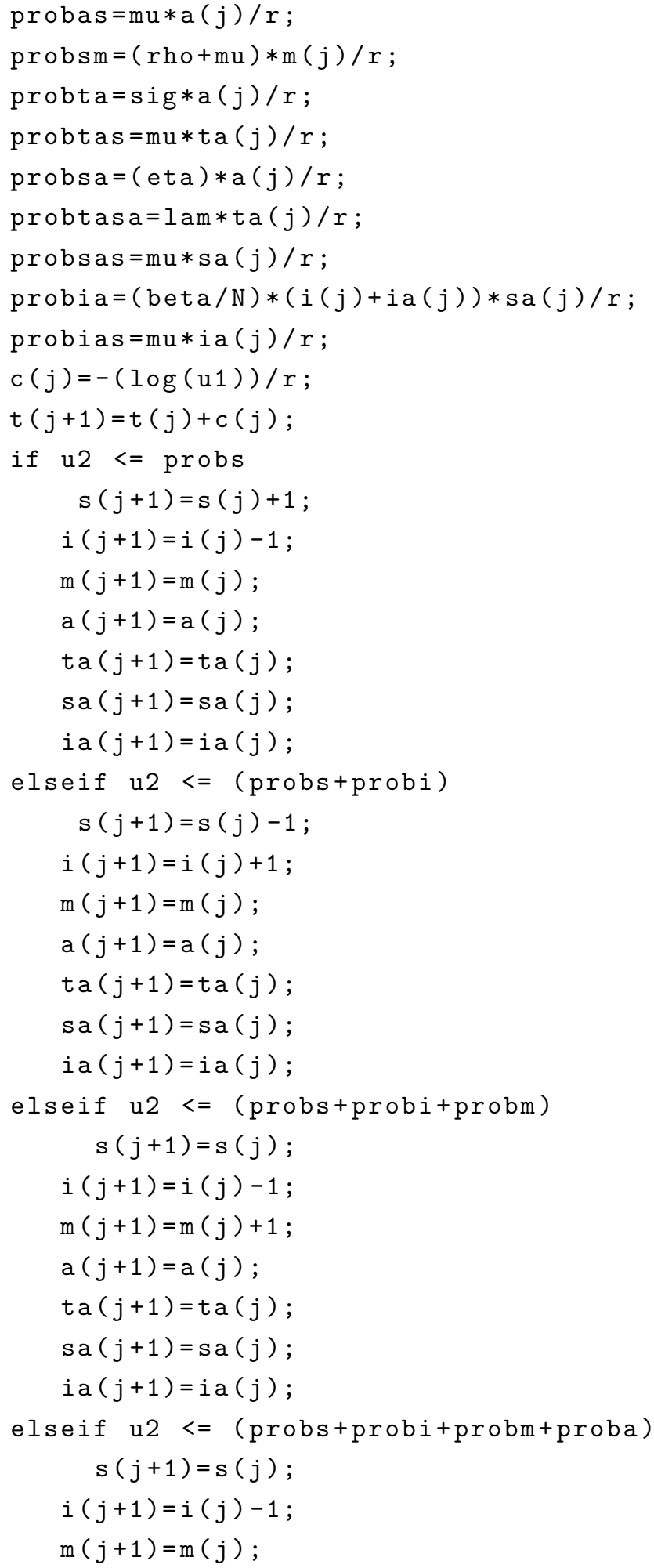




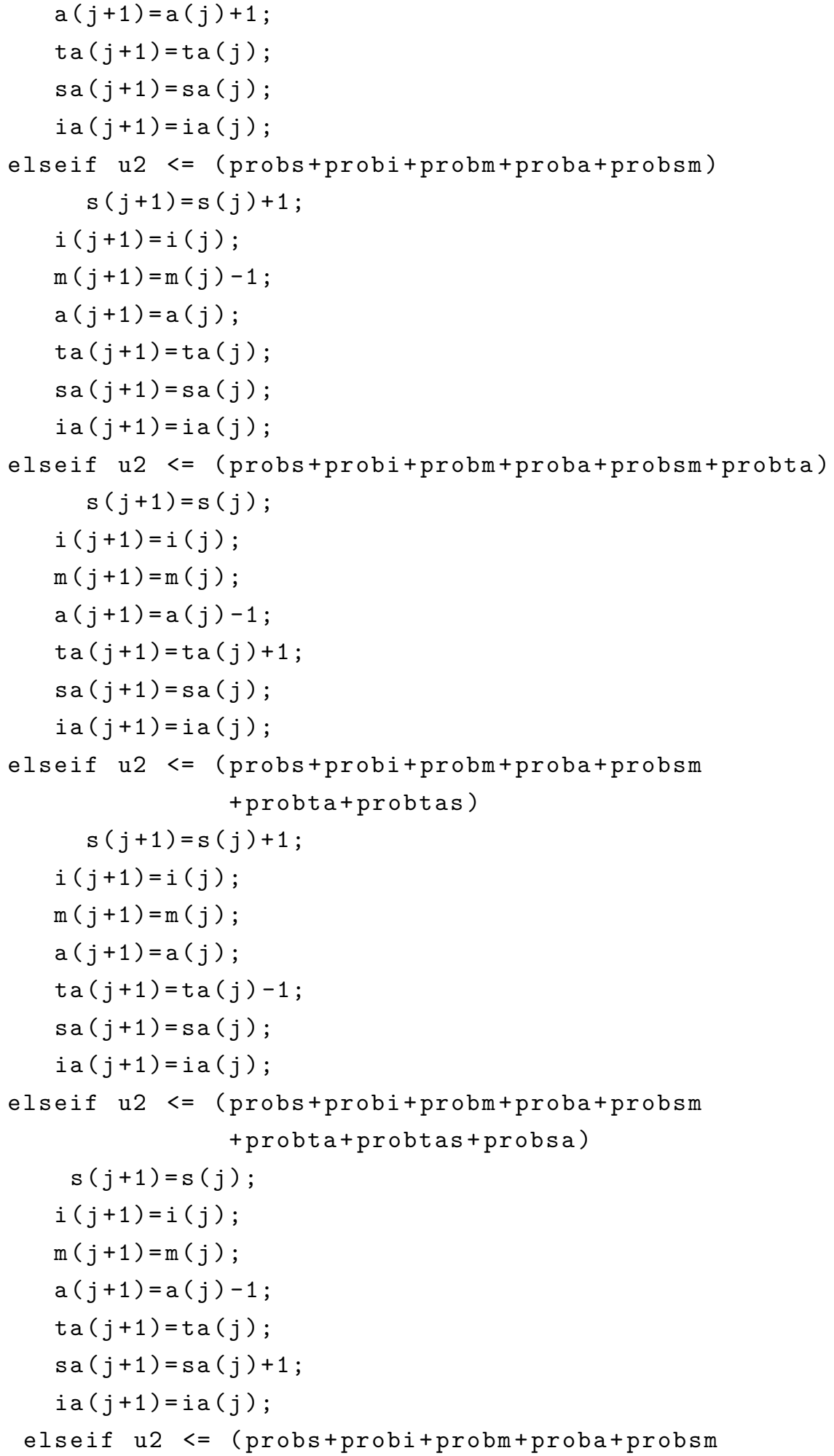




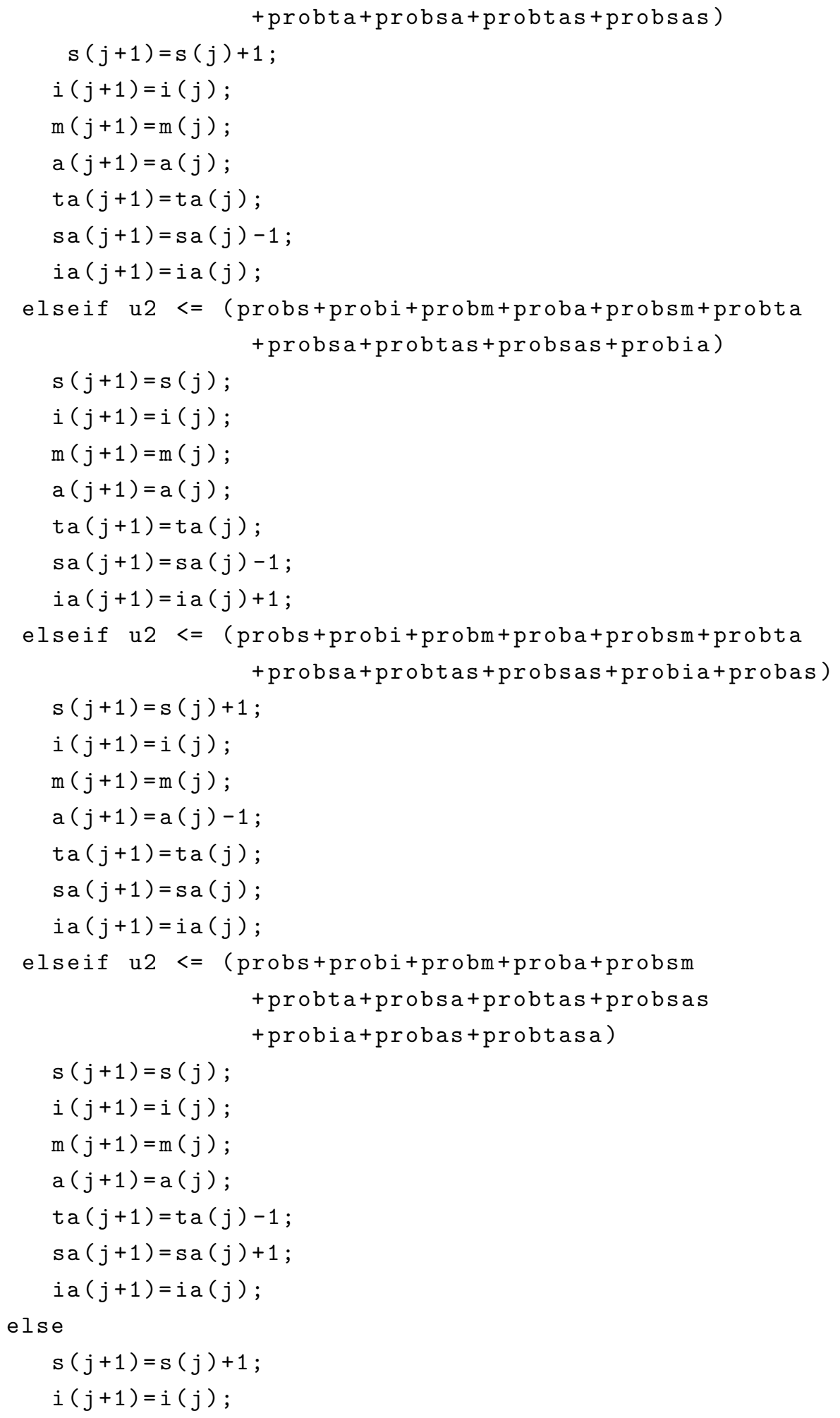




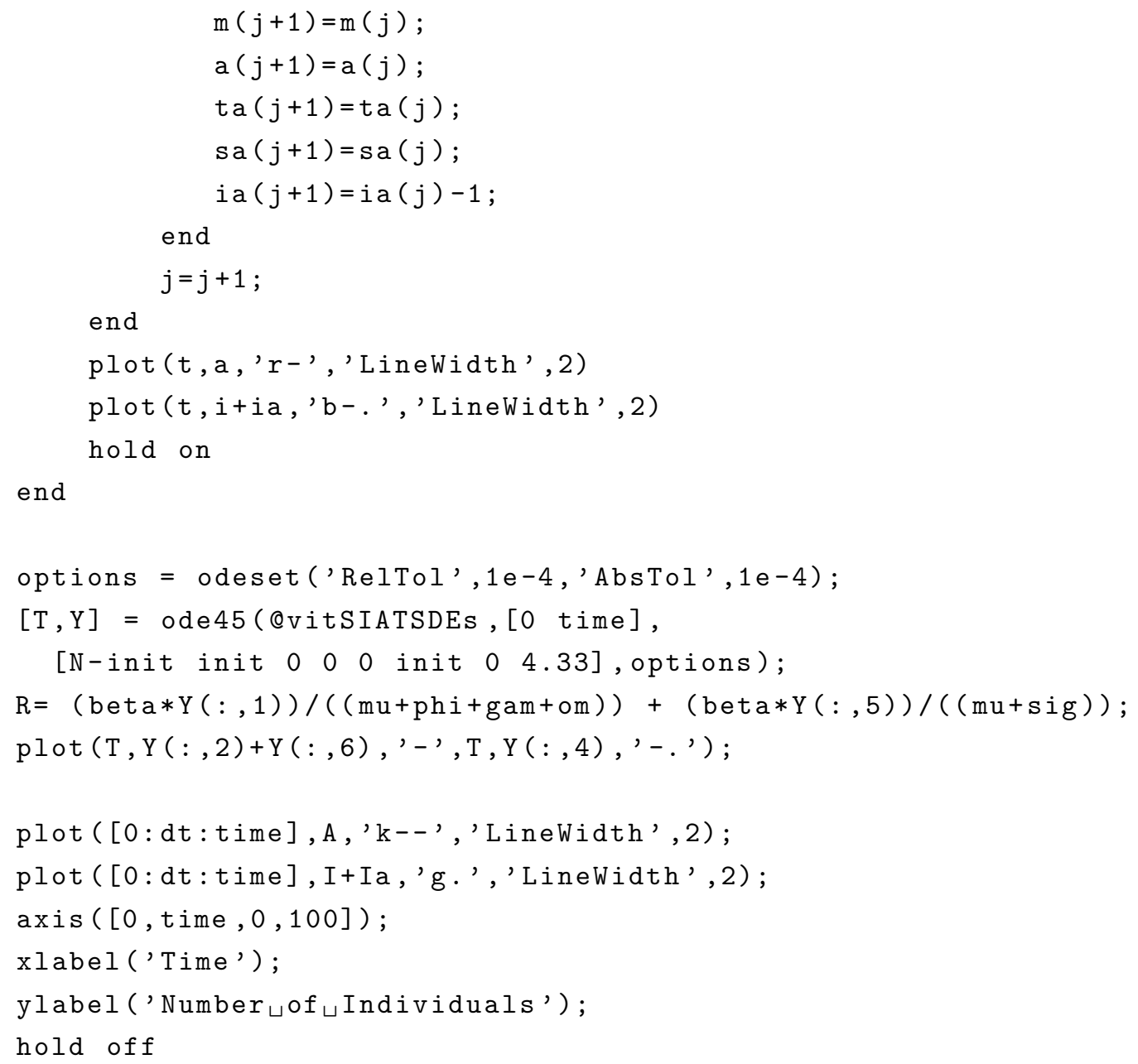

The code used to input the deterministic model for solving is given below.

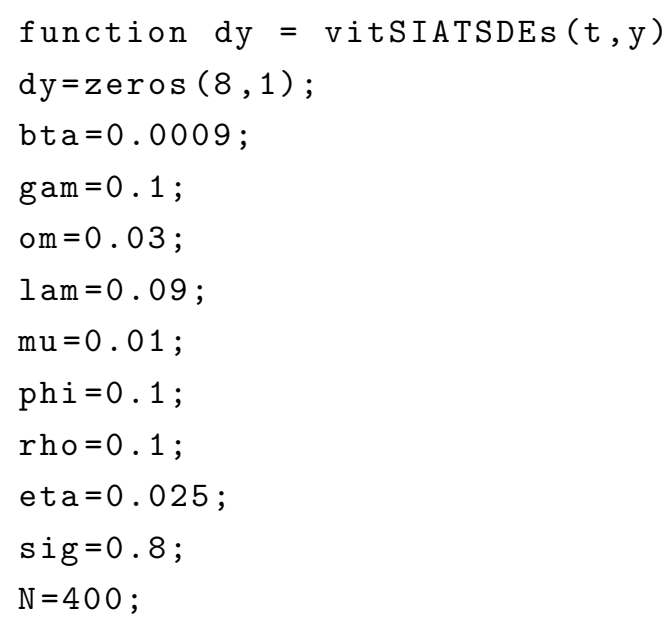




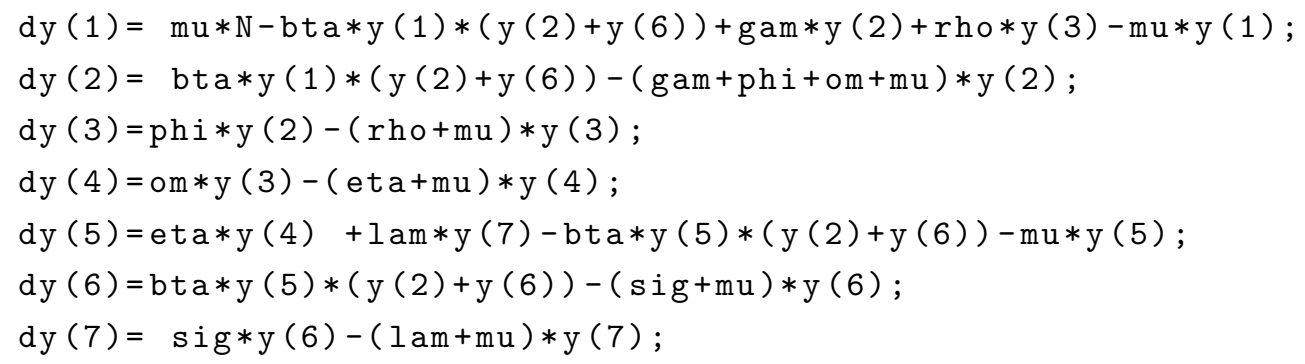

\section{A.3 New Zealand Specific Model}

This is the code that was used to produce the plots in Chapter 7 . The values of the parameters were varied from plot to plot. ode45 uses the Runge-Kutta to solve the function labeled MPIage, defined below. $\mathrm{R}$ was used to plot the reproduction number.

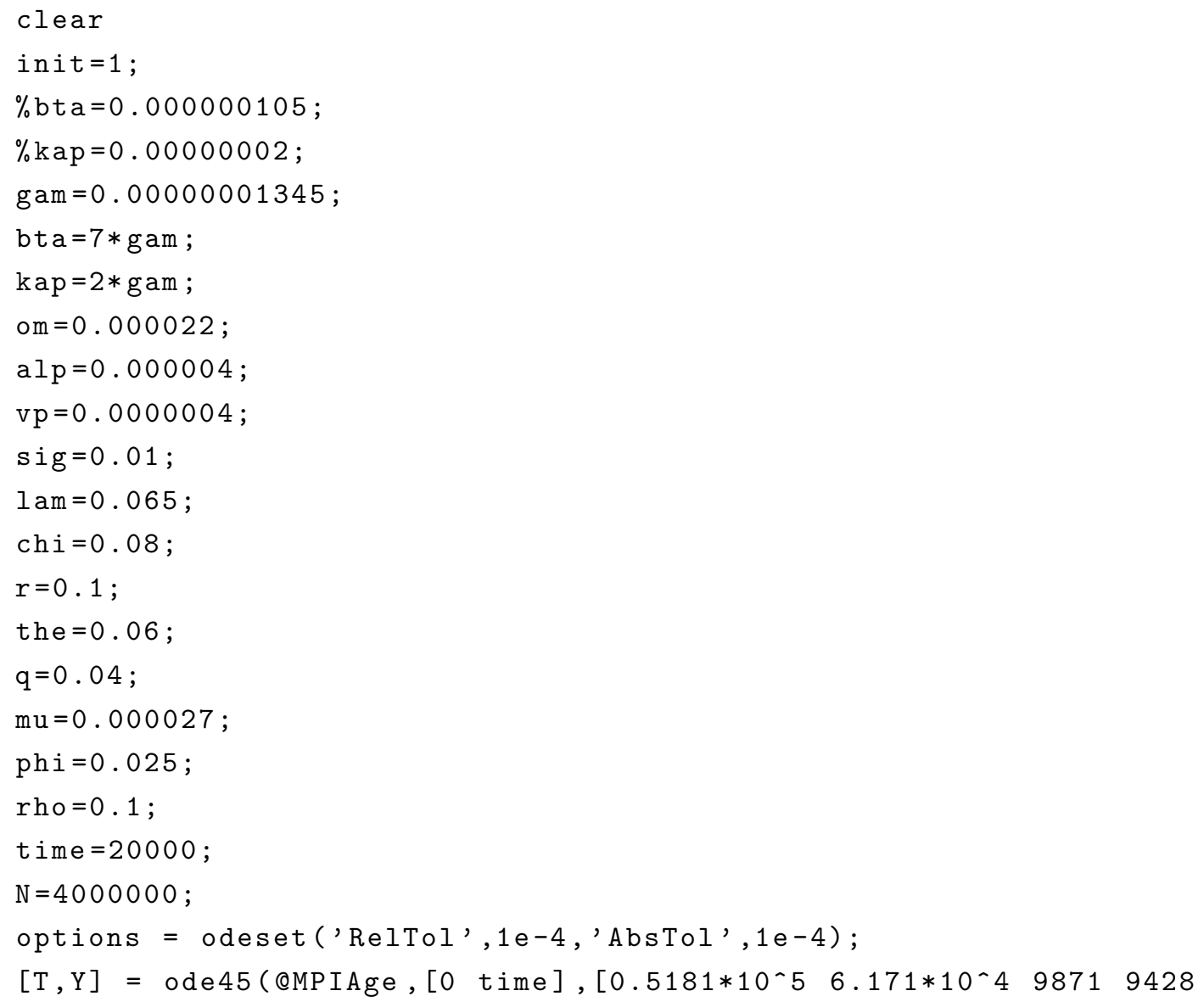




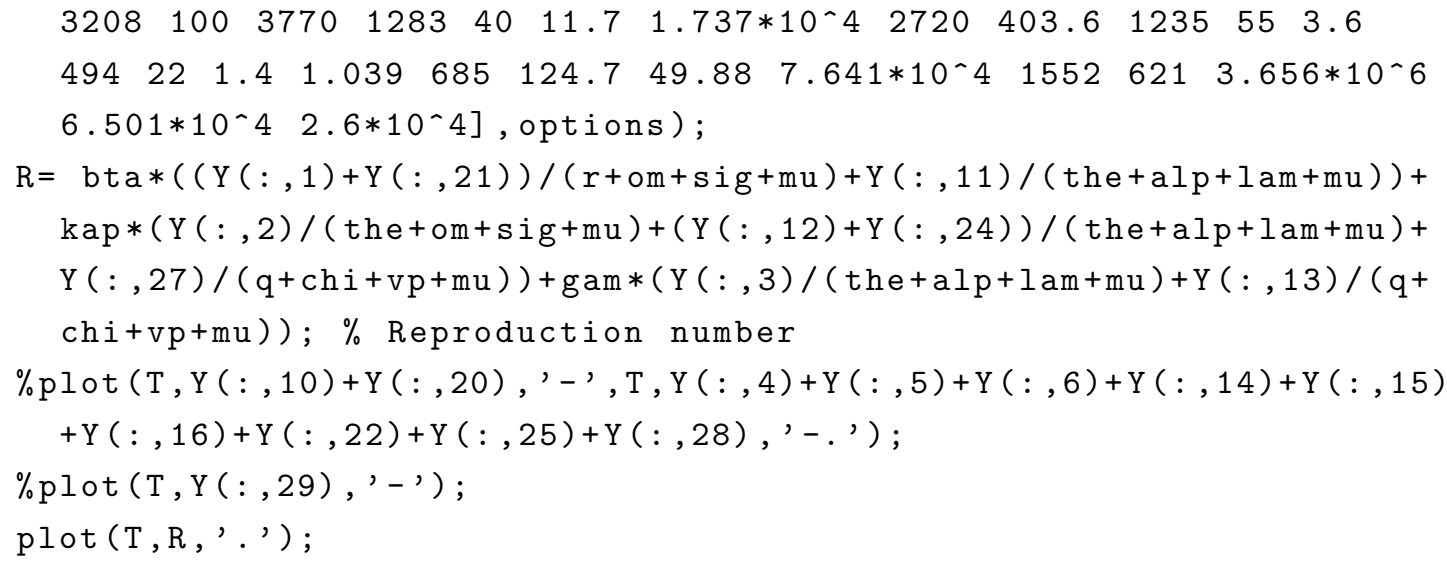

The code below was defines the model we are wanting to plot

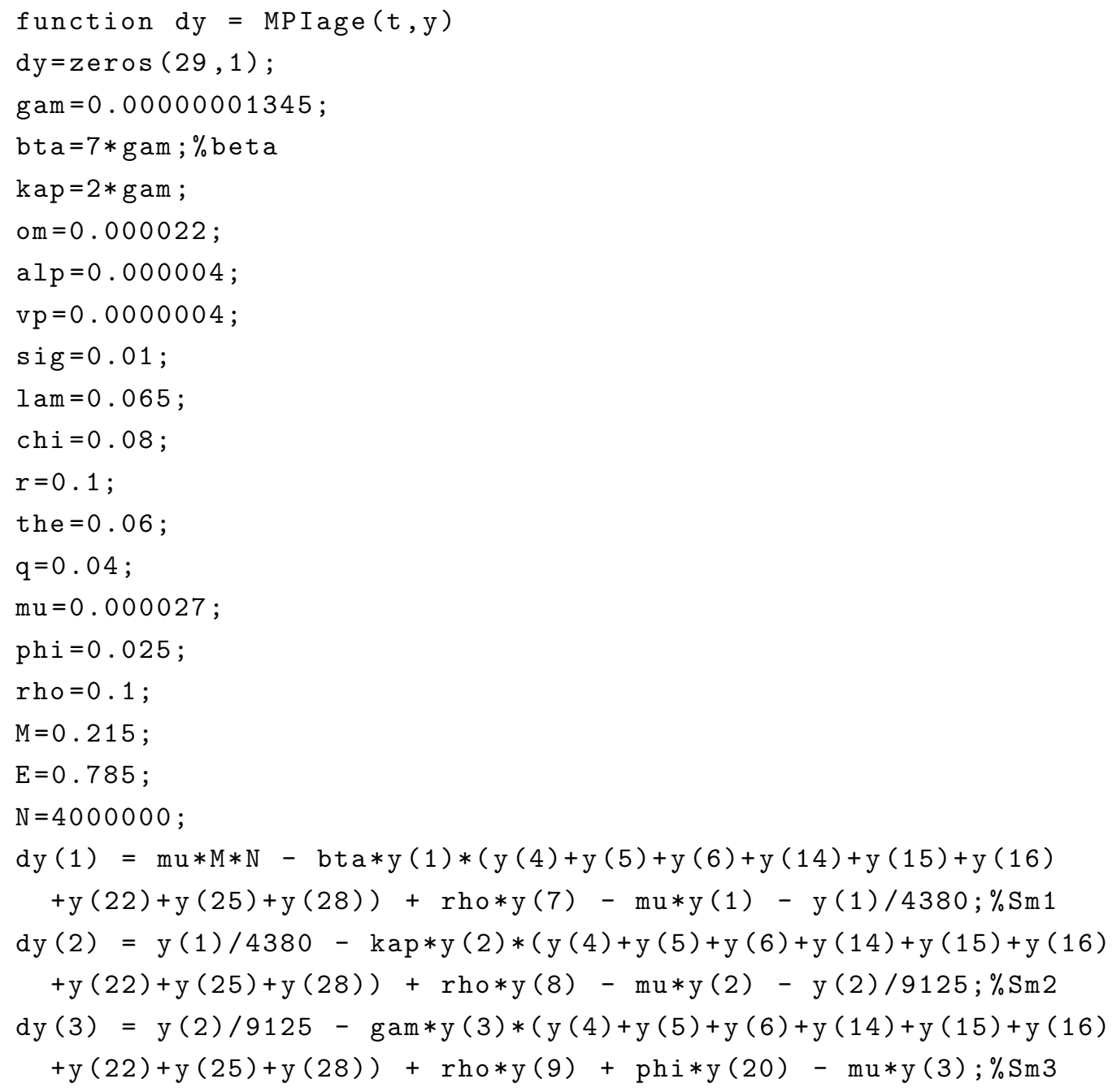




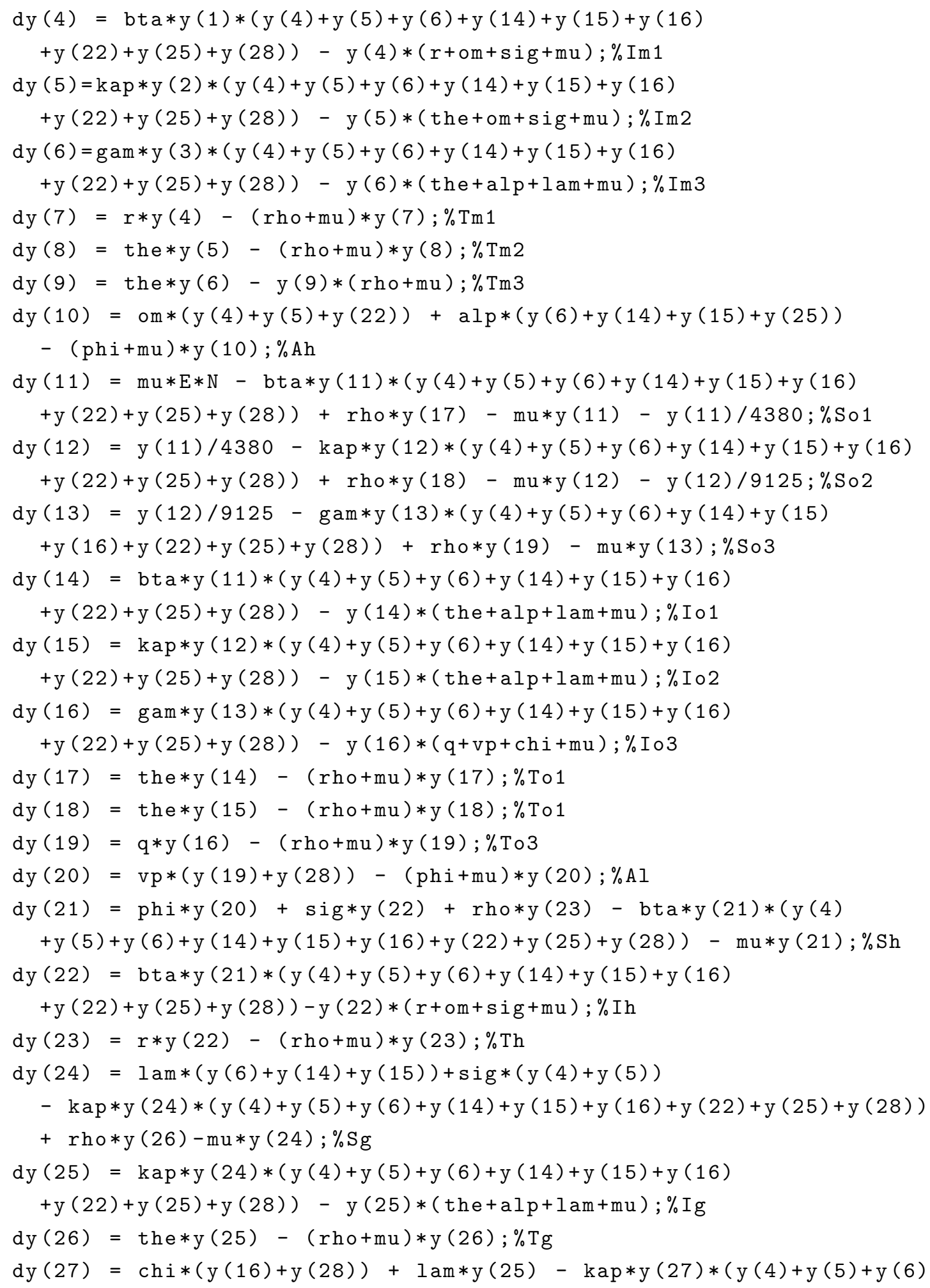


$+\mathrm{y}(14)+\mathrm{y}(15)+\mathrm{y}(16)+\mathrm{y}(22)+\mathrm{y}(25)+\mathrm{y}(28))+\mathrm{rho} * \mathrm{y}(29)-\mathrm{mu} * \mathrm{y}(27) ; \% \mathrm{SI}$

$\mathrm{dy}(28)=\mathrm{kap} * \mathrm{y}(27) *(\mathrm{y}(4)+\mathrm{y}(5)+\mathrm{y}(6)+\mathrm{y}(14)+\mathrm{y}(15)+\mathrm{y}(16)$

$+\mathrm{y}(22)+\mathrm{y}(25)+\mathrm{y}(28))-\mathrm{y}(28) *(\mathrm{chi}+\mathrm{q}+\mathrm{vp}+\mathrm{mu}) ; \% I I$

$\mathrm{dy}(29)=\mathrm{q} * \mathrm{y}(28)-(\mathrm{rho}+\mathrm{mu}) * \mathrm{y}(29) ; \% \mathrm{Tl}$ 


\section{Bibliography}

[1] Ayoub, E., And Ahmed, S. Update on complications of group a streptococcal infections. Current problems in pediatrics 27, 3 (1997), 90.

[2] Bascand, G. Births and Deaths: Year ended March 2012. Statistics New Zealand. http://is.gd/qigegu.

[3] Bisno, A., Gerber, M., Gwaltney, J., Kaplan, E., and Schwartz, R. Practice guidelines for the diagnosis and management of group a streptococcal pharyngitis. Clinical Infectious Diseases 35, 2 (2002), 113.

[4] Bisno, A., Gerber, M., JaCk, M., Et Al. Diagnosis and management of group a streptococcal pharyngitis: a practice guideline. Clinical Infectious Diseases 25, 3 (1997), 574.

[5] Boccara, N. Modeling Complex Systems, 2nd ed. Springer, 2010.

[6] Brauer, F. Simple models of invasions and epidemics. 2009 Summer School on The Mathematics of Invasions and Epidemiology.

[7] Brauer, F., Van den Driessche, P., And Wu, J. Lecture Notes in Mathematical Epidemiology. Springer, 2008.

[8] Bryant, P., Robins-Browne, R., Carapetis, J., and Curtis, N. Some of the people, some of the time: susceptibility to acute rheumatic fever. Circulation 119, 5 (2009), 742.

[9] Carapetis, J., Steer, A., Mulholland, E., and Weber, M. The global burden of group a streptococcal diseases. The Lancet infectious diseases 5, 11 (2005), 685-694. 
[10] Chin, T., Chin, E., Siddiqui, T., And Sundell, A. Rheumatic heart disease. Online)(Cited 2006 May 19). Available from URL: http://. emedicine. medscape. com/article/891897-overview (2010).

[11] Cilliers, A. Rheumatic fever and its management. BMJ: British Medical Journal 333, 7579 (2006), 1153.

[12] Cunningham, M. Pathogenesis of group a streptococcal infections. Clinical microbiology reviews 13, 3 (2000), 470-511.

[13] Diekmann, O., And Heesterbeek, J. Mathematical epidemiology of infectious diseases: model building, analysis, and interpretation, vol. 5. Wiley, 2000.

[14] Diekmann, O., Heesterbeek, J., and Metz, J. On the definition and the computation of the basic reproduction ratio $\mathrm{r} 0$ in models for infectious diseases in heterogeneous populations. Journal of mathematical biology 28, 4 (1990), 365-382.

[15] Gockenbach, M. Partial differential equations: analytical and numerical methods. Society for Industrial \& Applied Mathematics, 2011.

[16] Graunt, J. Natural and Political Observations made upon the Bills of Mortality. The Johns Hopkins Press, 1939.

[17] Hale, J., And KoÇAK, H. Dynamics and bifurcations, vol. 1 of Texts in Applied Mathematics. Springer-Verlag New York, 1991.

[18] Jaine, R., Baker, M., And Venugopal, K. Epidemiology of acute rheumatic fever in new zealand 1996-2005. Journal of paediatrics and child health 44, 10 (2008), 564-571.

[19] Kasper, D., Braunwald, E., Fauci, A., Hauser, S., Longo, D., JAMESON, J., AND ISSELBACHER, K. Harrison's principles of internal medicine, 18 ed. McGraw-Hill, 2005.

[20] Keeling, M., Gilligan, C., Et Al. Metapopulation dynamics of bubonic plague. Nature 40\%, 6806 (2000), 903-906. 
[21] Member of Rheumatic Fever Guidelines Writing Group. Group A Streptococcal Sore Throat Management, May 2008.

[22] Ministry of Health, New Zealand. Health Act (1956).

[23] New Zealand Public Health Observatory. Notifiable Disease. http://www.nzpho.org.nz/NotifiableDisease.aspx.

[24] Olivier, C. Rheumatic feveris it still a problem? Journal of Antimicrobial Chemotherapy 45, suppl 1 (2000), 13-21.

[25] O'Sullivan, L., ET Al. Rheumatic Fever in Māori: What can we do better? Best Practice 37 (August 2011), 22-33.

[26] Perko, L. Differential equations and dynamical systems, vol. 7 of Texts in Applied Mathematics. Springer, 2000.

[27] Rammelkamp, C., Wannamaker, L., and Denny, F. The epidemiology and prevention of rheumatic fever. 1952. Bulletin of the New York Academy of Medicine 74, 1 (1997), 119.

[28] Roberts, M. G. Modeling strategies for containing an invading infection. Mathematical Population Studies 13, 4 (2006), 205-214.

[29] Somro, A., And Rehman, R. Pharyngitis and sore throat: A review. African Journal of Biotechnology 10, 33 (2011), 6190-6197.

[30] Stein, W., ET AL. Sage Mathematics Software (Version 4.7.1). The Sage Development Team, 2011. http://www . sagemath.org.

[31] Stollerman, G. Rheumatic fever in the 21st century. Clinical infectious diseases 33, 6 (2001), 806.

[32] Stollerman, G., Lewis, A., Schultz, I., and Taranta, A. Relationship of immune response to group a streptococci to the course of acute, chronic and recurrent rheumatic fever. The American journal of medicine 20, 2 (1956), 163-169. 
[33] WigGins, S. Introduction to applied nonlinear dynamical systems and chaos, vol. 2. Springer, 2003. 